\title{
A RADIO-PROPAGATION-MEASUREMENT-BASED STUDY OF THE CAPACITY OF MULTI-USER DISTRIBUTED-MIMO INDOOR CHANNELS AT 18 AND 28 GHZ
}

\author{
by
}

\author{
MOHAMAD ALKADAMANI
}

\author{
A thesis submitted to the \\ Faculty of Graduate and Postdoctoral Affairs \\ in conformity with the requirements for
}

the degree of Master of Applied Science in Electrical and Computer Engineering

\author{
Carleton University \\ Ottawa, Ontario, Canada
}

September 2019

Copyright (c) mohamad alkadamani, 2019 


\begin{abstract}
This thesis had the objective of determining whether indoor SHF wireless systems can provide the same or greater system capacities as current $2 \mathrm{GHz}$ systems. In the interest of avoiding obstruction losses, the focus was on the use of directive antennas at frequencies above $2 \mathrm{GHz}$ to receive radio signals from reflectors and scatterers that could be stronger than those received over direct Tx-to-Rx antenna paths. Models for indirect path transmission loss, spatial variations, shadowing, and temporal fading were derived from propagation measurements conducted in the 2-30 GHz bands.

As a final step, models derived from the propagation measurements were used in multi-user conventional and distributed MIMO system capacity simulations. It was shown that with currently achievable transmit powers and antenna gains and beamwidths, narrow band systems at 18 and $28 \mathrm{GHz}$ can be designed to offer the same or greater capacity as those operating in the $2 \mathrm{GHz}$ band.
\end{abstract}




\section{Acknowledgements}

First and foremost, I want to thank my co-supervisors Dr. Robert Bultitude and Professor Ian Marsland. I am thankful to Dr. Bultitude for the encouragement and the endless support throughout my master studies. His advice on both research as well as on my career have been invaluable. Working with him was an honour and a lifetime experience. I would like to appreciate Professor Marsland for the enormous contribution of time and ideas to increase the productivity of my thesis. I would also like to thank my committee members Prof. Roshdy Hafez, Prof. Ramy Gohary, and Prof. Claude D'Amours for their invaluable comments and feedback about many aspects of my thesis.

I would like to acknowledge Ericsson Canada, the Natural Sciences and Engineering Research Council of Canada (NSERC), and Mitacs Canada for the financial support of this work. I especially would like to thank Ron Casselman, Dr. Gary Boudreau, Dr. Georgy Levin, and Neil McGowan of Ericsson Canada for their helpful discussions and support.

I am deeply grateful to my parents for their never-ending care, incomparable upbringing, and support of my academic aspirations. I also offer sincere thank to my uncle Hicham and my parents-in-law. I would like to exhibit my sincere gratitude to my brothers Yaser and Ghiath, my sister Mais, and their families. My deepest 
gratitude is reserved for my lovingly wife, Nour. Without her tolerance, patience and unfailing support, completing this thesis would not have been possible. Last but not least, I would like to dedicate this work to my beloved son, Jude, without his smiles, I would have never been able to continue. 


\section{Contents}

Abstract $\quad$ i

Acknowledgements $\quad$ ii

Contents $\quad$ iv

List of Tables $\quad$ vii

List of Figures viii

List of Acronyms xiv

Chapter 1: Introduction 1

1.1 Thesis Background . . . . . . . . . . . . . . . . . . . 1

1.2 Thesis Motivation . . . . . . . . . . . . . . . . . . 2

1.3 Thesis Contributions . . . . . . . . . . . . . . . . 3

1.4 Literature Review . . . . . . . . . . . . . . . . . . . . . 5

1.4.1 Radio Propagation and Channel Measurements . . . . . . . . 5

1.4.2 Small Scale Fading . . . . . . . . . . . . . . . . . . . . . . . . 10

1.4.3 Distributed-MIMO Systems . . . . . . . . . . . . . . 11

1.5 Thesis Organization . . . . . . . . . . . . . . . . . 13

Chapter 2: Physical Phenomena of Particular Interest in Considering Indoor Wireless Communications at Frequencies Between $6 \mathrm{GHz}$ and $30 \mathrm{GHz} \quad 14$

2.1 Large-Scale Transmission Loss . . . . . . . . . . . . . . . . . . . . . 15

2.1.1 Free Space Transmission . . . . . . . . . . . . . . . . . 15

2.2 Diffraction Loss . . . . . . . . . . . . . . . . . . . . . 19

2.2.1 Knife-Edge Diffraction . . . . . . . . . . . . . . . . . . . 19

2.2.2 Fresnel Zones . . . . . . . . . . . . . . . . . . . . . 25

2.3 Multipath Propagation . . . . . . . . . . . . . . . . . . . 29

2.3.1 Two-Ray Model . . . . . . . . . . . . . . . . . . 29 
2.4 Small Scale Fading and Multipath . . . . . . . . . . . . . . . 31

Chapter 3: $\quad$ Propagation Measurement System 32

3.1 Phase 1: Wideband Radio Channel Measurement System . . . . . . . 32

3.1.1 Wideband Radio Channel Measurement Techniques . . . . . . 34

3.1 .2 Measurement Setup . . . . . . . . . . . . . . . . . . . 37

3.1.3 Mitigation of Multipath Interference _. . . . . . . . . . . 45

3.1.4 Noise Floor of the VNA Sounding System . . . . . . . . . . 48

3.1.5 Receive Antenna Directivity and Control of Receive Antenna Pointing Direction . . . . . . . . . . . . . . . . . 49

3.2 Phase II: Single Tone Measurement System . . . . . . . . . . . . . . 51

3.2 .1 CW Transmitter . . . . . . . . . . . . . . . . 52

3.2 .2 CW Receiver . . . . . . . . . . . . . . . . . . . 53

3.2 .3 Data Collection System . . . . . . . . . . . . . . . 54

Chapter 4: Propagation Measurements and Modelling for Indoor Channels $\quad 55$

4.1 Phenomenological Measurements _. . . . . . . . . . . . . 55

4.1.1 Empty Room Measurements . . . . . . . . . . . . . . 56

4.1.2 Diffraction Loss from One Obstruction . . . . . . . . . . 59

4.1.3 Measurement of Transmission via Indirect Paths Around a Single Obstruction . . . . . . . . . . . . . . . . 62

4.1.4 Measurement of Transmission via Opening Between Two Obstructions . . . . . . . . . . . . . . . . . 75

4.2 Propagation Measurements in Clutter and Channel Modelling . . . . 80

4.2.1 Transmission Loss and Shadowing . . . . . . . . . . . . . 83

$4.2 .2 \quad$ Static RMS Delay . . . . . . . . . . . . . . . . . . . 91

4.3 Characterization of Temporal Variations on Indoor Radio Channels . 95

4.3.1 Link Budget Analysis . . . . . . . . . . . . . . . . . . 95

4.3.2 Propagation Measurement Scenarios and Procedures _. . . . 97

4.4 Observations . . . . . . . . . . . . . . . . . . . . . . . . . . . . . . 98

4.5 Data Analysis . . . . . . . . . . . . . . . . . . . . . . . 98

4.5.1 Modelling of Envelope Fading Distribution . . . . . . . . . 104

\section{Chapter 5: On the Capacity of Multi-User Distributed MIMO Chan-} nels 106

5.1 System Model . . . . . . . . . . . . . . . . . . . . . . . . . . 108

5.1.1 Capacity of MIMO Channels . . . . . . . . . . . . . . 110

5.2 Simulation Process of Multi-User D-MIMO System Capacity . . . . . 112

5.3 Simulation Results . . . . . . . . . . . . . . . . . . . 117

5.3.1 Results for $18 \mathrm{GHz}$ band . . . . . . . . . . . . . . . . . 119 
5.3.2 Results for $28 \mathrm{GHz}$ band . . . . . . . . . . . . . . . . 121

$\begin{array}{ll}\text { Chapter 6: Summary and Conclusion } & 127\end{array}$

6.1 Future Work . . . . . . . . . . . . . . . . . . 130

$\begin{array}{ll}\text { Bibliography } & 132\end{array}$

Appendix A: Rayleigh and Ricean Distribution Functions 139

Appendix B: Method of Generating Rayleigh and Ricean Fading Envelopes 141 


\section{List of Tables}

3.1 Antenna Specifications . . . . . . . . . . . . . . . 50

4.1 Obstruction and indirect path losses in the case of a single obstruction and bare walls . . . . . . . . . . . . . . . . . . 68

4.2 Obstruction and indirect path losses in case of a single obstruction and a computer monitor placed against the right side wall . . . . . . . 70

4.3 RMS delay spreads . . . . . . . . . . . . . . . . . . . . . . . . . . 74

4.4 Transmission loss incurred by propagation through an opening between two bookcases . . . . . . . . . . . . . . . . . . . . . 78

4.5 Delay dispersion values for the case of a $15 \mathrm{~cm}$ aperture between two bookcases ........................ . . 80

4.6 Statistics of the channel RMS Delay spreads for the three frequency

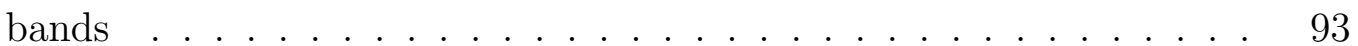

4.7 Link budget of CW equipment for different distances . . . . . . . . . 97 


\section{List of Figures}

2.1 Transmission loss in free space at different frequencies with respect to $2 \mathrm{GHz} \ldots \ldots \ldots \ldots \ldots 17$

2.2 Knife-edge diffraction scenarios . . . . . . . . . . . . . 20

2.3 Simplified diffraction geometry . . . . . . . . . . . . . 21

2.4 Knife edge diffraction gain . . . . . . . . . . . . . . . . 24

2.5 Cross-section of $n_{t h}$ Fresnel zone . . . . . . . . . . . . . . . 26

2.6 Fresnel Zones illustration . . . . . . . . . . . . . . . 27

2.7 Fresnel Zones scenarios . . . . . . . . . . . . . . . . . . . . 28

2.8 Geometry for the two-ray model . . . . . . . . . . . . . . . . 30

3.1 Configuration of a bistatic sounder system . . . . . . . . . . . 33

3.2 Radio channel measurement system based on a pulse transmission . . 36

3.3 Radio channel measurement system based on frequency sweeping . . . 36

3.4 Block diagram of the radio channel measurement system . . . . . . . 38

3.5 Back-to-Back VNA-based channel sounder system insertion loss at 2.35 GHz: (a) magnitude, (b) phase . . . . . . . . . . . . . . . 39

3.6 Back-to-Back VNA-based channel sounder system impulse response estimate at centre frequency $2.35 \mathrm{GHz}$. . . . . . . . . . . . 41

3.7 Back-to-Back IREs: (a) $2.35 \mathrm{GHz}$, (b) $18 \mathrm{GHz}$, (c) $28 \mathrm{GHz}$. . . . . . 43 
3.8 The power in IREs estimated from back-to-back measurements as a function of VNA receive subsystem input power in the $2 \mathrm{GHz}$ band . 49

3.9 Block diagram of the $\mathrm{CW}$ transmitter . . . . . . . . . . . . . 53

3.10 Block diagram of the $\mathrm{CW}$ receiver . . . . . . . . . . . . . . 54

4.1 Room layout for empty room measurements . . . . . . . . . . . 57

4.2 Received power as a function of Tx-Rx separation in an empty room. Black: Friis' equation, Red: measurements, Blue: raytracing . . . . . 58

4.3 Static RMS delay spread as a function of Tx-Rx separation of 2-30 GHz frequency range . . . . . . . . . . . . . . . . . 59

4.4 Room layout - single obstruction measurements . . . . . . . . . . . . 60

4.5 $L_{U G A}$ vs Tx-Rx antenna separation at specified centre frequencies for the case of an empty room and when a book shelf cabinet is positioned at $162.5 \mathrm{~cm}$ from an omnidirectional Tx antenna . . . . . . . . . . 61

4.6 Room layout - single obstruction measurements with a reflector against

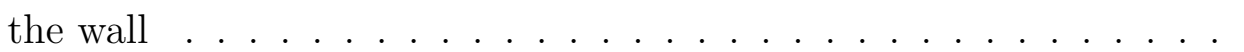

4.7 $L_{U G A}$ as a function of azimuth angle for the indirect radio path around an obstruction at $10 \mathrm{GHz}$ for the case of bare walls and for different targets placed against the wall. . . . . . . . . . . . . .

4.8 (a) Received powers and (b) $L_{U G A}$ values from an azimuth scan in the

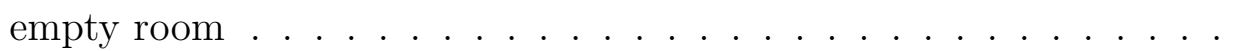

4.9 (a) Received powers and (b) $L_{U G A}$ values from an azimuth scan in the case of one obstruction and bare walls . . . . . . . . . . . . . 
4.10 (a) Received powers and (b) $L_{U G A}$ values from an azimuth scan in the case of one obstruction and a computer monitor against the right side

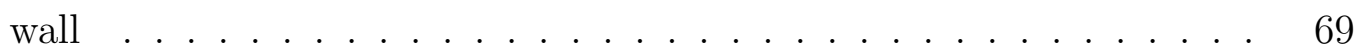

4.11 IREs for the case of one obstruction and bare walls $\ldots \ldots \ldots$. . . 71

4.12 IREs for the case of one obstruction and a reflector placed against the

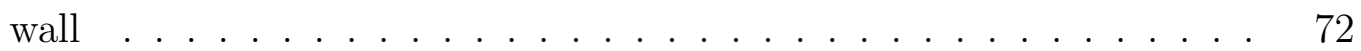

4.13 Room layout - two-obstruction measurements . . . . . . . . 75

4.14 (a) Received powers and (b) $L_{U G A}$ values from an azimuth scan in the case of two-obstruction measurements . . . . . . . . . . . . 77

4.15 IREs for the case of a $15 \mathrm{~cm}$ opening between two book cases placed symmetrically about LoS between the Tx and Rx . . . . . . . 79

4.16 Photograph showing the furniture in the room and the $2-18 \mathrm{GHz}$ horn mounted on the pan-tilt unit . . . . . . . . . . . 81

4.17 Photograph showing the furniture in the room and the biconical antenna 81

4.18 Coverage measurement locations in the cluttered room . . . . . . 82

4.19 Photograph showing the mounted location of the Tx antenna . . . . 83

4.20 Values of $L_{U G A}$ and free space loss in the $2 \mathrm{GHz}$ band as per Scenario 184

4.21 Photograph showing the view from the Tx horn antenna location above the suspended ceiling $\ldots \ldots \ldots \ldots \ldots \ldots$

4.22 Colour-coded values of $L_{U G A}$ as a function of $\mathrm{Rx}$ antenna pointing direction at $18 \mathrm{GHz}$ with illumination by the emulated sector antenna above the ceiling tiles $\ldots \ldots \ldots \ldots \ldots \ldots$

4.23 Transmission loss measurements and modelling results at $2 \mathrm{GHz} \ldots 87$

4.24 Transmission loss measurements and modelling results at $18 \mathrm{GHz} \quad$. 88 
4.25 Transmission loss measurements and modelling results at $28 \mathrm{GHz}$. . 88

4.26 ECDF and KS test results for Gaussian model on $2 \mathrm{GHz}$ channels . . 90

4.27 ECDF and KS test results for Gaussian model on $18 \mathrm{GHz}$ channels . 90

4.28 ECDF and KS test results for Gaussian model on $28 \mathrm{GHz}$ channels . 91

4.29 Static RMS delay spreads for the 3 frequency bands studied as a function of UE location . . . . . . . . . . . . . . . . . . . . . . . . . . 92

4.30 Complementary CDFs of RMS delay spreads computed over all mea-

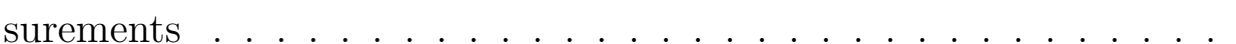

4.31 The number of different directions at UE locations in which a received power within $3 \mathrm{~dB}$ of the power received in the direction of the maximum can be received: (a) $18 \mathrm{GHz}$, (b) $28 \mathrm{GHz}$. . . . . . . . . . . . . 94

4.32 Calibration curve for the CW measurement system . . . . . . . . . 99

4.33 Scatter plots (a) for small sample spacing, (b) for adequate sample

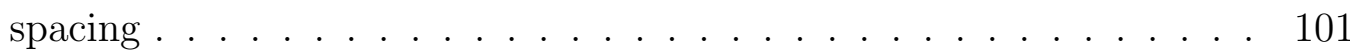

4.34 ECDFs used in two-sample KS test . . . . . . . . . . . . . . . . . 101

4.35 Dynamic ranges for identified fading intervals . . . . . . . . . . . . . 102

4.36 An example result of received signal variations over time with dynamic range of $5 \mathrm{~dB} \ldots \ldots \ldots \ldots$

4.37 An example result of received signal variations over time with dynamic range of $30 \mathrm{~dB} \ldots \ldots \ldots \ldots$

4.38 ECDF and KS test results for Rayleigh model and Ricean model $\mathrm{K}=$

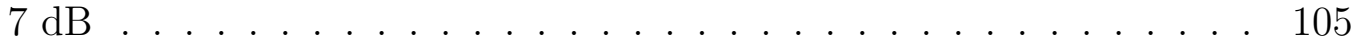

5.1 Distributed antenna system model . . . . . . . . . . . . . . . . 108

5.2 A flow chart illustrating the system capacity simulation steps . . . . . 112 
5.3 ECDF and KS test results for log-normal distribution with 0 mean and $4.4 \mathrm{~dB}$ standard deviation . . . . . . . . . . . . . . . . . . 114

5.4 A scatter plot showing an approximate independence required for the KS-test . . . . . . . . . . . . . . . . 115

5.5 A plot showing ECDFs for simulated data and CDFs models for Rayleigh, Rice $\mathrm{K}=8 \mathrm{~dB}$, Rice $\mathrm{K}=20 \mathrm{~dB} \quad \ldots . \ldots . . \ldots . . \ldots 116$

5.6 A scatter plot showing approximate independence required for the KS-

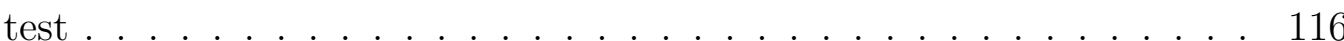

5.7 Scatter plots shows (a) comparison of two uncorrelated Rayleigh envelope sample series, (b) a comparison of two Rayleigh envelope sample series with $50 \%$ correlation . . . . . . . . . . . . . 117

5.8 CDFs for system capacity for IPC in the $18 \mathrm{GHz}$ (Black), and omni 2 GHz (Blue) UC: uncorrelated, TF: temporal fading . . . . . . . . . . 119

5.9 CDFs for system capacity for IPC in the $18 \mathrm{GHz}$ (Black), and omni 2 $\mathrm{GHz}$ (Blue) with 50\% correlation in both shadowing and fading . . . 121

5.10 CDFs for system capacity for IPC in the $28 \mathrm{GHz}$ (Black), and omni 2

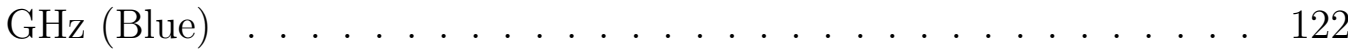

5.11 CDFs for system capacity for IPC in the $28 \mathrm{GHz}$. . . . . . . . . . . 124

5.12 ECDFs for $G_{\operatorname{mux}}$, the ratio of geometric and arithmetic means of the eigenvalues ........................ . . . 125

5.13 Outage capacity as a function of average SNR for IPC in the $18 \mathrm{GHz}$ band for 3 different scenarios . . . . . . . . . . . . . . . 126

B.1 Relationship between correlation coefficients of complex Gaussian $\rho_{g_{i j}}$ and Rayleigh $\rho_{r_{i j}} \ldots \ldots \ldots \ldots$. . . . . . . . . . . . . . 143 
B.2 Simulated bursty Rayleigh envelop fading . . . . . . . . . . . . . . 145 


\section{Acronyms}

4G 4th Generation.

5G 5th Generation.

BS Base Station.

C-MIMO Centralized Multiple-Input-Multiple-Output.

CAS Centralized Antenna System.

CSI Channel State Information.

CW Continuous Wave.

D-MIMO Distributed Multiple-Input-Multiple-Output.

DAQ Data Acquisition Device.

DAS Distributed Antenna System.

DAU Distributed Antenna Unit.

DPC Direct Path Communication.

DUT Device Under Test. 
EDOF Effective Degree of Freedom.

EIRP Effective Isotropic Radiated Power.

FSPL Free Space Path Loss.

GPIB General Purpose Interface Bus.

IFT Inverse Fourier Transform.

IL Insertion Loss.

IPC Indirect Path Communication.

IPLV Indirect Path Loss Variation.

IRE Impulse Response Estimate.

LMS Least Mean Square.

LoS Line of Sight.

MIMO Multiple-Input-Multiple-Output.

MMSE Minimum Mean Square Error.

mmWave Millimetre Wave.

NLoS Non-Line of Sight.

PDP Power Delay Profile.

PLE Path Loss Exponent. 
PTU Pan-Tilt Unit.

RMSE Root Mean Square Error.

$\mathbf{S}_{D}$ RMS delay spread.

SHF Super High Frequency.

SNR Signal to Noise Ratio.

SSSpV Small Scale Spatial Variation.

UE User Equipment.

VCO Voltage-controlled Oscillator.

VNA Vector Network Analyzer.

WiFi Wireless Fidelity. 


\section{Chapter 1}

\section{Introduction}

\subsection{Thesis Background}

Over the past few years, there has been an explosive increase in wireless data traffic as a consequence of a growth in the use of wireless devices such as smartphones and Wireless Fidelity (WiFi) enabled devices. Numerically, according to [1], wireless data traffic is projected to increase by 1000 fold by the year 2020 and probably by 10,000 fold by 2025. Additionally, overall mobile data traffic is expected to grow to 30.6 exabytes per month by 2020, and the smartphones will account for $80 \%$ of the data traffic. Another important factor contributing to the increase in mobile data traffic is the ever increasing number of advanced applications and services that have become an indispensable part of the daily life routine. The advanced applications and services typically require high data rates, and hence, entail a signicant increase in mobile data traffic. This increase has created congestion in the sub-6 GHz frequency bands [2]. Therefore, studying how more wireless channels can be made available, with better

reliability and higher capacity is crucial. Propagation measurements at frequency bands above $6 \mathrm{GHz}$ are first needed to accurately characterize and model the channels 
in order to design capable indoor systems.

Additionally, there is great interest in capacity-enhancing techniques like the use of distributed antenna systems (DASs), which include multiple-input-multiple-output (MIMO) technology. When MIMO is used in spatial multiplexing mode, it takes advantage of the time-varying characteristics of multiple physical links between Tx and Rx antennas to provide multiple independent streams. However, due to the close proximity of antennas in a co-located antenna array, real-world conditions compatible with this assumption are difficult to find, especially when narrow beam antennas are used. As an alternative to co-locating antennas in a MIMO system, distributing antennas over a large area is an appealing solution to the aforementioned problem.

\subsection{Thesis Motivation}

The motivation behind the work that was conducted during this thesis project can be summarized as:

- The primary motivation is to allay the fears that the increased propagation loss resulting from theoretical studies is prohibitive.

- Due to the growth of the considerable number of wireless data subscribers and applications that require high bandwidth capacities [3], the need for larger bandwidth arises. Since bandwidth is limited in the available wireless frequency bands below $6 \mathrm{GHz}$, it is crucial to open up more wireless channels. However, it is anticipated that to provide realistic coverage at frequencies above $6 \mathrm{GHz}$, increased transmission and obstruction loss should be considered. Thus, as a first step, radio channel characteristics of the targeted frequency bands need 
to be studied and compared against the known characteristics of the $2 \mathrm{GHz}$ channels.

- The rapid growth of indoor users in mobile communications systems is expected to continue in the upcoming fifth generation (5G) mobile communication systems. A critical effort in this direction is to gain knowledge at super high frequencies SHF (6 - $30 \mathrm{GHz})$ in different environments such as office buildings.

- The short wavelengths at these high frequencies make the use of many antenna elements in a relatively small form factor possible. The antenna arrays can be used to create highly directional beams that focus transmitted RF energy to overcome the propagation and obstruction loss challenges in both the uplink and downlink.

- Directional beams can also allow the use of reflected signals to maintain a link to a wireless device even when it moves entirely out of the line of sight (LoS) of the transmitter. This can radically expand the role of high-frequency bands for $5 \mathrm{G}$ wireless broadband [4].

- Initial theoretical and experimental investigations suggest that distributed antenna systems can mitigate signal attenuation and dramatically increase achievable rates [5].

\subsection{Thesis Contributions}

The focus of this thesis was on verifying that by taking advantages of specific physical phenomena, indoor communication systems can be designed at high frequency bands, in this case $18 \mathrm{GHz}$ and $28 \mathrm{GHz}$, without future technology innovations to 
yield similar performance, i.e. similar system capacity, as current systems at $2 \mathrm{GHz}$. In this respect, studying and comparing indoor radio channel characteristics at the frequency band of $2 \mathrm{GHz}$ and at the frequency bands of $18 \mathrm{GHz}$ and $28 \mathrm{GHz}$ was a key. By making this comparison, the known $2 \mathrm{GHz}$ band was compared against the aforementioned unknown bands. Also, the conjectured idea of taking advantages of the enabling phenomena to design viable indoor wireless systems at those high frequency bands was verified. It was found that such a comparison study has been addressed in ray tracing studies, but never verified experimentally [6], therefore, the results reported in this thesis are considered to be a worthwhile contribution in the field.

The primary contributions are as follows:

- Indirect path loss models for indoor cluttered environment: Indirect path loss models are useful for modelling systems that may use beam steering at a specific pointing direction to avoid high obstruction losses at SHF (6-30 GHz). In that regard, previous research reports focused on outdoor environment at frequency bands of $28 \mathrm{GHz}, 38 \mathrm{GHz}, 60 \mathrm{GHz}$, and $73 \mathrm{GHz}$ [2], [7], [8]. The only work that provided insight into indirect path loss models in indoor channels at 28 $\mathrm{GHz}$ and $73 \mathrm{GHz}$ only considered the azimuth plane, thus, the $\mathrm{Rx}$ antenna pointing direction was fixed in elevation [9]. Also, this had not been modelled for frequency band of $15 \mathrm{GHz}$ previously which is a band being proposed for some applications ${ }^{1}$. In this work the azimuth and elevation planes were considered in modelling the indirect path loss in indoor channels at both $18 \mathrm{GHz}$ and 28 GHz.

\footnotetext{
$118 \mathrm{GHz}$ band was considered in this thesis instead of $15 \mathrm{GHz}$ since the selected Rx horn operates in the the range of $18-40 \mathrm{GHz}$, $18 \mathrm{GHz}$ band was considered in this thesis instead of $15 \mathrm{GHz}$ since the selected $\mathrm{Rx}$ horn
it is believed that measurement results at this band would still be applicable at $15 \mathrm{GHz}$ band
} 
- Multipath delay dispersion statistics for $18 \mathrm{GHz}$ and $28 \mathrm{GHz}$ when using receiver antenna pointing angles that results in the strongest received power.

- Using frequency averaging technique to mitigate multipath effects: In many research reports of radio propagation measurements and channel modelling, small-scale spatial variations that result from multipath interference are ignored. In other cases, expensive equipment is used to move antennas in local area of radius 1 wavelength for averaging [10]. In this work, it was shown that averaging over frequency is effective at no extra cost.

- Temporal variations characteristics for indoor channels when narrow beam RX antenna is used while pointing toward the angles that result in the strongest received power: This is considered essential in the successful design of future wireless systems in frequency bands above $6 \mathrm{GHz}$ if indirect path communication systems to be designed. However, previous researches had only used omnidirectional antennas when studied indoor radio propagation channel's temporal variations [11], [12].

- Performance evaluation of multi-user Distributed MIMO system simulated using propagation models: Although D-MIMO system capacity has been extensively studied and reported in the literature [13], [14], employing propagationmeasurement-based models in D-MIMO system capacity simulation have not been found in the literature.

\subsection{Literature Review}

\subsubsection{Radio Propagation and Channel Measurements}




\title{
Below $6 \mathrm{GHz}$
}

The $2.4 \mathrm{GHz}$ and $5 \mathrm{GHz} \mathrm{WiFi}$ bands have been widely used for indoor wireless communications in a typical office environment. There has been a massive number of studies of radio propagation and channel models in the sub-6 GHz band over the last 25 years.

Measurements at $910 \mathrm{MHz}$ in an indoor office building has been reported in [15]. The study used continuous wave (CW) tone system to transmit $500 \mathrm{~mW}$ via omnidirectional Tx antenna, and received from a quarter-wave monopole $\mathrm{Rx}$ antenna. Results indicated that in LoS environments, propagation loss over distance closely followed Friis' equation where propagation signals attenuate following the square power law. Rappaport et al. conducted wideband multipath measurements at $1300 \mathrm{MHz}$ in a factory building with 10 ns transmitting pulse. Using Tx and Rx discone antennas, both LoS and NLoS scenarios have been covered and resulted in path loss attenuation of $22 \mathrm{~dB}$ per decade of distance and an RMS delay spread that ranged from $30 \mathrm{~ns}$ to 300 ns [16]. A vector network analyzer (VNA) channel sounder with omni Tx and Rx antennas were used to record the channel transfer function by applying frequency sweeps between 1 and $5 \mathrm{GHz}$ and 5 and $9 \mathrm{GHz}$. Resulted path loss exponent (PLEs) were 1.4 for the LOS scenario, 3.2 for the soft-NLoS, and 4.1 for the hard-NLoS.

\begin{abstract}
Above $6 \mathrm{GHz}$
Dense deployment of indoor hotspots and new multimedia devices have led to increased congestion and traffic over indoor network, one proposal is to open frequency bands in the 6-70 GHz range; however, academic study is needed to characterize and accurately model indoor channels at these new frequency bands. Since the early 1990s,
\end{abstract}


many studies at frequency bands above $6 \mathrm{GHz}$ for indoor environments have been conducted. The $60 \mathrm{GHz}$ band has been used for wireless Gigabit Alliance (WiGig) to support high data rate application. The broadly available bandwidth at $60 \mathrm{GHz}$ band motivated extensive indoor radio propagation measurements to understand channel characteristics necessary for designing capable indoor systems. However, fewer studies have been done at other bands.

Using broadband vector sliding correlator channel sounder, Anderson et al. reported power delay profiles (PDPs) at $2.5 \mathrm{GHz}$ and $60 \mathrm{GHz}$ [17], [18]. For the $60 \mathrm{GHz}$ measurements, vertically polarized pyramidal horn antennas with $25 \mathrm{dBi}$ of gain and an half power beam width (HPBW) of $50^{\circ}$ were used at both the transmitter and receiver. Eight transmitter locations and 22 receiver locations were selected. Tx-Rx separation distances ranged from 3.5 to $27.4 \mathrm{~m}$ on the same floor in a modern office building with a variety of obstructions in the signal path. The location of the Tx and $\mathrm{Rx}$ were chosen to represent a wide range of typical office femto-cellular propagation environments. The height of the transmitter and the receiver antennas were $1.2 \mathrm{~m}$ above the floor. By using minimum mean square error (MMSE) fit, the path loss estimate with respect to a $1 \mathrm{~m}$ free space reference distance at $60 \mathrm{GHz}$ was 2.1 with a standard deviation of $7.9 \mathrm{~dB}$.

Wideband channel measurements at $60 \mathrm{GHz}$ using an omnidirectional Tx and Rx antennas were conducted in several rooms for short-range distances [19]. Propagation at $60 \mathrm{GHz}$ was measured using a channel sounder over $500 \mathrm{MHz}$ bandwidth. Median RMS delay spreads from 3 ns to 9 ns were reported, in addition to calculating a path loss exponent PLE of 1.33 relative to a $1 \mathrm{~m}$ free space reference distance and a shadow loss standard deviation of $5.1 \mathrm{~dB}$ across all measurements. Geng et al. 
performed $60 \mathrm{GHz}$ propagation measurements in different indoor environments [20]. The RMS delay spread distribution followed a log-normal distribution and ranged from 3 ns to 80 ns. Based on the directions of arrival DOA measurements, the propagation mechanisms indicated that the direct wave and the first-order reflected waves from smooth surfaces were sufficient in LoS scenario, while in NLoS cases, diffraction was an essential propagation mechanism, and the transmission loss through walls was very high. These result agree with the results reported in this thesis in which obstruction loss is high at frequency bands above $6 \mathrm{GHz}$ and reflection and diffraction mechanism are necessary for indirect path communication (IPC). PLEs also were reported based on measurements conducted in a corridor, LoS hallway, and NLoS hallway environments, and they are 1.6, 2.2, and 3.0, respectively.

Aside from the majority of indoor propagation research at $60 \mathrm{GHz}$, little is known about other high bands. Lei et al. considered $28 \mathrm{GHz}$ channel propagation measurements in an indoor environment with a VNA and a pair of $26 \mathrm{dBi}$ gain horn antennas for distances up to $30 \mathrm{~m}$. Path loss attenuation slopes as a function of log-distance in different indoor environments were estimated to be 2 in free space, 2.2 in a hallway, 1.2 in a corridor, and 1.8 in an office [21]. The path loss slope results for the office environment contradict with the results reported in this thesis, however, the results from this study cannot be trusted since no averaging was considered to mitigate the multipath interference, therefore, the resulting models can be affected by the multipath propagation. Ultra-wideband propagation measurements were conducted in the $28 \mathrm{GHz}$ and $73 \mathrm{GHz}$ frequency bands in a typical indoor office environment [22]. The measurements conducted using 400 Megachips-per-second broadband sliding correlator channel sounder with rotatable directional horn antennas. After evaluating 
14000 directional power delay profiles measured from unique antenna pointing angles, path loss models, large-scale fading, and multipath time dispersion were reported for co-polarization, cross polarization, and combined-polarization scenarios. Multipath time dispersion statistics showed that the multipath root means square RMS delay spread can be reduced when using transmitter and receiver antenna pointing angles that result in the strongest received power. The path loss models showed that for co-polarized antennas, constructive interference due to waveguiding and reflections resulted in nearly identical LoS PLEs of 1.7 and 1.6 for $28 \mathrm{GHz}$ and $73 \mathrm{GHz}$, respectively; these results are smaller than the theoretical FSPL, suggesting that directional LoS PLEs are independent of frequency. Path loss observed in NLoS environments had much greater attenuation when compared with LoS environments, $44 \mathrm{~dB}$ (PLE $=4.4)$ and $53 \mathrm{~dB}(\mathrm{PLE}=5.3)$ per decade of distance for $28 \mathrm{GHz}$ and $73 \mathrm{GHz}$, respectively. However, the high attenuation was significantly reduced (resulting in $n=3.0$ at $28 \mathrm{GHz}$ and $\mathrm{n}=3.4$ at $73 \mathrm{GHz}$ ), when best Tx and Rx antenna pointing angles that resulted in the maximum received power at each measured location was considered. In this study, only azimuth plane was considered when scanned for the maximum receive power pointing directions, however, it is believed that in a cluttered indoor environment, strong signal reflection can be received from any pointing directions including those coming from elevation angles other than $0^{\circ}$, therefore, results reported in this thesis are considered to be a significant improvement over previous results.

A fundamental threat to the reliability of the aforementioned studies is that none has considered mitigating the effect of multipath interference. In other words, the derived propagation models are high-likely biased by the multipath effects. It is believed that the wide ignorance of mitigating the multipath interference is due to the 
high-cost associated with the high-accuracy mechanical equipment needed to perform this averaging over small local area at high frequency bands. Therefore, one of the significant contribution to the body of knowledge is introducing a new averaging technique that yield similar results as those resulting from applying the well-known averaging-over-space technique.

\subsubsection{Small Scale Fading}

While large-scale path loss model parameters have been extensively studied, the statistics of small-scale fading, especially, at SHF bands have received less attention, however, such statistics information are vital when estimating path amplitude gains in MIMO simulations. Small-scale fading measurements in the UHF and Microwave bands in both indoor and outdoor environments have previously provided vital insight into temporal fading of multipath amplitudes. Using a continuous wave (CW) propagation measurement system, Bultitude conducted measurements at $910 \mathrm{MHz}$ in order to determine the characteristics of indoor radio channels between fixed terminals [15]. His results demonstrate that variations in the received signal envelope are caused by the movement of personnel and machinery. These variations are slow, having a bandwidth of only about $4 \mathrm{~Hz}$, and have characteristics that depend on the interior floor plan of the building. Particularly, fading occurs in bursts when the floor plan is divided into separate rooms, and in that case the dynamic range of fading is $30 \mathrm{~dB}$. However, when people were moving in an open office environment, fading was found to be continuous and has $17 \mathrm{~dB}$ dynamic range. The temporal fading distribution were found to be Ricean-like with $\mathrm{K}$ ratios ranging between $-12 \mathrm{~dB}$ and $-6 \mathrm{~dB}$. 
Ultra-wideband outdoor small scale fading measurements at $28 \mathrm{GHz}$ in LoS and NLoS environments have been conducted and results are reported in [23]. The envelop, under narrow $\mathrm{Tx}$ and $\mathrm{Rx}$ beam pointing direction, was shown to be best characterized by a Ricean distribution, with $\mathrm{K}$ ratios ranging from 9 - $15 \mathrm{~dB}$ and 5 $8 \mathrm{~dB}$ in LoS and NLoS V-V scenarios, respectively, and $3-7 \mathrm{~dB}$ in both LoS and NLoS V-H scenarios. As shown in this study, for wideband mmWave channels, data indicates that the amplitudes are no longer Rayleigh-distributed.

\subsubsection{Distributed-MIMO Systems}

Distributing antennas is an appealing approach to provide reasonably uniform coverage and enable macro-diversity. A comparison between conventional and distributed MIMO systems from a measurement campaign in an indoor environment has been reported in [24]. Different configurations of the transmitter were studied for both systems. The channel sounder used was based on a Multiport Network Analyzer (MNA) and a fast switch. Results showed that capacity obtained from the distributed MIMO system is around the capacity of an independent and identically distributed (i.i.d.) fading channel. Capacity for a fixed SNR of $15 \mathrm{~dB}$ has been calculated. Results showed that distributed systems could achieve a capacity $3 \mathrm{bits} / \mathrm{s} / \mathrm{Hz}$ greater than conventional systems. The capacity behaviour of a D-MIMO system for a composite Rayleigh shadowing and Rician shadowing channel has been studied and reported in [25]. Experimentation for different shadowing intensity levels is provided, showing a wide variety of results of these systems, depending on the number of elements and the SNR. It was identified that the D-MIMO systems could benefit from shadowing in Rician channels. The D-MIMO system with Rician fading and distributed antennas 
that experience log-normal shadowing can exceed the capacity of traditional MIMO systems with Rician only channel. Thus, considering multipath propagation turned out to be the solution to reach higher capacities, the cooperative networks can take advantage of shadowing, which has been typically considered as a restriction to reach higher capacities. A study of the performance of an indoor distributed antenna system (DAS) based on a measured wideband multiple input multiple output (MIMO) channel model constructed with measured data at $5.25 \mathrm{GHz}$ was reported in [26]. In this study, different transmission schemes and antenna congurations were compared. The results showed that the indoor DAS with selection transmission scheme could significantly enhance both the ergodic and outage capacity compared with the conventional antenna system (CAS). The impact of polarization and antenna spacing on MIMO DAS were also investigated. The study also found that the MIMO technique can improve the system performance signicantly even under LoS propagation conditions.

In a DAS, each distributed access unit DAU has different channel gains since uplink/downlink signals between the user terminals and the different antenna ports experience different multipath fading, shadowing and transmission loss characteristics. However, all the other studies that have been reviewed, always, ignored the transmission loss characteristics and consider constant average SNR over all antenna ports. In this work, path loss characteristics have been considered in computing the system capacity. 


\subsection{Thesis Organization}

In Chapter 2, the radio wave transmission impairments focusing on frequency bands above $6 \mathrm{GHz}$ will be discussed. In Chapter 3, the propagation measurement system will be introduced. This chapter is organized into two parts. Part 1 deals with measurement equipment and techniques employed in phase 1 of the project when wideband sounding measurement system was used, and part 2 describes the measurement equipment and techniques of phase 2 of the project when a single tone measurement system was used. In Chapter 4, phenomenological measurements and modelling in an empty room and the characterization of $2 \mathrm{GHz}, 18 \mathrm{GHz}$, and $28 \mathrm{GHz}$ radio channels in a cluttered environment are presented. Multi-user D-MIMO system capacity evaluations for $4 \times 4$ D-MIMO is reported in Chapter 5. Finally, in Chapter 6 , a summary and a conclusion of the results and recommendations for further researches are given. 


\section{Chapter 2}

\section{Physical Phenomena of Particular Interest in Considering Indoor Wireless Communications at Frequencies Between $6 \mathrm{GHz}$ and $30 \mathrm{GHz}$}

Understanding the propagation behaviour of radio waves in various media and the available power to a receiver is key in the successful implementation of any radio system. Transmission loss, also known as path loss or attenuation, refers to the gradual loss of power density of a signal in a medium. When the transmission loss increases, the SNR at a receiver decreases, which in turn limits achievable rates for reliable digital data communications and hence has to be taken into account when designing communication systems. Estimation of transmission loss is important in ensuring an acceptable quality of service by locating transmitters at appropriate locations and with appropriate power levels to provide coverage over the desired area.

This chapter discusses transmission loss in the frequency range of 2-30 GHz starting with the simple case of propagation in free space, followed by the review of various propagation models, which include a diffraction loss model, and the effect of reflections in the presence of a direct path between the transmitter and receiver. Multipath 
propagation is then discussed for indoor area.

\subsection{Large-Scale Transmission Loss}

Propagation models that predict the mean received power for arbitrary transmitterreceiver $(\mathrm{Tx}-\mathrm{Rx})$ separations distance are useful in estimating the radio coverage area of a transmitter and called large-scale propagation models.

\subsubsection{Free Space Transmission}

The free space transmission loss equation is derived under the far field assumption where the propagation distance $d$ that separates the transmitter and receiver is much larger than $D$ which is the largest physical linear dimension of the antenna $2 D^{2} / \lambda[27]$.

1. $d>d_{f}$, where $d_{f}$ is the Friis distance, also known as the Fraunhofer distance or the Rayleigh distance.

2. $d_{f}>\frac{2 D^{2}}{\lambda}$ and $d_{f} \gg \lambda$.

3. The transmit antenna is an ideal isotropic antenna (unity gain).

Under these assumptions the radiated energy is spread over the surface of a sphere, where the power density $S$ in units of watts per $m^{2}\left(\mathrm{~W} / \mathrm{m}^{2}\right)$ at distance $d$ is equal to $S=P_{T} / 4 \pi d^{2}$ where $4 \pi d^{2}$ is the surface area of the sphere of radius $d$ and $P_{T}$ is the transmitted power in (watts). The power density decreases as the distance increases away from the source. The available power $P_{R}$ in watts $(\mathrm{W})$ at the receive antenna with effective area $A_{e}$, is given by

$$
P_{R}=\frac{P_{T}}{4 \pi d^{2}} A_{e} .
$$


The effective area of the antenna $A_{e}$ is the area of a circle constructed broadside to the incoming radiation where all radiation passing through the circle is delivered to a matched load, and it is given by

$$
A_{e}=\frac{\lambda^{2}}{(4 \pi d)^{2}} P_{T} G_{R},
$$

where $\lambda$ is the wave length, and $G_{R}$ is the receive antenna gain. The size of a resonant antenna is inversely proportional to the frequency, and same applies for the effective area of the antenna. Therefore, radiation at frequencies above $6 \mathrm{GHz}$ suffers from extra transmission loss caused by smaller effective area $A_{e}$. For unity gain antennas, at different frequencies $f_{i}$ and $f_{j}$, the difference in transmission loss at two frequencies is

$$
\Delta L_{i j}=\frac{\lambda_{i}^{2}}{\lambda_{j}^{2}}
$$

Figure 2.1 shows the difference of transmission loss between equal gain antennas at the specified frequencies and at $2 \mathrm{GHz}$. It can be seen that the transmission loss increases quadratically as frequency increases. 


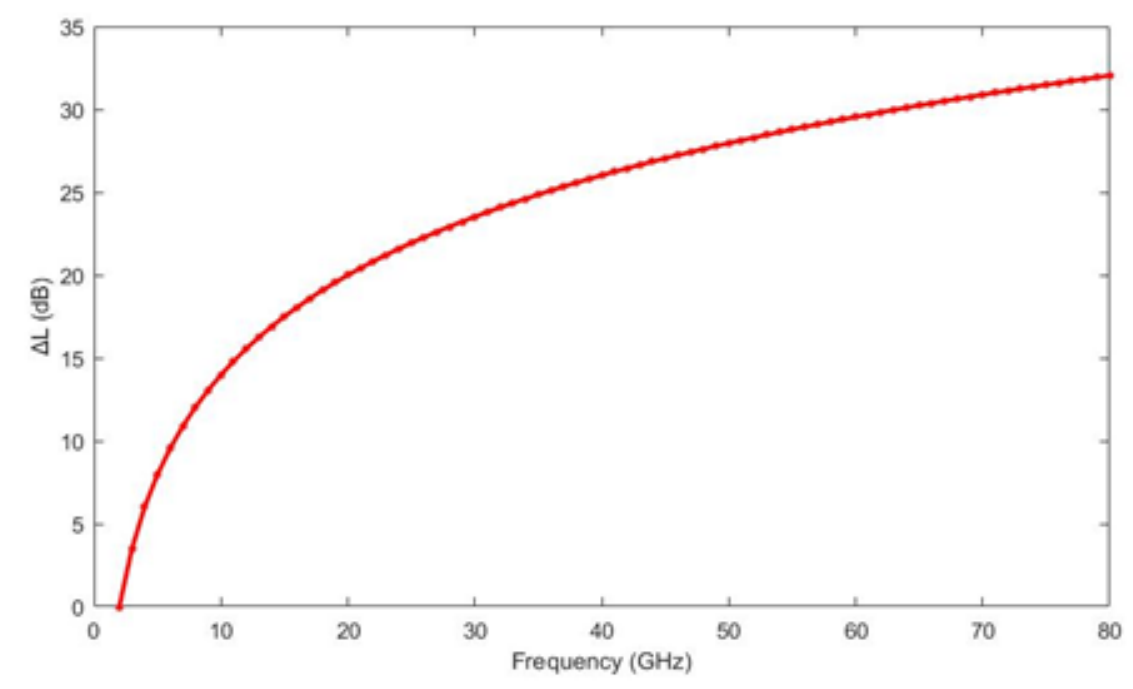

Figure 2.1: Transmission loss in free space at different frequencies with respect to 2 $\mathrm{GHz}$

For unity gain antenna, transmission loss can be expressed as the ratio of the transmitted power to the received power as follows

$$
\frac{P_{T}}{P_{R}}=\left[\frac{4 \pi d f}{c}\right]^{2}
$$

where $f$ is the frequency in $(\mathrm{Hz})$ and $c$ is the speed of light $(\mathrm{m} / \mathrm{s})$. This equation can be expressed in decibels $(\mathrm{dB})$ as follows

$$
\begin{gathered}
L_{f}=10 \log _{10}\left(\frac{P_{T}}{P_{R}}\right)=20 \log _{10} f+20 \log _{10} d+k \\
k=-20 \log _{10}\left(\frac{c}{4 \pi}\right)=-147.6
\end{gathered}
$$

All the equations above are for unity gain antennas, however, when the transmit and receive antennas have gain higher than unity, i.e., $G_{T}$ and $G_{R}$, Eq (2.6) becomes: 


$$
\frac{P_{T}}{P_{R}}=\frac{1}{G_{T} G_{R}}\left[\frac{4 \pi d}{\lambda}\right]^{2}
$$

Then the received signal power in decibels becomes:

$$
P_{R}=P_{T}+G_{T}+G_{R}-L_{f}=E I R P+G_{R}-L_{f}
$$

where EIRP is the effective isotropic radiated power and defined as

$$
\mathrm{EIRP}=P_{T}+G_{T}
$$

It can be seen from the previous equations that the transmission loss increases with the square of the transmission frequency. An increase in antenna gain can be used to compensate for the extra transmission loss due to the decrease of collected power caused by smaller antenna aperture. Since the antenna does not create energy, an increase in antenna gain means that the power is focused in specific direction. This section can be concluded as follows

In free space:

1. Transmission loss is not affected by any property of the transmission medium.

2. Transmission loss can only be changed by changing $G_{T}, G_{R}, \lambda$ or $d$.

3. For frequency bands above $6 \mathrm{GHz}$, high-gain is needed and this can normally only be accomplished by using narrow beamwidths (radiation pattern is like a balloon when pushed in one side gives narrow beam elsewhere). 


\subsection{Diffraction Loss}

Diffraction is the bending of wave-fronts around obstacles such as hills, rooftops, or furniture when they fall in between the Tx and Rx antennas [27]. Diffraction always occurs, however, its effects are generally noticeable only for waves where the wavelength similar to, or larger than the dimensions of the diffracting objects. It can be explained by using Huygens principle, which says that all points on a wavefront can be considered as points for the production of secondary wavelets, which subsequently combine to produce new waves in new directions. Hence, even if a region is shadowed by an obstruction, diffraction around an obstruction produces waves that propagate into the shadowed region.

\subsubsection{Knife-Edge Diffraction}

A simplified scenario that is used in chapter 4 to illustrate diffraction effects at different frequencies called knife-edge diffraction is considered. In this scenario the obstruction is considered to be a knife-like edge protruding into the path between the Tx and Rx antennas. The geometry is shown in Figure 2.2(a) [27]. In this figure the $T$ and $R$ represent Tx and Rx antennas, respectively. The obstruction is assumed to have infinite width (i.e. it extends infinitely into and out of the page). No signals can penetrate the obstruction; therefore, some of the rays emanating from the Tx will not reach the Rx. However, in an imaginary plane located in line with the obstruction, points above the obstruction can be considered secondary sources of wavelets, which combine to form waves propagating toward the $\mathrm{Rx}$ to the right of the obstruction. By superposition, rays produced by all the sources above the obstruction are all added and the total field produced at the $\mathrm{Rx}$ is found. 
(a)

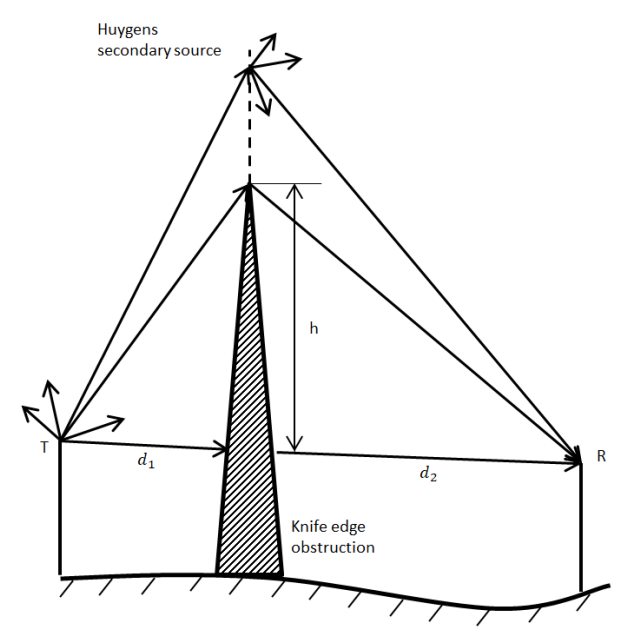

(b)

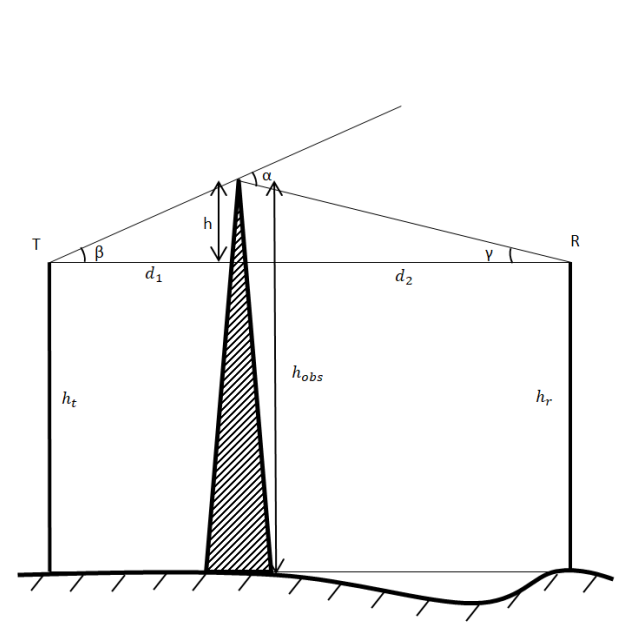

Figure 2.2: Knife-edge diffraction scenarios

By looking into the geometric situation shown in Figure 2.2 (b), the obstruction has a height $h_{o b s}$, and the Tx and Rx antennas are at heights $h_{t}$ and $h_{r}$, respectively. Two propagation paths from the Tx antenna to the $\mathrm{Rx}$ antenna are considered: a direct path through the obstruction and a diffracted path. By drawing the direct path from the Tx antenna to the Rx antenna, the Tx antenna is at a distance $d_{1}$ from the obstruction and the $\mathrm{Rx}$ antenna is at a distance $d_{2}$ from the obstruction, along this line. The diffracted path makes an angle $\beta$ with the horizontal on the Tx antenna side and an angle $\gamma$ with the horizontal on the Rx antenna side. As the Tx and Rx antennas are at different distances away from the obstruction, these angles are not necessarily equal. For large $d_{1}$ and $d_{2}$, this geometry can be used, which assumed $h_{t}=h_{r}$, to simplify the analysis and it will remain approximately true for $h_{t} \neq h_{r}$, provided the separation distance is large compared to the heights. A simplified illustration is shown in Figure 2.3. 


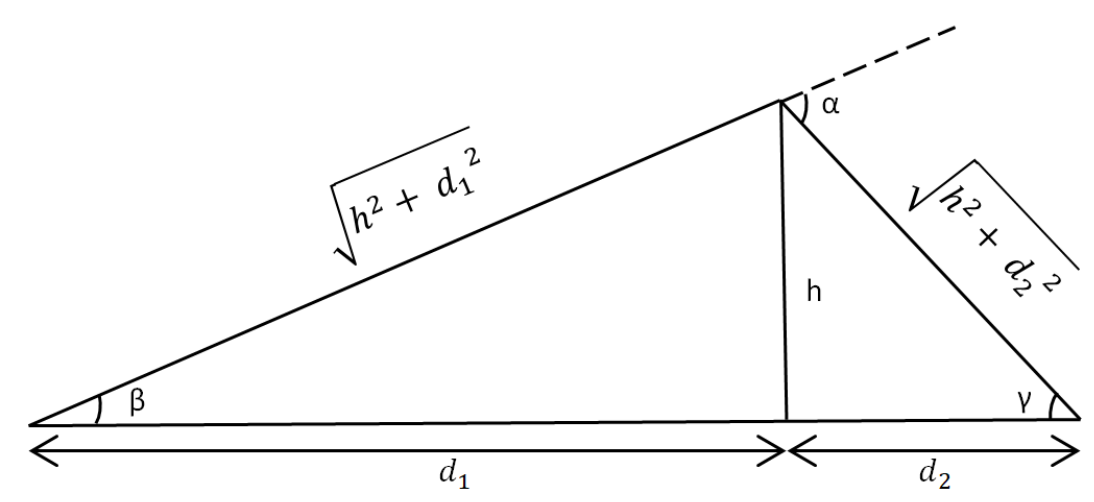

Figure 2.3: Simplified diffraction geometry

The goal is to find the received electric field strength from the diffracted path shown, relative to the electric field strength of the direct path. Its characteristics depend strongly on the path difference $\Delta$ between the length of the diffracted path and the length of the direct path. Using the geometry shown $\Delta$ is easily found as follows

$$
\begin{aligned}
\Delta & =\sqrt{d_{1}^{2}+h^{2}}+\sqrt{d_{2}^{2}+h^{2}}-\left(d_{1}+d_{2}\right) \\
& =d_{1} \sqrt{1+\frac{h^{2}}{d_{1}^{2}}}+d_{2} \sqrt{1+\frac{h^{2}}{d_{2}^{2}}}-d_{1}-d_{2} \\
& \approx d_{1}\left(1+\frac{h^{2}}{d_{1}^{2}}\right)+d_{2}\left(1+\frac{h^{2}}{d_{2}^{2}}\right)-d_{1}-d_{2} \\
& =\frac{h^{2}}{2}\left(\frac{1}{d_{1}}+\frac{1}{d_{2}}\right) \\
& =\frac{h^{2}}{2}\left(\frac{d_{1}+d_{2}}{d_{1} d_{2}}\right)
\end{aligned}
$$

where use is made of the approximation 


$$
\sqrt{1+\epsilon} \cong 1+\frac{\epsilon}{2}
$$

The angle $\alpha=\beta+\gamma$, and if $d_{1}, d_{2} \gg h$,

$$
\begin{aligned}
& \beta=\tan ^{-1} \frac{h}{d_{1}} \approx \frac{h}{d_{1}} \\
& \gamma=\tan ^{-1} \frac{h}{d_{2}} \approx \frac{h}{d_{2}} \\
& \alpha=\beta+\gamma=\frac{h\left(d_{1}+d_{1}\right)}{d_{1} d_{2}} .
\end{aligned}
$$

The electrical length of the path difference is equal to

$$
\Delta \phi=k \Delta=\frac{2 \pi}{\lambda} \frac{h^{2}}{2}\left(\frac{d_{1}+d_{2}}{d_{1} d_{2}}\right)
$$

where $k$ is the angular wave number

$$
v=h \sqrt{\frac{2\left(d_{1}+d_{2}\right)}{\lambda d_{1} d_{2}}}
$$

where $v$ is the Fresnel-Kirchoff parameter and it is the height of the screen above the direct path multiplied by a frequency-dependent scalar. When the edge of the screen is below the direct path, $h$ is negative and $v$ becomes a negative value as a result, one can express $\phi$ as

$$
\Delta \phi=\frac{\pi}{2} v^{2}
$$

When the difference in received electrical field magnitude is assumed to be zero, the path difference for one diffracted ray relative to the direct path becomes 


$$
\frac{E_{d}}{E_{0}}=\exp \left(-j \frac{\pi}{2} v^{2}\right)
$$

where $E_{d}$ is the diffracted field strength and $E_{0}$ is the free space field strength. After adding all Huygens sources above the screen, integration from $v$ to infinity is applied

$$
\frac{E_{d}}{E_{0}}=F(v)=\frac{1+j}{2} \int_{v}^{\infty} \exp \left(-j \frac{\pi}{2} t^{2}\right) d t
$$

To evaluate this equation, the complex exponential is expanded into its trigonometric equivalent where each term can be expressed as

$$
\int_{v}^{\infty} \cos \left(\frac{\pi}{2} t^{2}\right)=\frac{1}{2}-\int_{0}^{v} \cos \left(\frac{\pi}{2} t^{2}\right) d t
$$

and

$$
\int_{v}^{\infty} \sin \left(\frac{\pi}{2} t^{2}\right)=\frac{1}{2}-\int_{0}^{v} \sin \left(\frac{\pi}{2} t^{2}\right) d t
$$

Eq (2.16) can then be rewritten in terms of the real part $C(v)$ and the imaginary part $S(v)$ of the Fresnel integral

$$
C(v)=\int_{0}^{v} \cos \left(\frac{\pi}{2} t^{2}\right) d t
$$

and

$$
S(v)=\int_{0}^{v} \sin \left(\frac{\pi}{2} t^{2}\right) d t
$$

Substituting in Eq (2.16), one can write the normalized electric field as 


$$
\frac{E_{d}}{E_{0}}=F(v)=\frac{1+j}{2}\left\{\left[\frac{1}{2}-C(v)\right]-j\left[\frac{1}{2}-S(v)\right]\right\}
$$

By defining $g_{d i f f}$ as inverse diffraction loss (or gain) as

$$
g_{d i f f}=\frac{1+j}{2}\left|\left[\frac{1}{2}-C(v)\right]-j\left[\frac{1}{2}-S(v)\right]\right|^{2}=\frac{1}{l_{d i f f}}
$$

A plot of $g_{d i f f}$ is illustrated in Figure 2.4.

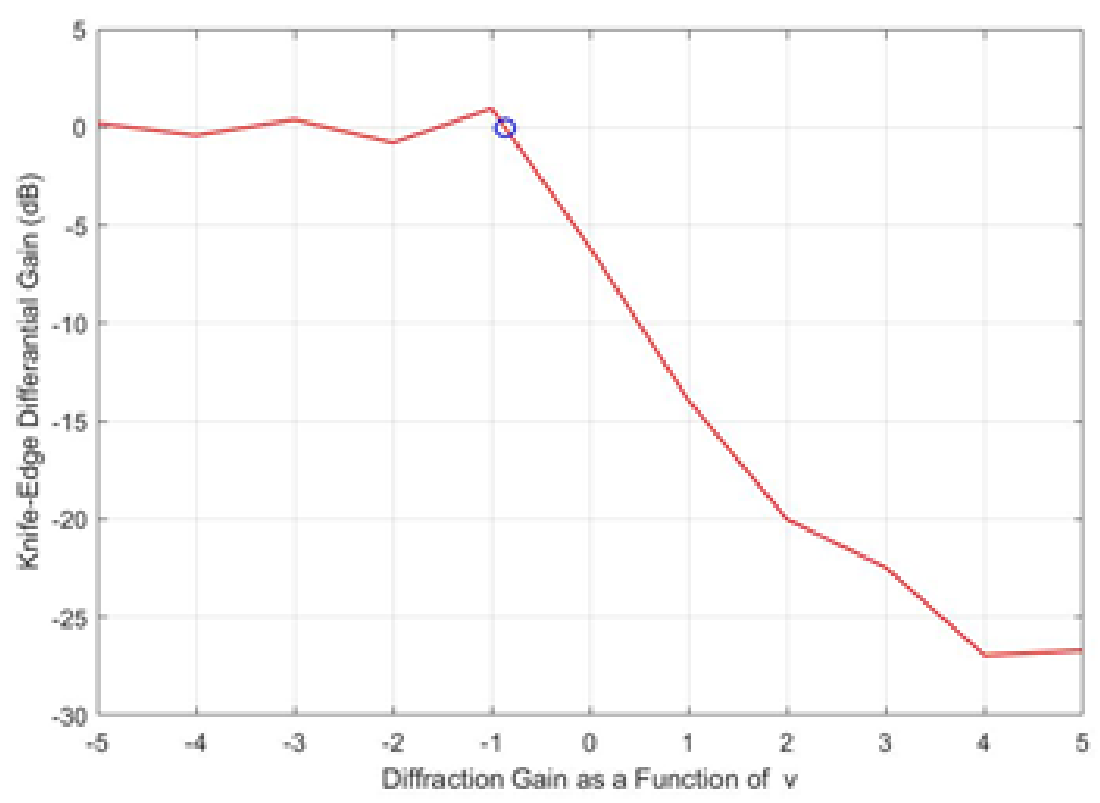

Figure 2.4: Knife edge diffraction gain

A steep drop in $g_{d i f f}$ can be observed as commencing at $v=-1$, which corresponds to $\phi=\frac{\pi}{2}$ or a quarter wavelength of path length difference $h$. Figure 2.4 indicates that there is a need to keep some clearance distance to the first obstacle along the path between the transmitter and the receiver. The amount of this clearance is defined in terms of Fresnel zones, which are regions bounded by ellipsoids of 
revolution whose foci are at the transmitter and at the receiver.

\subsubsection{Fresnel Zones}

Consider the contribution from a single Huygens source in the diffraction problem. It is elevated a distance $h$ from the LoS ray. One can observe that if the height $h$ is chosen such that the path length differential between the diffracted path and LoS path $\Delta$ is one half-wavelength, the diffracted wave has incurred a $180^{\circ}$ phase shift relative to the LoS wave. This is true over a locus of points forming a ring in the plane of the obstruction. Such a ring or circle is called a Fresnel zone [28]. If $h$ is increased further, such that $\Delta=\lambda$, one can get a ring of sources that produce fields in phase with the LoS path at the receiver. This process repeats, giving Fresnel zones that alternately provide constructive and destructive interference to the total received signal every $\frac{\lambda}{2}$ increase in the length of the path taken by the diffracted wave. The set of points at which propagation produces an excess path length of precisely $n \frac{\lambda}{2}$ is called the $n_{t h}$ Fresnel zone. Consider the triangle in Figure 2.5 which shows a cross-section of the $n_{t h}$ Fresnel zone. Path $A B$ is the direct path and path $A C B$ is the indirect path. 


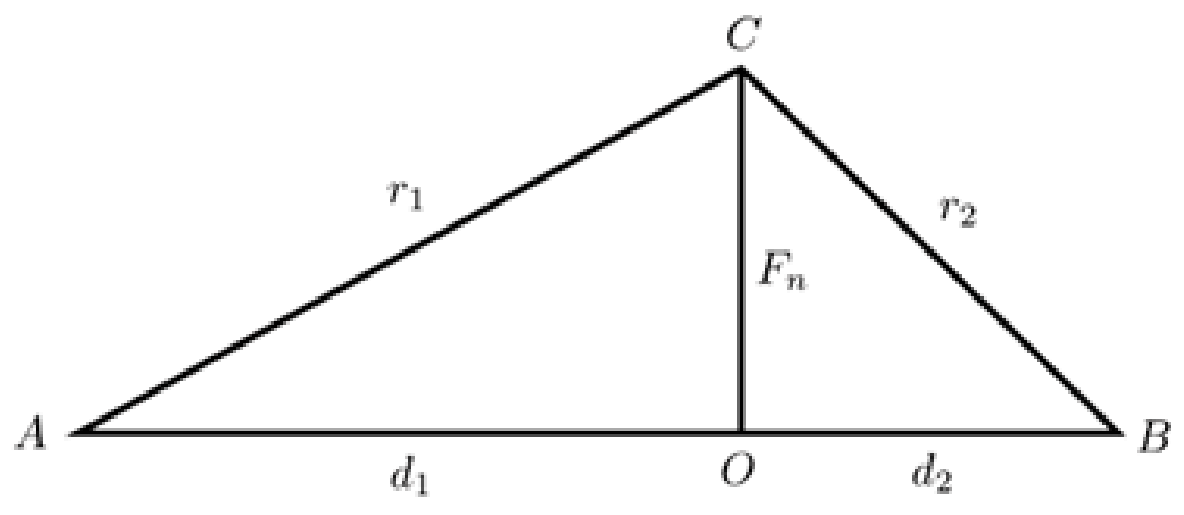

Figure 2.5: Cross-section of $n_{t h}$ Fresnel zone

The condition that will locate point $C$ on the $n_{t h}$ Fresnel zone is

$$
r_{1}+r_{2}=d_{1}+d_{2}+n \frac{\lambda}{2}
$$

hence

$$
\sqrt{d_{1}^{2}+F_{n}^{2}}+\sqrt{d_{2}^{2}+F_{n}^{2}}=d_{1}+d_{2}+n \frac{\lambda}{2}
$$

and since $F_{n} \ll d_{1}, d_{2}$ one can approximate this as

$$
\left(d_{1}^{2}+\frac{F_{n}^{2}}{2 d_{1}}\right)+\left(d_{2}^{2}+\frac{F_{n}^{2}}{2 d_{2}}\right)=d_{1}+d_{2}+n \frac{\lambda}{2}
$$

yielding

$$
F_{n}=\sqrt{\frac{n \lambda d_{1} d_{2}}{d_{1}+d_{2}}}
$$

Each circle of radius $F_{n}$ has an excess path length of $\frac{\lambda}{2}, \lambda, \frac{3 \lambda}{2}$, etc. for $n=1,2,3, \ldots$. Note that $F_{n}$ depends on the distances $d_{1}$ and $d_{2}$ to the obstruction and that $F_{n}$ is 
maximum when $d_{1}=d_{2}$. The circles shrink as the obstruction is moved closer to the transmitter or receiver. If all the points between the transmitter and receiver for which $\Delta$ is an integer multiple of $\frac{\lambda}{2}$ are joined, one can obtain ellipsoids, as shown in Figure 2.6 [28].

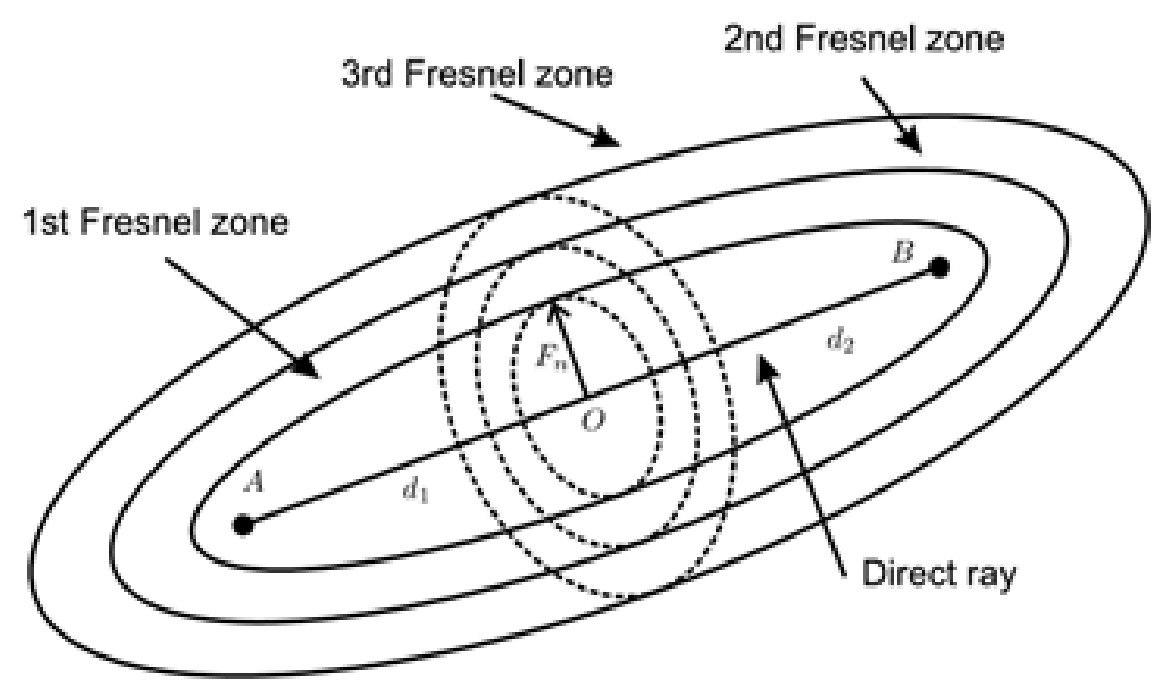

Figure 2.6: Fresnel Zones illustration

The relevance of Fresnel zones to diffraction considerations can be seen in Figure 2.7 [28]. Shadowing is sensitive to frequency, location of obstruction and relative height of transmitter and receiver. The Fresnel-Kirchhoff diffraction parameter can be expressed using $F_{n}$ as

$$
v=\frac{h}{F_{n}} \sqrt{2 n}
$$




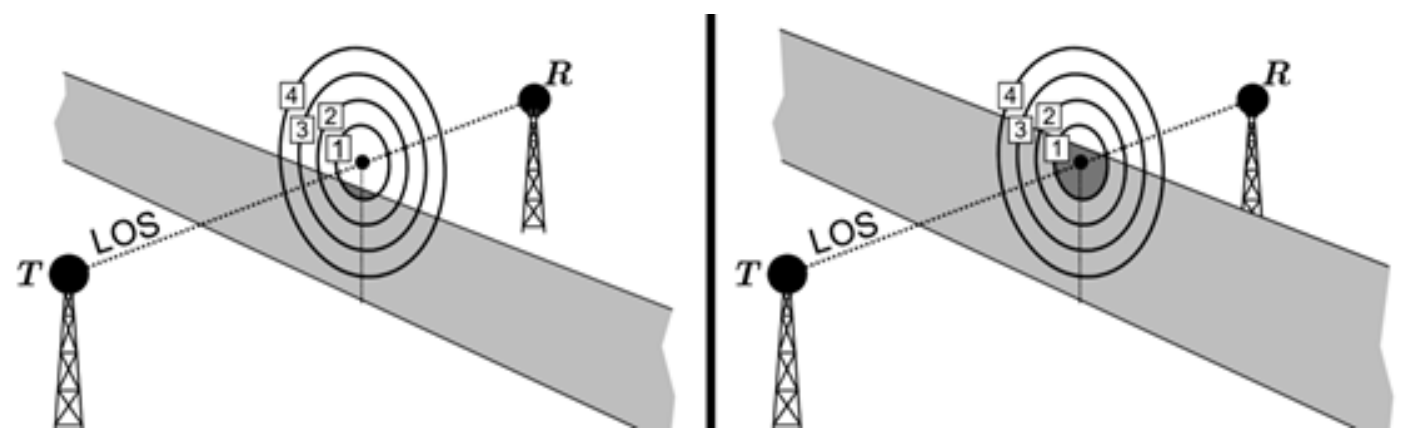

Figure 2.7: Fresnel Zones scenarios

If most of the $1_{s t}$ Fresnel zone is kept clear then the obstacle will cause negligible diffraction losses. However, if the obstacle blocks a significant part of the $1_{s t}$ Fresnel zone, diffraction losses need to be considered. From Figure 2.4, one can observe that if the Fresnel-Kirchhoff diffraction parameter is smaller than -0.8, the obstruction has no significant effect on the electric field strength at the transmitter. This value of corresponds to roughly $60 \%$ of the first Fresnel zone [27]. If this path is kept clear, then the total path attenuation will be practically the same as in the unobstructed case. Thus, it can be observed that when the wavelength of a signal decreases, more objects in the surrounding will form obstacles of that signal. Small objects like a computer monitor or human body will form a significant obstruction for signals at $28 \mathrm{GHz}$ for instance. However, it is also speculated that there should be many more good reflectors in a cluttered indoor environment that can provide more indirect paths around obstructions. This can be explained by considering the inverse relationship between frequency and $1_{s t}$ Fresnel zone radii. For an obstruction to be a good specular reflector, the fraction of its area that intersects with an impinging wave must be large enough (at least larger than $60 \%$ of the $1_{s t}$ Fresnel zone) at the point and angle of intersection. Since $1_{s t}$ Fresnel zone becomes smaller as frequency increases, therefore, 
more objects in the environment become good reflectors.

This section can be concluded as follows:

1. In order to achieve the equivalent of free space transmission loss between two antennas, the $1_{s t}$ Fresnel zone surrounding the two antennas must be free from obstructions over at least $60 \%$ of its radius.

2. As frequency increases, i.e. frequency bands above $6 \mathrm{GHz}$, the $1_{s t}$ Fresnel zone becomes smaller, therefore the traveling wave passes through gaps and opening that are large enough (larger than $1_{s t}$ Fresnel zone) without almost any diffraction loss.

3. A given object becomes a better reflector as frequency increases.

\subsection{Multipath Propagation}

Electromagnetic (EM) waves propagating in one medium and impinging upon another medium with different electrical properties are partly reflected and partly transmitted. An important result of reflection is the reception of multiple waves or signals that are delayed with respect to each other in time and suffer different attenuations at the receiver. Generally, in indoor environment there is large number of reflectors especially for frequency bands above $6 \mathrm{GHz}$. To understand the phenomenon of reflection and its effect on the received signal, a Two-Ray model can be considered.

\subsubsection{Two-Ray Model}

Assume a simple propagation environment where there are two paths overwhich radio waves can travel from Tx antenna to Rx antenna as shown in Figure 2.7 [27]. 


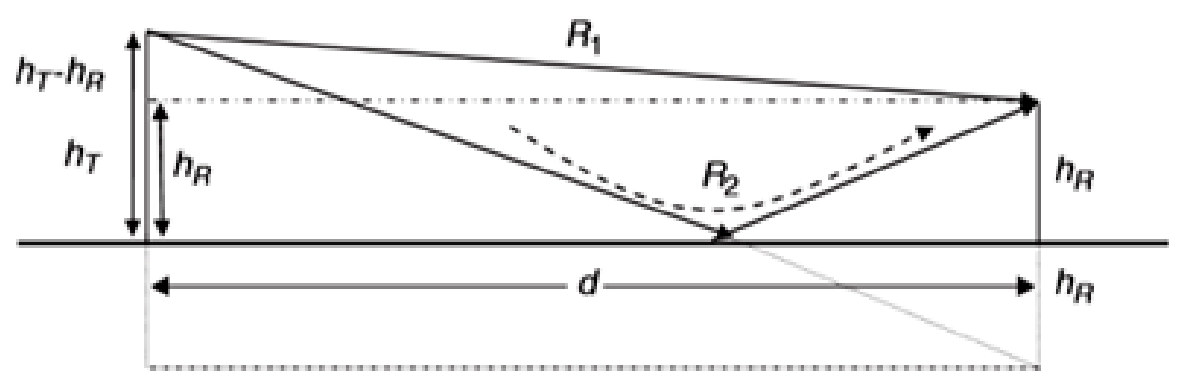

Figure 2.8: Geometry for the two-ray model

This model is used to estimate path loss in environments where there are few obstructions between the transmitter and the receiver. The received signal in this case consists of a direct free space wave with electrical field $E_{0}$ and a reflected wave with electrical field $E_{1}$, and $\rho$ is the reflection coefficient and $\Delta$ is the phase difference between the direct and the ground reflected wave which is proportional to $R_{2}-R_{1}$ in Figure 2.8. Assuming that the distances between the transmitter and receiver $d \ll h_{t}, h_{r}$ and using the approximation $\sqrt{1+\epsilon} \cong 1+\frac{\epsilon}{2}$, expressions for $R_{1}$ and $R_{2}$ can be found to be

$$
\begin{aligned}
& R_{1}=\left[d^{2}+\left(h_{T}-h_{R}\right)^{2}\right]^{\frac{1}{2}}=d\left[1+\frac{\left(h_{T}-h_{R}\right)^{2}}{d^{2}}\right]^{\frac{1}{2}} \cong d\left[1+\frac{1}{2}\left(\frac{h_{T}-h_{R}}{d}\right)^{2}\right] \\
& R_{2}=\left[d^{2}+\left(h_{T}+h_{R}\right)^{2}\right]^{\frac{1}{2}}=d\left[1+\frac{\left(h_{T}+h_{R}\right)^{2}}{d^{2}}\right]^{\frac{1}{2}} \cong d\left[1+\frac{1}{2}\left(\frac{h_{T}+h_{R}}{d}\right)^{2}\right]
\end{aligned}
$$

Using $\mathrm{Eq}(2.27)$ and $\mathrm{Eq}(2.28)$, the difference $\Delta R=R_{2}-R_{1}$ is found to be 


$$
\Delta R \approx \frac{2 h_{T} h_{R}}{d}
$$

The phase difference can then be expressed as

$$
\Delta \approx \frac{4 \pi}{\lambda} \frac{h_{T} h_{R}}{d}
$$

\subsection{Small Scale Fading and Multipath}

The term small scale fading, or simply fading, is used to describe the rapid fluctuation of the amplitude of a radio signal over a short period of time or travel distance. Fading is caused by constructive and destructive interference between two or more versions of the transmitted signal being slightly out of phase due to the different propagation delay. Since the phases are random, the sum of the contributions varies widely; for example, obeys a Rayleigh fading distribution. In small-scale fading, the received signal power may vary by as much as three or four orders of magnitude (30 or $40 \mathrm{~dB}$ ) when the receiver is moved by only a fraction of a wavelength, or the reflecting object moves and the reflected path length changes by only a fraction, or if an obstruction to direct or reflected path occurs (somebody or something obstructs the path) and the path length changes. 


\section{Chapter 3}

\section{Propagation Measurement System}

The reported research was conducted in two phases. In phase 1, a research project conducted collaboratively by Carleton University and Ericsson Canada resulted in transmission loss and shadow models for direct path communication (DPC) derived from measurements made at centre frequency of $2.35 \mathrm{GHz}$, and indirect transmission loss and indirect path loss variations (IPLV) models for indirect path communication (IPC) derived from measurements made at centre frequencies of $18 \mathrm{GHz}$ and 28 GHz. The measurements were conducted in an indoor cluttered environment using a wideband network analyzer provided by Ericsson. In phase 2, narrowband equipment centered at $29.5 \mathrm{GHz}$ were used to study the temporal variation characteristics and distributions for IPC. The rest of this chapter will be divided into two parts; part 1 will describe the wideband radio channel measurement system used in phase 1 and the CW system used in phase 2 of the project will be described in part 2 .

\subsection{Phase 1: Wideband Radio Channel Measurement System}

Propagation measurements are needed to properly design digital wireless systems operating with high data rates in a typical indoor office environment at frequency bands 


\subsection{PHASE 1: WIDEBAND RADIO CHANNEL MEASUREMENT}

SYSTEM

above $6 \mathrm{GHz}$. The propagation characteristics are usually estimated by conducting measurements using the channel sounding technique over radio channels typical of the type of channel associated with the wireless system of interest.

A wideband channel sounder is a system that detects the electromagnetic wave transmitted via a particular communication channel to determine the characteristics of either the channels time-varying impulse response or the time-varying frequency response. The multipath signals are usually referred to as multipath components and their extent in time delay is used to aid in the design of wireless communication systems. Sounders can be deployed in a static or dynamic mode of operation. Static operation refers to the situation where both the transmitter and the receiver are stationary whereas dynamic operation refers to the situation when one or both terminals are in motion. In either case the target, environment, or reflectors can be stationary or in motion. A system that deploys separate antennas or has a separate site for transmission and reception is termed bistatic as shown in Figure 3.1. Whereas, a static system that uses the same antenna for transmission and reception is termed monostatic.

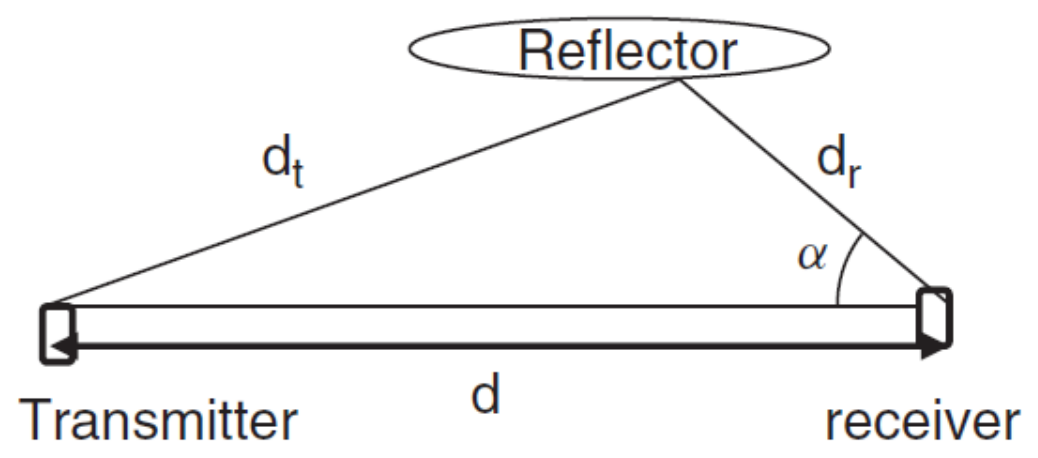

Figure 3.1: Configuration of a bistatic sounder system 


\subsection{PHASE 1: WIDEBAND RADIO CHANNEL MEASUREMENT}

SYSTEM

As opposed to free space, in a typical indoor environment, multipath reflections can be received from objects like walls, furniture, etc..., the received signal is the vector sum of the multipath waves received from all directions, and this vector sum varies in space, as the multipath waves add with different phase relationships in accordance with the lengths of the paths over which they travel or because of the reflection that can also cause a phase shift. If the Tx or/and Rx antennas, or anything in the environment moves, received signal power varies in time as a result of time varying vector addition of the component waves. It is generally accepted that when propagation loss is considered, the effects of such multipath propagation are removed. Then, later in the system design process, account can be made for temporal or spatial variations by adding a received power margin in accordance with known statistical distributions of such power variations. The effects of the multipath-induced power variations can be mitigated and often almost eliminated by averaging over time, space, or frequency. Herein, averaging over frequency, which is new contribution to the field of propagation measurements, have been used. More details are given later in this chapter.

\subsubsection{Wideband Radio Channel Measurement Techniques}

Since a wide sounding bandwidth can be used for the mitigation of multipath effects, using the averaging over frequency technique, in the estimation of transmission loss, and is also needed to estimate delay dispersion, wideband transmission was chosen for the use in channel sounding for this phase.

To gain knowledge of the wideband radio channel, there are two possible techniques to perform the channel sounding. Channel can be measured in time domain 


\subsection{PHASE 1: WIDEBAND RADIO CHANNEL MEASUREMENT}

SYSTEM

measurement that is based on the impulse transmission. The other method is in frequency domain using a frequency sweep technique. In the former technique, a narrow pulse is sent to the channel and the channel impulse response is measured using a digital sampling oscilloscope. The corresponding train of impulses can also be generated using a conventional direct sequence spread spectrum based measurement system with a correlation receiver. In the latter case, a wide frequency band is swept using a set of narrowband signals to measure the insertion loss (IL), known as $S_{21}$ - parameter, which is defined for two port networks as $10 \times \log _{10}\left(P_{R x} / P_{T x}\right)$ where $P_{R x}$ equals the power present at Rx port and $P_{T x}$ equals the power present at Tx port. Theoretically, having a static measurement environment, and occupying an unlimited bandwidth, both techniques end up in the same result. The ideas for the time domain and the frequency domain measurement concepts are presented in Figure 3.2 and Figure 3.3, respectively. The swept frequency sounding was chosen since it can easily be implemented for operation over multiple frequency bands using a commercially-available Vector Network Analyzer (VNA). When a VNA is employed, not only the frequency band to be sounded is selectable, but also the sounding bandwidth. A drawback with using the VNA approach is that time series measurements can only be made at a single frequency, and over very short duration. Thus, frequency dispersion was not studied.

The physical indoor environment during the sounding was kept as static as possible, but no effort was made to control the radio interference from the other RF sources. 
3.1. PHASE 1: WIDEBAND RADIO CHANNEL MEASUREMENT SYSTEM

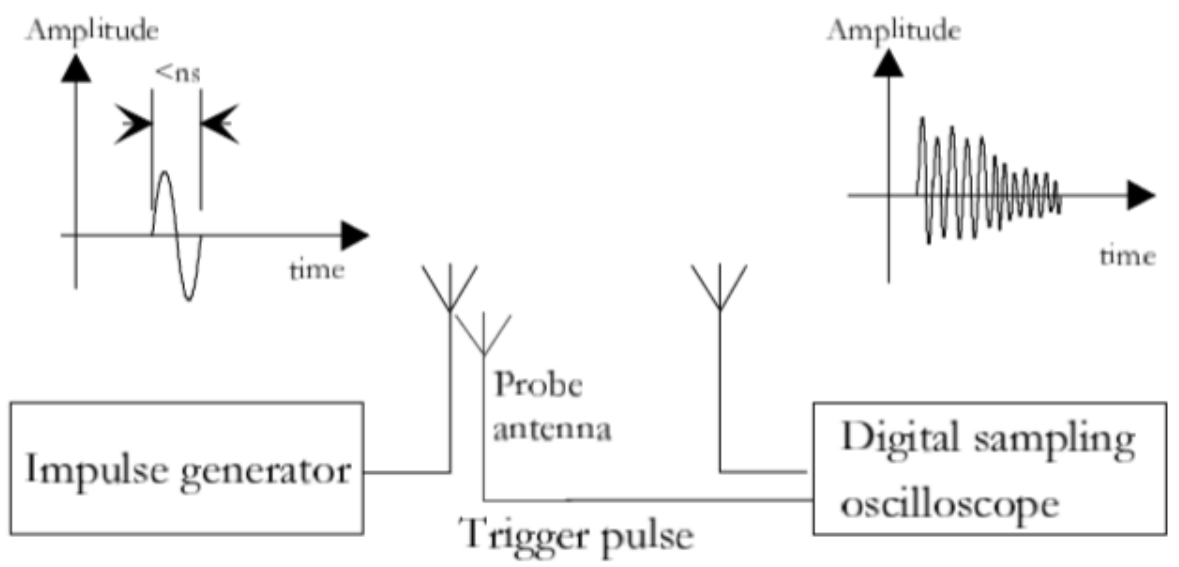

Figure 3.2: Radio channel measurement system based on a pulse transmission

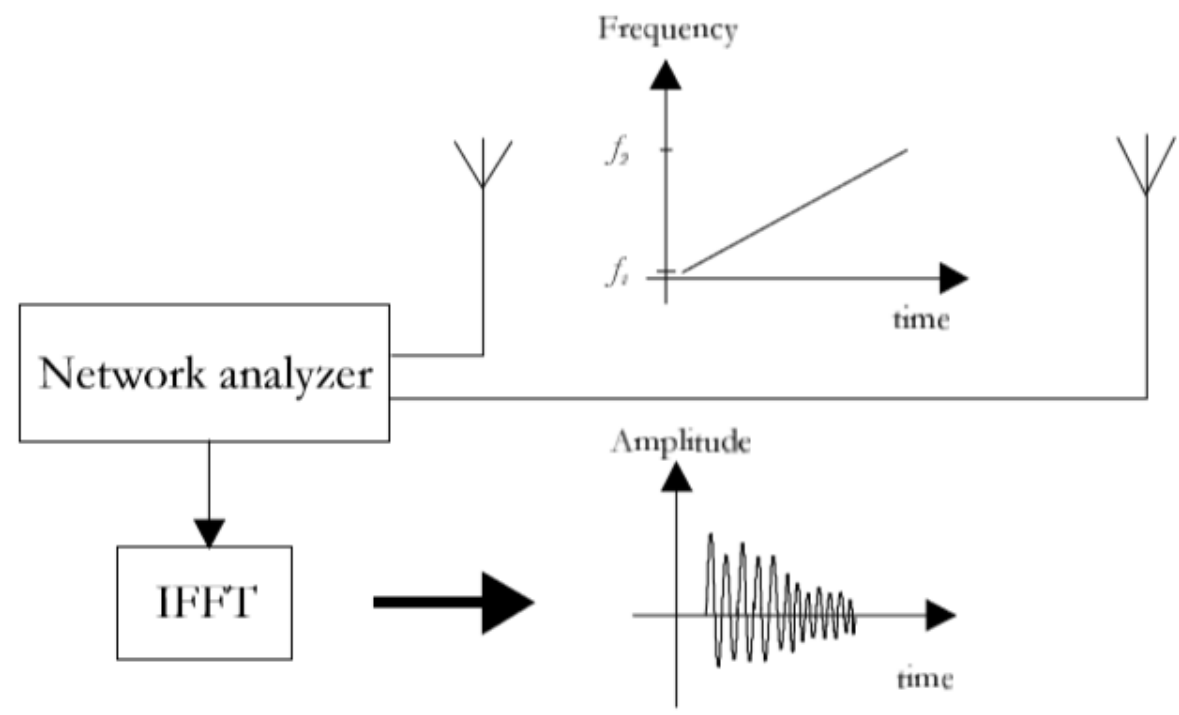

Figure 3.3: Radio channel measurement system based on frequency sweeping 


\subsection{PHASE 1: WIDEBAND RADIO CHANNEL MEASUREMENT}

\subsubsection{Measurement Setup}

The radio channel measurement system consists of a vector network analyzer (Anritsu $37397 \mathrm{C}$ ), sweeping a bandwidth of $500 \mathrm{MHz}$ in $1.25 \mathrm{MHz}$ steps. The power at the Tx port output was set to $-7 \mathrm{dBm}$, and this port was connected to the Tx antenna using $5 \mathrm{~m}$ of UFB142A-0 low loss transmission line. The receive antenna was connected to the receive port of the VNA using $3 \mathrm{~m}$ of EZFlex low loss transmission line. A pre-amplifier (Agilent 83017A) that had gains of $0 \mathrm{~dB}$ at $2 \mathrm{GHz}, 21.5 \mathrm{~dB}$ at $18 \mathrm{GHz}$, and $27 \mathrm{~dB}$ at $28 \mathrm{GHz}$ was inserted in the receive transmission line as close to the receive antenna as possible. A set of antennas were used to conduct the measurements, in which, an appropriate pair of antennas was chosen based on the scenario to be experimented. AINFO 2-18 GHz dual-polarized horn, AINFO 18-40 GHz dual-polarized horn, and 2-18 GHz omni directional biconical are the antennas being used in the measurements. The sweep time is automatically adjusted by the analyzer, depending on the bandwidth and the number of the measured frequency points within the sweeping band. The $500 \mathrm{MHz}$ bandwidth was chosen after considering the multipath delay, antenna bandwidth, and interference avoidance. The block diagram of the measurement setup is presented in Figure 3.4 where also the main features of the post-processing procedure are presented. 


\subsection{PHASE 1: WIDEBAND RADIO CHANNEL MEASUREMENT}

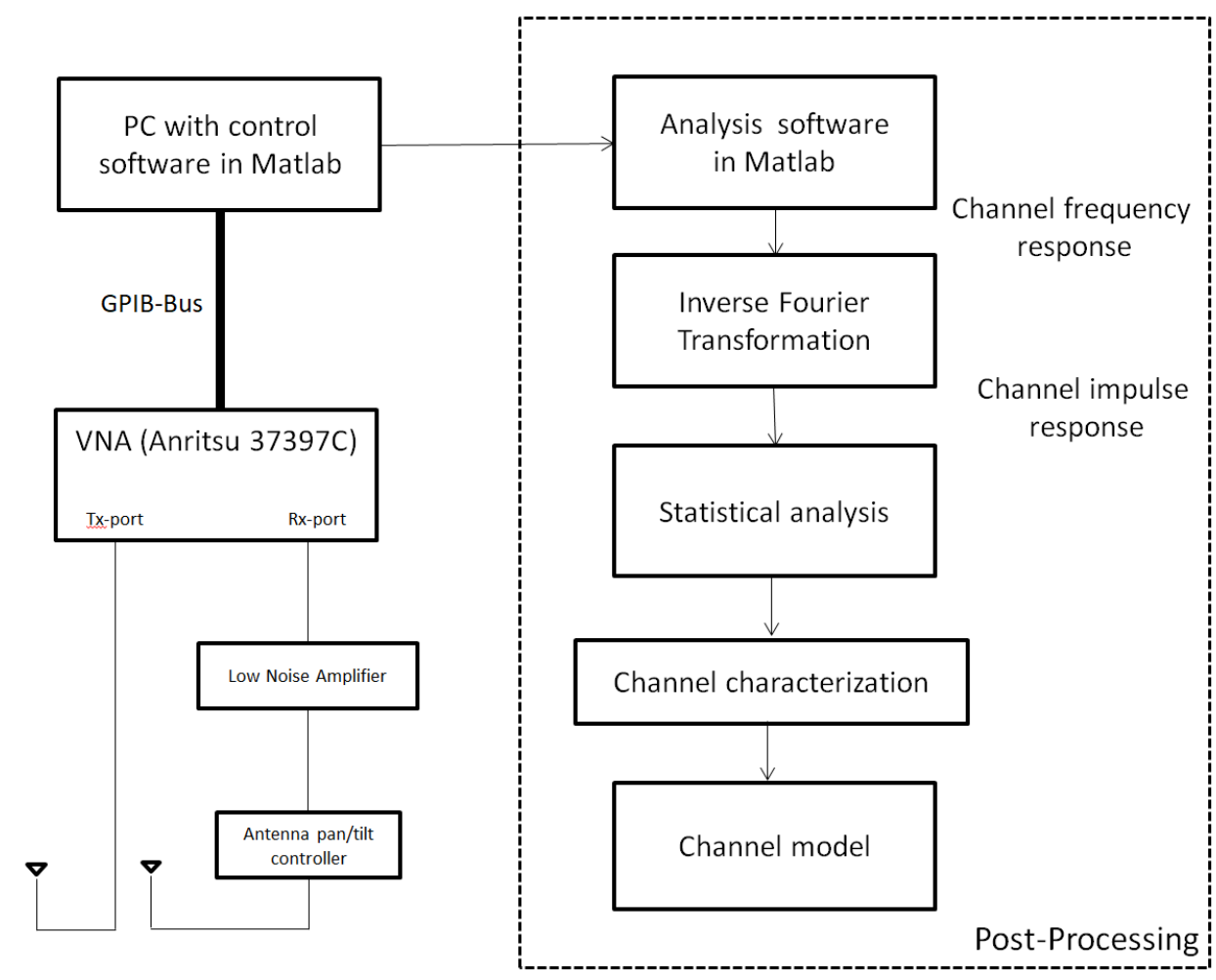

Figure 3.4: Block diagram of the radio channel measurement system

One of the typical uses for VNAs is to measure IL of the device under test (DUT). However, the VNA requires a calibration with the same transmission lines and adapters as will be used in the measurements. This can be done by connecting its Tx subsystem directly to its Rx subsystem, and allowing the VNA to compensate electronically for those characteristics of the transmission lines so the displayed results of IL and phase shift are zeroed and linearized, respectively. Since the amplifier is isolated in reverse direction, it has to be removed from the setup for the time of calibration. Also, unless anechoic chamber is used, the antennas need to be removed at the time of calibration, however, transmission lines were good enough that moving to anechoic chamber for the calibration was considered to only provide incremental 


\subsection{PHASE 1: WIDEBAND RADIO CHANNEL MEASUREMENT}

SYSTEM

improvement, at significant expenditure of resources. Therefore, the calibration was done without the amplifier and antennas, later in the post-processing, the characteristics specified by their manufacturers were accounted for in equations relating measured insertion loss to transmission loss. Figure 3.5 shows an insertion loss measurement that was made at a centre frequency of $2.35 \mathrm{GHz}$, a bandwidth of $500 \mathrm{MHz}$ and sample spacing in frequency of $1.25 \mathrm{MHz}$ via a back-to-back connection. As can be seen, the insertion loss magnitude is almost zero over all the specified bandwidth, and the phase shift is linear.
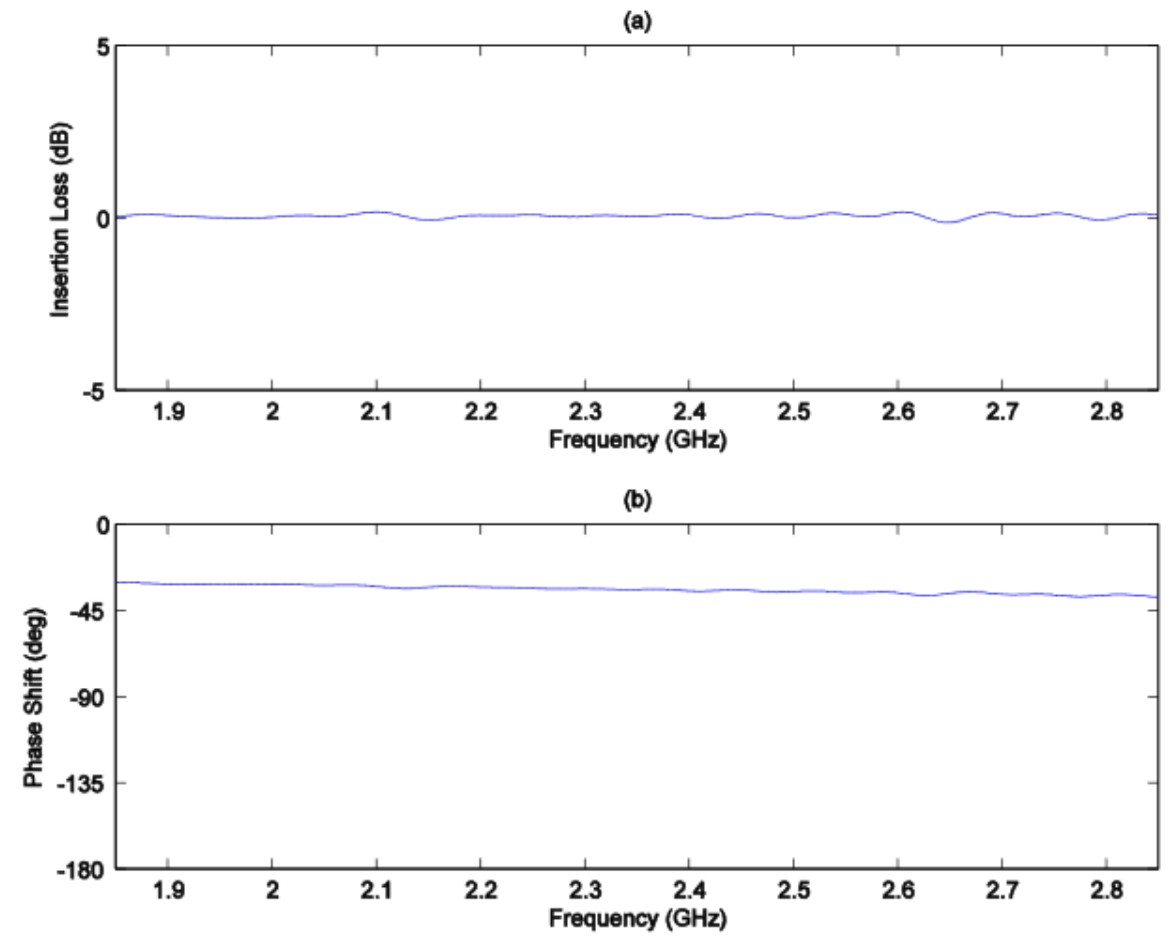

Figure 3.5: Back-to-Back VNA-based channel sounder system insertion loss at $2.35 \mathrm{GHz}$ (a) magnitude, (b) phase

In using VNA based channel sounders, it is important to ensure that the frequency 


\subsection{PHASE 1: WIDEBAND RADIO CHANNEL MEASUREMENT} SYSTEM

step size is small enough that its reciprocal is greater than the longest multipath propagation delay expected on the channel. It is also important to use a total bandwidth that yield acceptable resolution in multipath delay. After considering these factors as well as measurement time and storage requirements, 400 samples spaced $1.25 \mathrm{MHz}$ apart was chosen for this work. Therefore, the maximum multipath delay that can be measured based on that is equal to $0.8 \mu \mathrm{s}$ corresponding to $240 \mathrm{~m}$ propagation distance. Based on consideration of the dimensions and layout of the measurement environment, this is considered to be good enough. The delay resolution resulting from $500 \mathrm{MHz}$ total bandwidth is $2 \mathrm{~ns}$. This is typical of maximum delay resolutions for radio channel sounders used by wireless system and cellular radio propagation research organizations worldwide.

To measure the path loss, insertion loss values are transformed into transmission loss after accounting for the transmit power, antenna gains, power amplifier gain, and line losses. Hence, the received power in $\mathrm{dB}$ is given as

$$
\mathrm{P}_{R}=\mathrm{P}_{T}+\mathrm{G}_{T}+\mathrm{G}_{R}-\mathrm{L}_{T}-\mathrm{L}_{L_{T}}-\mathrm{L}_{L_{R}}-\mathrm{IL}
$$

where $\mathrm{P}_{T}$ is the VNA Tx transmit power. $\mathrm{G}_{T}$ and $\mathrm{G}_{R}$ represent $\mathrm{Tx}$ and $\mathrm{Rx}$ antenna gains respectively, $\mathrm{L}_{T}$ is the transmission loss, and IL represents the measured insertion loss, $L_{L_{T}}$ and $L_{L_{R}}$ are the transmit and receive subsystem line losses, respectively. One can write the measured finite-bandwidth estimate of the transfer function $H(f)$

$$
\mathrm{H}(f)=10^{(\mathrm{IL}(f) / 10)} \cos \Phi(f)+j 10^{(\mathrm{IL}(f) / 10)} \sin \Phi(f)
$$

where $\Phi(f)$ is the measured phase shift, the corresponding finite-bandwidth impulse 


\subsection{PHASE 1: WIDEBAND RADIO CHANNEL MEASUREMENT} SYSTEM

response estimate is then given by the inverse Fourier Transform (IFT) of $H(f)$

$$
h(\tau)=\int_{-\infty}^{+\infty} H(f) e^{+j 2 \pi f \tau} d f
$$

where $\tau$ represents propagation delay. To mitigate spectral leakage, windowing in the frequency domain was applied. A Hanning window was chosen for this application after considering different window shapes and their effects on the results. Figure 3.6 shows the impulse response estimate IRE corresponding to the IL measurement shown in Figure 3.5.

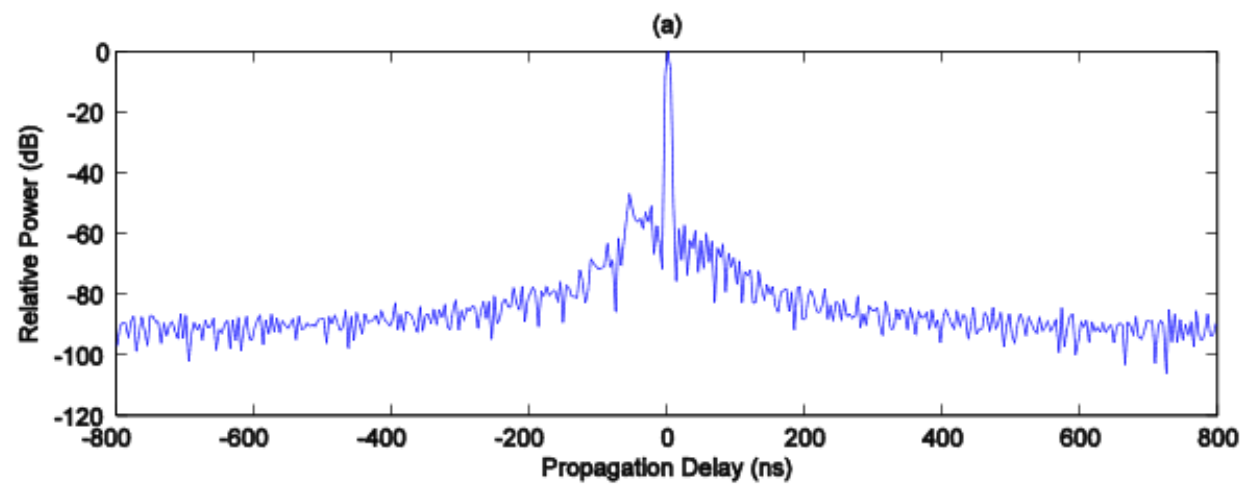

(b)

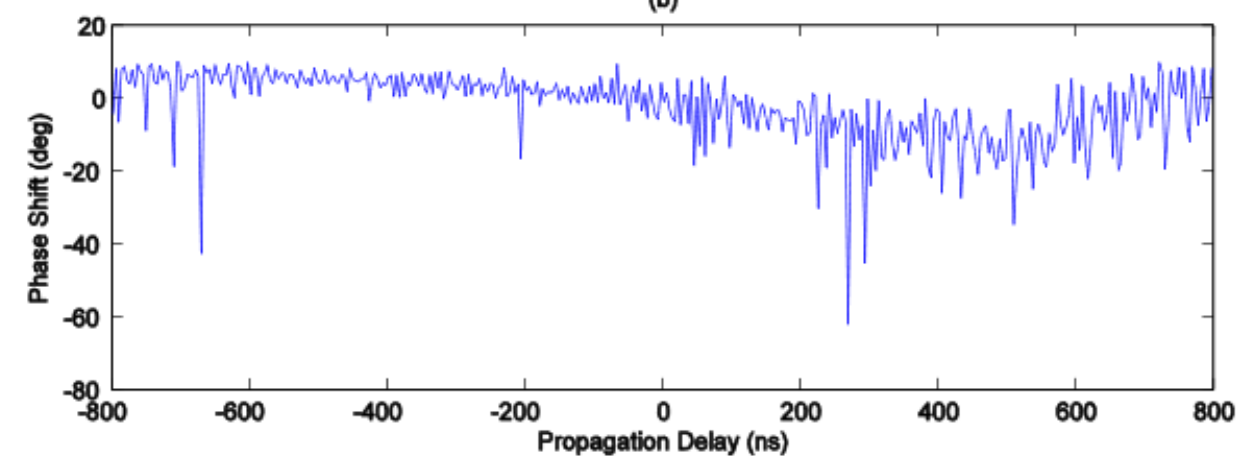

Figure 3.6: Back-to-Back VNA-based channel sounder system impulse response estimate at centre frequency $2.35 \mathrm{GHz}$

As it can be seen from the figure, the impulse response peak is slightly delayed 


\subsection{PHASE 1: WIDEBAND RADIO CHANNEL MEASUREMENT}

SYSTEM

reflecting the delay in the transmission lines. Minor reflections appear after the impulse, due to adapter and transmission lines small mismatch, followed by a relatively constant noise floor at a relative power of about $-87 \mathrm{~dB}$. It is a typical procedure to plot a circularly shifted and truncated version of the IRE as a function of excess delay, i.e., delays of multipath echos relative to the earliest received signal at a point where the back-to-back IRE begins to rise from the noise floor to the IRE peak. The subplots of Figure 3.7 show circularly shifted back-to-back IREs for 2.35, 18, and 28 $\mathrm{GHz}$.

The subplots show that the noise and reflections due to system imperfections have relative powers less than $-45 \mathrm{~dB}$ in all cases. These results are when VNA Tx power set to its maximum of $-7 \mathrm{dBm}$. The maximum width of IREs shown in the same figure at $-20 \mathrm{~dB}$ relative power is $5 \mathrm{~ns}$. These characteristics are considered excellent for any radio channel sounding system. 


\subsection{PHASE 1: WIDEBAND RADIO CHANNEL MEASUREMENT}
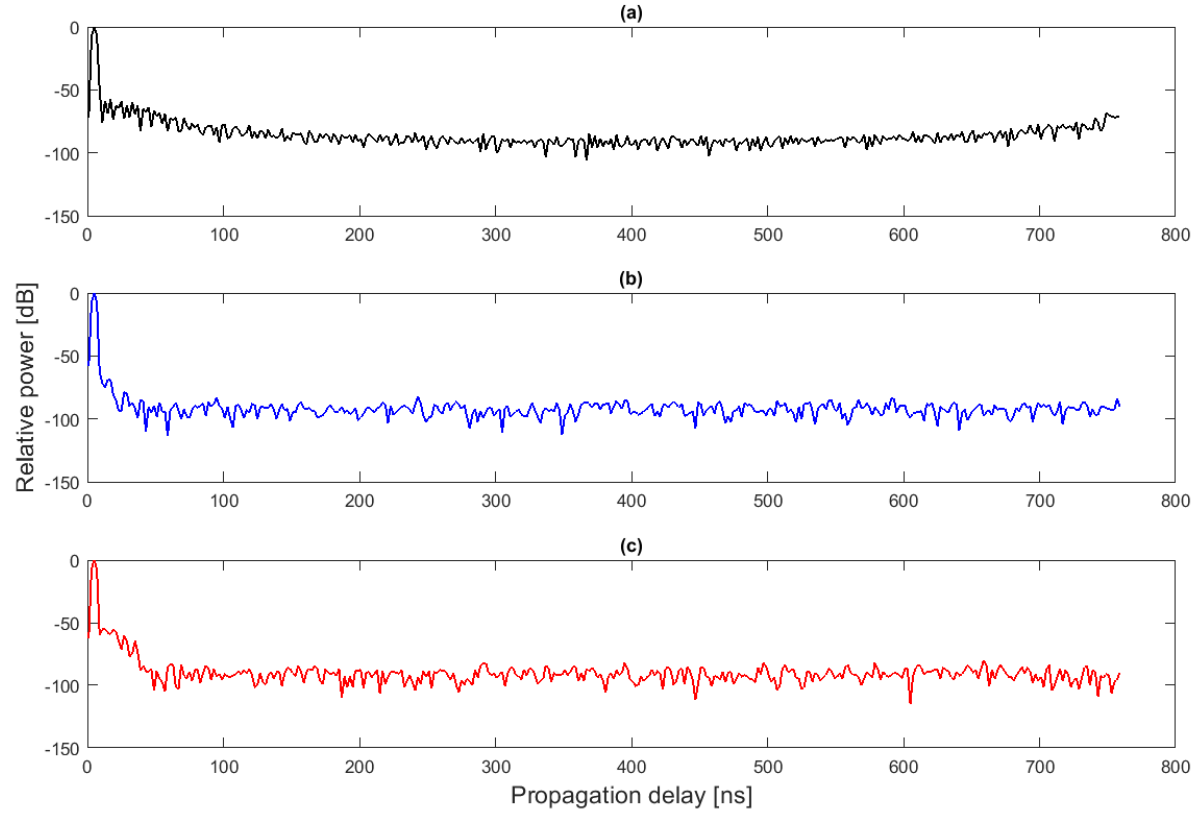

Figure 3.7: Back-to-Back IREs: (a) $2.35 \mathrm{GHz}$, (b) $18 \mathrm{GHz}$, (c) $28 \mathrm{GHz}$

Having accurate IRE is crucial to estimate the delay dispersion on measured channels. One can define delay dispersion as the maximum excess delay in a measured IRE. However, this definition can only apply when all multipath echos have equal power level. In practice, the echos with the longest delay have lower power levels, and therefore root mean square (RMS) delay spread, or the power weighted variance of excess delays, is estimated instead. In order to get correct RMS delay spread, only valid multipath echos should be considered, and this can be done by noise thresholding, i.e. nulling the noise samples of the channel having values less than a threshold. In order to apply noise thresholding, noise characteristics need to be known first, one method is to consider the last $n$ samples (50 or so) in measured complex IREs. It can be assumed that the noise components of the in-phase and quadrature components are independent Gaussian distributed with zero mean and $\sigma^{2}$ variance, giving 


\subsection{PHASE 1: WIDEBAND RADIO CHANNEL MEASUREMENT} SYSTEM

a Rayleigh distributed envelope with a PDF expressed by:

$$
P(r)=\frac{r}{\sigma^{2}} e^{\frac{-r^{2}}{2 \sigma^{2}}}
$$

where $\sigma$ is the standard deviation of the envelope of IRE $n$ sample values with the largest excess delay. To establish a noise threshold level $R$ for the detection of the multipath components, a certain probability of error is allowed where an error occurs if the threshold is too low and a noise peak is detected as a signal or if the threshold is too high and the signal is missed. These situations are referred to as false alarm or a miss respectively. For a Rayleigh distribution the median and the mean are given by $\sigma_{m}=1.1774 \sigma$ and $\mu=1.2533 \sigma$ respectively. For a threshold level equal to $R$, a false alarm occurs if the magnitude of noise exceeds $R$. Thus the probability of a false alarm per sample $P_{f}$ is given by

$$
P(f)=\operatorname{prob}[r>R]=\int_{R}^{\infty} P(r) d r=\exp \left(-\frac{R^{2}}{2 \sigma^{2}}\right) .
$$

The value of the threshold $R$ is chosen to meet a certain acceptable error rate. Taking logarithms, at the median $|r|_{50}$, Eq (3.5) can be rearranged to give

$$
\sigma=\sqrt{\frac{-|r|_{50}^{2}}{2 \ln (0.5)}}=.849|r|_{50}
$$

Thus, if the median of the last $n$ samples is estimated, $\sigma$ can also be estimated. Then for any desired PDF, the envelop voltage $|r|_{t h}$ can be calculated from Eq (3.5) as

$$
|r|_{t h}=\sqrt{2 \sigma^{2}|\ln (\mathrm{PFA})|}
$$

where PFA is the probability of false alarm. Once a value of PFA has been decided, a 


\subsection{PHASE 1: WIDEBAND RADIO CHANNEL MEASUREMENT}

SYSTEM

noise rejection threshold can be set using Eq (3.7) to find $|r|_{t h}$, such that if any sample have voltages below this threshold, they are set to zero so they have no influence on the estimation of the dispersion. For the truncated IREs reported herein, which have 380 samples each, this PFA would be $1 / 760$, or $1.3 \times 10^{-3}$, and the noise rejection threshold would be set at $|r|_{t h}=3.6418 \sigma=3.0930|r|_{50}$. Measures of delay dispersion can be estimated following such thresholding.

\subsubsection{Mitigation of Multipath Interference}

In a far-field multipath radio propagation environment, single frequency radio waves interfere, and add vectorially at any point in space, creating a resultant with power that can be considerably different than that predicted using Friis' equation for free space propagation. For such environments, however, it is necessary to develop models for average propagation loss as a function of transmitter-receiver ( $\mathrm{Tx}-\mathrm{Rx})$ separation distance that can be used for system design purposes. To provide data for model development, a CW signal is often transmitted and the resulting received signal power is measured as a function of distance from the transmitter. In order to mitigate the aforementioned multipath interference to yield the average received power in a local area of about 1 wavelength in radius at any given distance, the receive antenna should be rotated, or moved randomly over the area of a disc while recording received powers, and the recorded results should be averaged.

At frequencies in the range of $2 \mathrm{GHz}$ and lower, with omnidirectional antennas, it is relatively easy to devise mechanical devices that will rotate, or otherwise move a receive antenna automatically to measure a sufficient number of received powers in local area of radius 1 wavelength for averaging. However, at higher frequencies 


\subsection{PHASE 1: WIDEBAND RADIO CHANNEL MEASUREMENT}

SYSTEM

where wavelengths are shorter, and where directional receive antennas are of interest, the design of such devices for motion of the antenna becomes considerably more difficult as a result of the constraints that the receive antenna must not be moved so far as to change the average propagation loss, and its pointing direction must remain constant. A means for averaging other than by recording powers at different locations throughout a local area is therefore of great interest.

If one considers the physical process involved in multipath propagation, it can be noted that at a specific frequency $\left(F_{a}\right)$, and any given point in space (say Pt. A), the distances travelled by many of the multipath waves and results of their interactions with objects along their paths are different, giving them different amplitudes and different phases. The sum of these waves is therefore the vector sum of $N$ phasors with different amplitudes and different phases constrained to be on the interval $(0,2 \pi]$. At any different closely-spaced point in space, (say Pt. B) at the same frequency $\left(F_{a}\right)$, the same $N$ waves are received, but their amplitudes and phases are different, with the differences being a linear function of the differences in distance the waves travelled to arrive at Pt. B rather than Pt. A. As at Pt. A, the sum of the waves at Pt. B and $\left(F_{a}\right)$ is the vector sum of $N$ phasors, but the amplitude and phase of each phasor are incrementally different than at Pt. A, with the phases still constrained to be on the interval $(0,2 \pi]$.

At the same point in space (i.e. Pt. A) but at any different closely spaced frequency (say, $\left(F_{b}\right)$ ), the same $N$ waves are received, but their amplitudes and phases are different, with the differences being a linear function of the difference between $\left(F_{a}\right)$ and $\left(F_{b}\right)$. As at Pt. A and $\left(F_{a}\right)$, the sum of the waves at Pt. A and $\left(F_{b}\right)$ is the vector sum of $N$ phasors, but the amplitude and phase of each phasor are incrementally 


\subsection{PHASE 1: WIDEBAND RADIO CHANNEL MEASUREMENT}

different than at $\left(F_{a}\right)$.

It can be noted that the set of vector sums of a group of $N$ waves at different points in space and the same frequency has very similar characteristics to those of a set of vector sums of the same group of $N$ waves at different frequencies but the same point in space, with both groups of waves having approximately the same amplitudes over a limited frequency range and incremental distances, and phases constrained to be in the interval $(0,2 \pi]$. It is conjectured, therefore, that if $N$ is sufficiently large, there is significant probability that averaging the powers corresponding to the vector sums of the $N$ waves at different points in space over a limited range of incremental distances, but at the same frequency should give a result that is close to that obtained by averaging the vector sums of the same waves at different frequencies over a limited bandwidth, but at the same point in space.

Based on the foregoing, it is predicted that averaging received powers over a limited bandwidth at a given distance from a Tx should be as effective in mitigating multipath interference as the commonly-used averaging over a local area centred at the same distance from the Tx, in order to derive data that can be used in the development of models for average propagation loss as a function of Tx-Rx separation. A set of propagation experiments were therefore conducted to investigate this prediction. These are reported in Section 4.1.1 of this thesis. Results show that for Tx-Rx separation greater than $1 \mathrm{~m}$, frequency averaged transmission loss models for an empty room very closely follow results from friis' equation for free space propagation. Averaging powers over $500 \mathrm{MHz}$ bandwidth centred on any of the seventh centre frequencies where measurements were conducted was therefore effected during all transmission loss analyses reported in this thesis. 


\subsection{PHASE 1: WIDEBAND RADIO CHANNEL MEASUREMENT}

SYSTEM

\subsubsection{Noise Floor of the VNA Sounding System}

In radio systems, measurements can get corrupted by noise when received power falls below what is known as the noise floor of the system. The Anritsu $37397 \mathrm{C}$ VNA that was used for this work has a built in capability to reduce the Tx subsystem output power on command, or to attenuate the received signal on command. The ranges overwhich this can be done are $20 \mathrm{~dB}$ and $60 \mathrm{~dB}$ respectively in steps of $1 \mathrm{~dB}$. The noise floor was estimated by using the following steps:

1. Adjusting the VNA Tx subsystem to output full power $(-7 \mathrm{~dB})$.

2. Using the attenuation function on the VNA, attenuating the received signal in steps of $5 \mathrm{~dB}$.

3. before the first, and after each subsequent attenuation step, a VNA sweep to record 400 samples across $500 \mathrm{MHz}$ was made in the frequency band of interest.

4. The resulting data files were analyzed and reported as below.

Figure 3.9 shows the total power in back-to-back IREs after thresholding them for noise rejection at $-20 \mathrm{~dB}$, for the $500 \mathrm{MHz}$ bandwidths centered at $2.35 \mathrm{GHz}$ as a function of VNA receive subsystem input power. 


\subsection{PHASE 1: WIDEBAND RADIO CHANNEL MEASUREMENT}

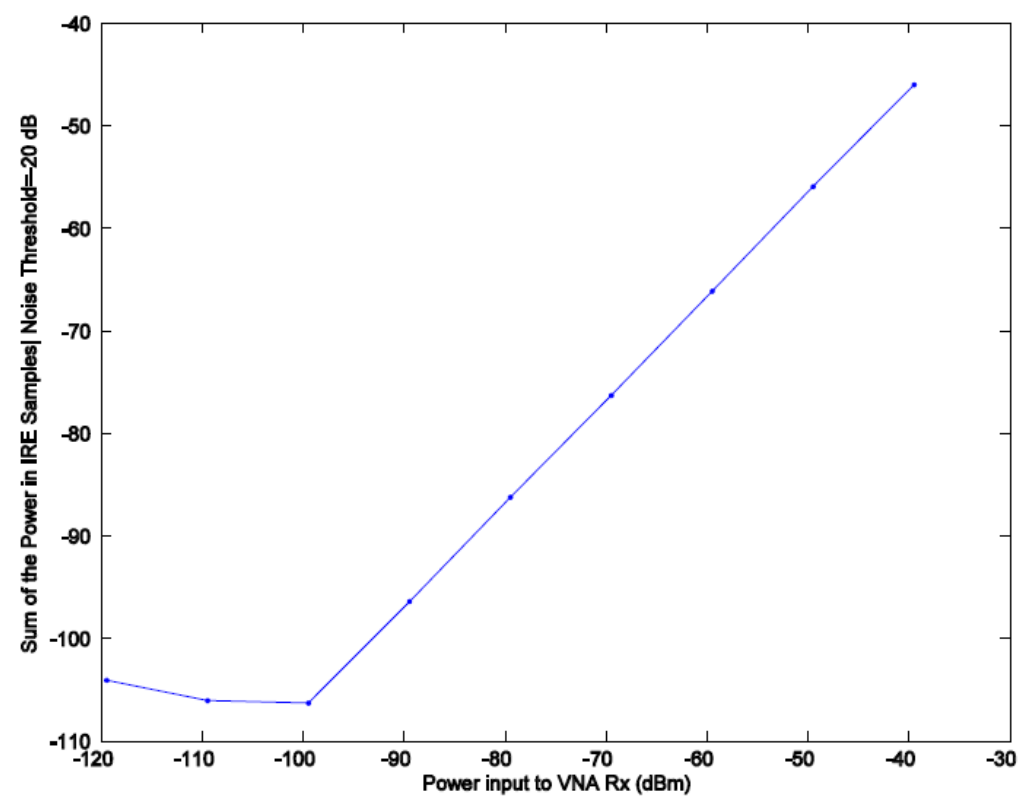

Figure 3.8: The power in IREs estimated from back-to-back measurements as a function of VNA receive subsystem input power in the $2 \mathrm{GHz}$ band

As it can be seen from Figure 3.9, the powers no longer decreased with added Rx subsystem input attenuation after the power received by the VNA receive subsystem dropped below $-100 \mathrm{dBm}$. This was taken as the system noise floor in the $2 \mathrm{GHz}$ band. It is believed that the system contains more noise at lower frequencies. Thus, when analyzing data for other frequency bands, the system noise floor was assumed to be $-70 \mathrm{dBm}$.

\subsubsection{Receive Antenna Directivity and Control of Receive Antenna Point- ing Direction}

As one of the stated objectives in this thesis is to determine the viability of IPC systems, therefore, the channel sounding system used for this study must be able to distinguish direction of arrival. Using 3-D antenna arrays at both the channel 


\subsection{PHASE 1: WIDEBAND RADIO CHANNEL MEASUREMENT}

SYSTEM

sounder transmitter and receiver is the best way to achieve it, then use one of the high-resolution signal processing algorithms, such as MUSIC, ESPRIT, and SAGE to post process measured data. Another simpler method, which was used herein, is to steer a wideband horn Rx antenna mechanically. Two different horn antennas were necessary at the Rx to cover the three frequency bands. These included a 2-18 GHz dual polarized horn, and an 18-40 GHz dual polarized horn. Listed in Table 3.1 are the gain and beamwidths of these antennas. At each measurement location, these horns were mounted on a remotely-controllable pan-tilt unit (PTU), and scanned azimuth and elevation. All measurements were done in each band before moving to the next band.

Table 3.1: Antenna Specifications

\begin{tabular}{|c||c|c|c|c|c|}
\hline \begin{tabular}{|} 
Frequency \\
GHz
\end{tabular} & Antenna & $\begin{array}{l}\text { Gain-V } \\
(\mathrm{dBi})\end{array}$ & $\begin{array}{l}\text { Gain-H } \\
(\mathrm{dBi})\end{array}$ & $\begin{array}{l}\mathrm{BW}-\mathrm{H} \\
(\mathrm{deg})\end{array}$ & $\begin{array}{l}\text { BW-E } \\
(\mathrm{deg})\end{array}$ \\
\hline 28 & Ainfo 18-40 Horn & 14 & 13 & 31 & 35 \\
\hline 28 & Ainfo 2-30 Biconical & 6 & - & - & - \\
\hline 18 & Ainfo 18-40 Horn & 11 & 11 & 44 & 51 \\
\hline 18 & Ainfo 2-30 Biconical & 4.5 & - & - & - \\
\hline 2.35 & Ainfo 2-18 Horn & 9 & 9 & 61 & 59 \\
\hline 2.35 & Ainfo 2-30 Biconical & -3 & - & - & - \\
\hline
\end{tabular}

The PTU and the VNA were both controlled over a general purpose interface bus (GPIB) to:

1. Recall VNA calibration states for each frequency band 
2. Adjust the pointing direction of the RX antenna

3. Initiate data recording

4. Store recorded data for 1 VNA sweep, which included 400 data samples, spaced by $1.25 \mathrm{MHz}$

5. Save recorded data in a Matlab file

Typically, a data recording session involves ten steps, as follows:

Step 1: Recall the VNA calibration data for the $500 \mathrm{MHz}$ bandwidth centered at the frequency targeted for measurement.

Step 2: Adjust the pointing direction of the Rx horn to the steering position with azimuth angle $A Z=-180$ and elevation angle $E L=-60$.

Step 3: Record a single sweep of VNA insertion loss IL amplitude and phase data and save data in "ILoss" array.

Step 4: Repeat step 3 for the desired elevation angle set.

Step 5: Repeat step 3 and 4 for the desired elevation angle set.

\subsection{Phase II: Single Tone Measurement System}

Measurements of envelop fading statistics is one of the main popular applications of continuous wave CW systems. A CW system transmits a continuous wave signal $S_{T}(t)$ at an angular frequency, $\omega_{c}=2 \pi f_{c} \mathrm{rad} / s$ and receives a signal $S_{R}(t)$, which consists of $N$ echoes 


$$
\begin{gathered}
S_{T}(t)=\cos \left(2 \pi f_{c} t\right) \\
S_{R}(t)=\sum_{n=1}^{N} A_{n}(t) \cos \left[2 \pi f_{c}\left(t-\tau_{n}\right)\right]
\end{gathered}
$$

where $A_{n}(t)$, and $\tau_{n}$, are the amplitude and the time delay of the $n_{t h}$ echo or multipath component, respectively. The single tone measurements were made using continuous wave equipment that was designed and built in a joint project between the Communication Research Centre (CRC) and Carleton University in the 1990s. The system consists of a transmitter, a receiver, and a data collection system.

\subsubsection{CW Transmitter}

The transmitter unit consists of two identical Gunn oscillators each feeding a three inche length of a waveguide, and a linearly polarized antenna. Figure 3.10 shows the basic configuration of the transmitter. The waveguide of one of the oscillators has 90 degree twist to provide a vertical E-field polarization in one antenna and a horizontal E-field polarization in the other antenna. Both oscillators have been adjusted to operate at a frequency of $29.5 \mathrm{GHz}$ after the warm-up period of about 30 minutes. The antenna of the transmitter that is used in the envelop fading measurements is horn antennas with a maximum gain of $17 \mathrm{dBi}$. There is a dummy load on each transmitter to limit the output of the transmitter so it is safe for indoor use, applied in this work, by providing $25-30 \mathrm{~dB}$ attenuation resulting in a $20 \mathrm{dBm}$ output power. Thus, the transmitter will produce an EIRP of $37 \mathrm{dBm}$ ( $20 \mathrm{dBm}$ output power +17 dBi antenna gain). Note that only the vertical polarization antenna was used for the 
work reported in this phase. The antenna has a beamwidth of 26 degrees.

\subsubsection{CW Receiver}

The receiver unit consists of a 26.5 to $40 \mathrm{GHz}, 11$ degree, $24 \mathrm{~dB}$ gain horn antenna, Power supply input, IF loop. Figure 3.11 shows the receiver configuration. A power supply of between 10 to 17 volts at less than 0.7 amps was required for proper functioning of the receiver unit. The receiver uses VCOs to perform signal down-conversions to a $2_{n d}$ stage IF of $160 \mathrm{MHz}$, where the output feeds a logarithmic amplifier. The latter amplifier outputs a dc voltage that is proportional to the input signal power. This de is sampled using a National Instruments data acquisition device (NI myDAQ) and the sampled voltages are stored on a personal computer PC for later comparison with a received power vs log amplitude voltage calibration relationship.

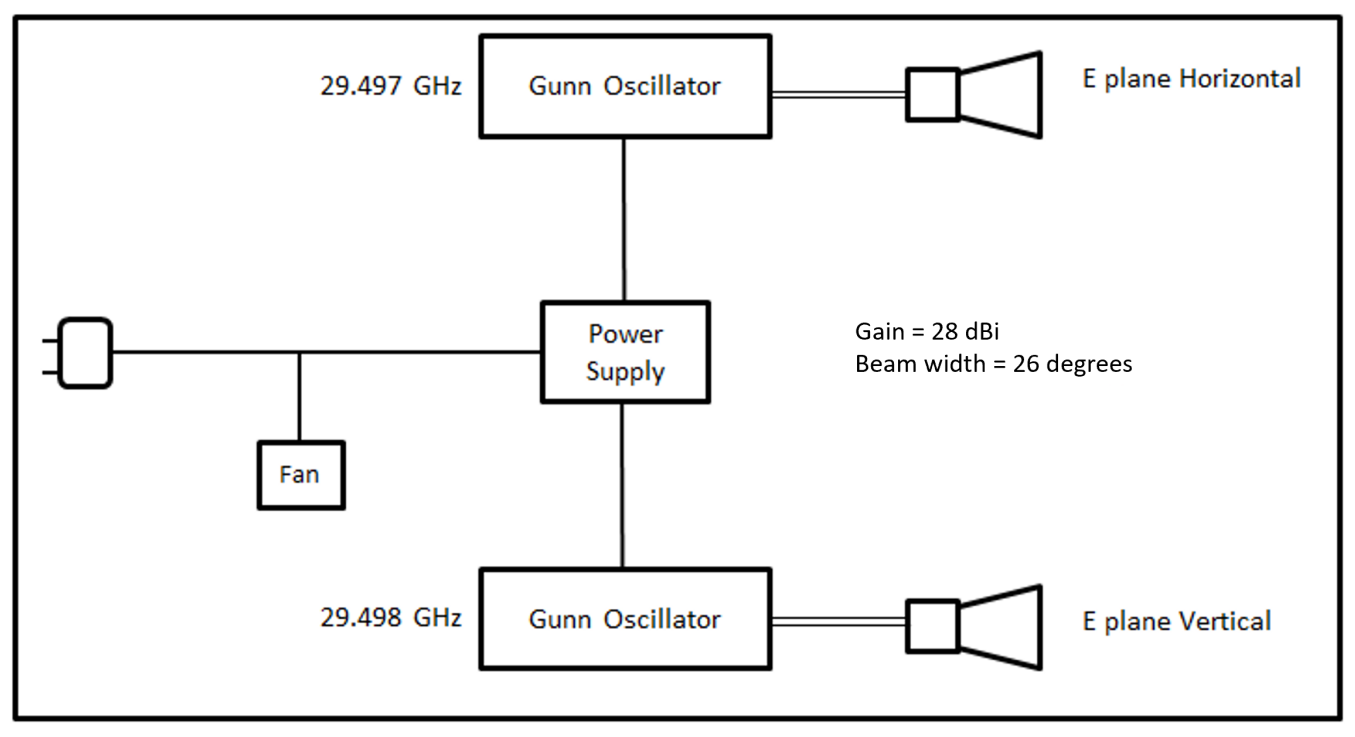

Figure 3.9: Block diagram of the $\mathrm{CW}$ transmitter 


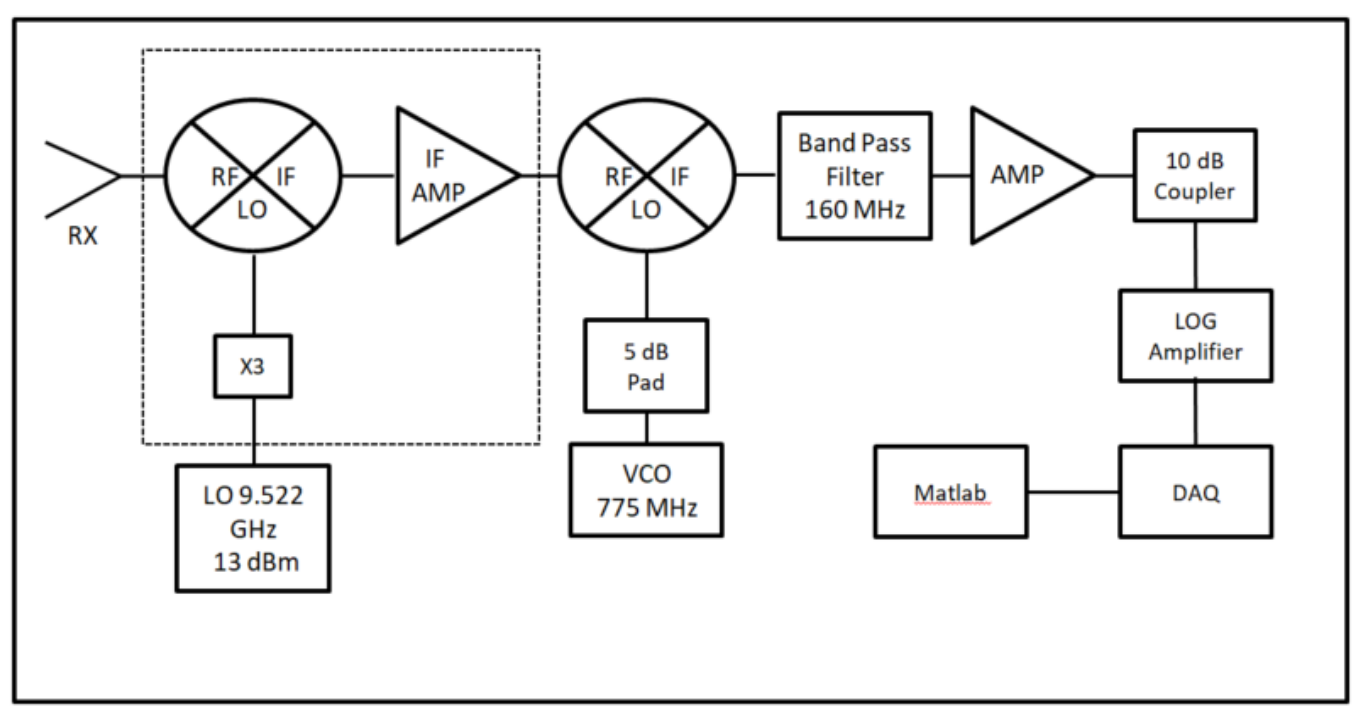

Figure 3.10: Block diagram of the CW receiver

\subsubsection{Data Collection System}

The data collection system consists of a data acquisition device (DAQ) and a computer with programmable software. The DAQ hardware acts as the interface between a computer and the analog signal that is coming from the receiver unit. The three key components of a DAQ device used for measuring a signal are the signal conditioning circuitry, analog-to-digital converter (ADC), and computer bus. The programmable software controls the operation of the DAQ device and is used for processing, visualizing, and storing measurement data. 


\section{Chapter 4}

\section{Propagation Measurements and Modelling for Indoor Channels}

\subsection{Phenomenological Measurements}

As an initial step before conducting channel modelling for cluttered office spaces, measurements of propagation phenomena at frequencies in the 2-30 $\mathrm{GHz}$ range were made, and results are reported in this chapter. The phenomena studied are believed to be enabling phenomena as they are essential for any IPC system to operate. These measurements were carried out in the daytime in a small study room within a hightech complex in Ottawa.

The room in which the measurements were conducted had dimensions of $5 \mathrm{~m}$

long $\times 3.6 \mathrm{~m}$ wide $\times 4 \mathrm{~m}$ high, with a concrete floor, a concrete ceiling at the $4 \mathrm{~m}$ height, and walls made of plasterboard mounted on galvanized steel studs separated from each other by $48 \mathrm{~cm}$. The room had a suspended ceiling at a height of $2.75 \mathrm{~m}$, comprised of soft tiles hung within a metal grid, which also supported air conditioning vents and light fixtures.

The reported measurements were made at centre frequencies of $2.35 \mathrm{GHz}, 5.2$ 
$\mathrm{GHz}, 10 \mathrm{GHz}, 15 \mathrm{GHz}, 22 \mathrm{GHz}$, and $28 \mathrm{GHz}$.

\subsubsection{Empty Room Measurements}

\section{Line of Sight Propagation Loss}

Line of sight propagation loss measurements in an empty room can be used as proof of measurement system performance when compared with already known results from a trusted measurement system, or when compared with Friis' equation after averaging over frequency to eliminate multipath effects, as explained in Chapter 3. This section, therefore, reports results of propagation loss between two antennas as a function of distance, averaged over $500 \mathrm{MHz}$ bandwidth for multipath mitigation and converted to received power using Eq (3.1).

As shown in Figure 4.1, the measurements were taken in an empty room; the Rx horn antenna was moved manually along the centreline in steps of $5 \mathrm{~cm}$ from the Tx antenna which was an omnidirectional antenna. At each location, IL was measured in each of the selected frequency bands within the operating range of the receive horn antenna being used. Figure 4.2 shows results. Each subplot in the figure shows results from applying Friis' equation, results from the experiments, and ray-tracing results $^{1}$. The ray-tracing results are single frequency results and therefore show the spatial variations that result from multipath interference, whereas the averaging over frequency in the measured results has effectively eliminated multipath effects, and Friis' equation is for the absence of multipath propagation. The comparisons are good in all frequency bands, except at short Tx-to-Rx antenna separations. It can

\footnotetext{
${ }^{1}$ The Ray-Tracing in this thesis is simulated using a MATLAB code written to simulate the physical phenomena in a small empty room with smooth, plane walls, with the same dimensions of the actual room where measurements were made, and the walls and floor were assigned the properties of those in the actual room.
} 
also be seen that the general trend is for the errors at short separations to get larger as frequency increases. The errors are believed to be due to antenna pointing problems, where the Rx horn antennas were not pointing directly at the Tx biconical antenna. However, since the beam patterns of the horn antennas broaden as the distance between Tx and Rx antennas increases, the pointing errors start to diminish and the theoretical and measured results converge in all frequency bands.

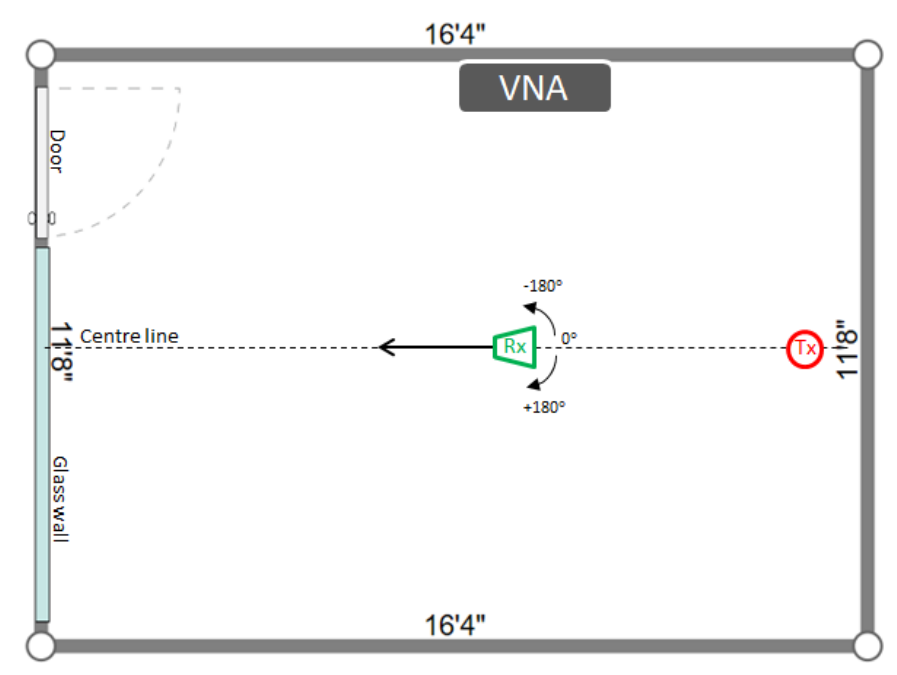

Figure 4.1: Room layout for empty room measurements 

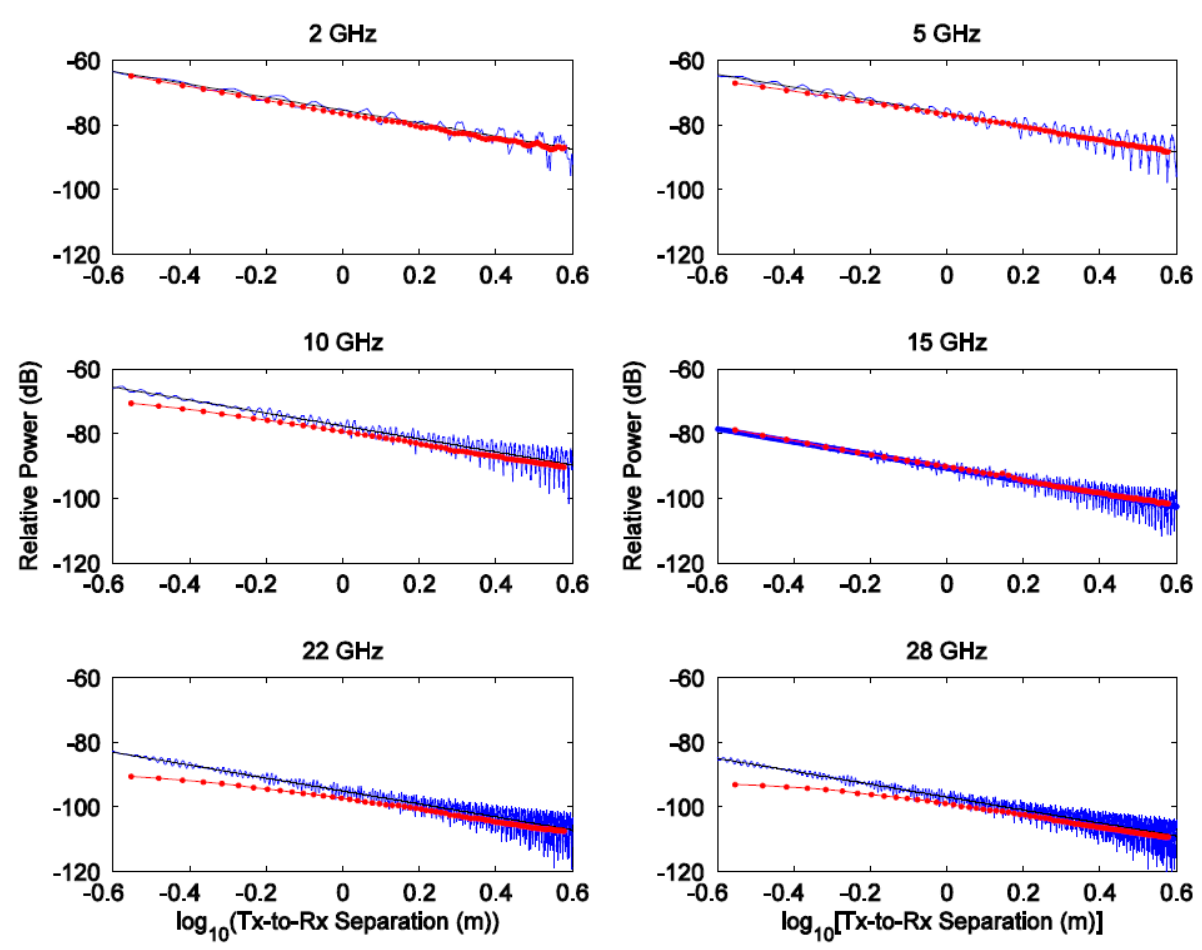

Figure 4.2: Received power as a function of $\mathrm{Tx}-\mathrm{Rx}$ separation in an empty room. Black: Friis' equation, Red: measurements, Blue: raytracing

\section{Delay Dispersion}

Measurements and modelling of RMS delay spread have been reported for indoor line-of-sight (LoS) conditions at a centre frequency of $5.2 \mathrm{GHz}$ with omnidirectional antennas [29]. The results showed that in a given room, RMS delay spread increases with distance from the transmitter up to a maximum value, and its maximum in a room is dependent on the dimensions of the room and the reflection coefficient of the walls. As proof of performance, similar experiment has been repeated in an empty room. As expected, the delay spread increases with Tx-Rx antenna separation as shown in Figure 4.3. It can also be seen that the dispersion is higher at $2 \mathrm{GHz}$. The $10 \mathrm{GHz}, 15 \mathrm{GHz}$, and $22 \mathrm{GHz}$ have almost the same mean, and the $28 \mathrm{GHz}$ has 
the lowest RMS delay spread mean. This could be due to the frequency-dependant penetration loss caused by walls, such that multipath components observed at higher frequencies came mostly from reflected waves confined to the room, while the reflected waves from neighboring rooms at $2.35 \mathrm{GHz}$ had significantly longer delays. Similar results were reported in [30], where the mean RMS delay spreads at $73 \mathrm{GHz}$ where lower than those at $28 \mathrm{GHz}$ in LoS environment.

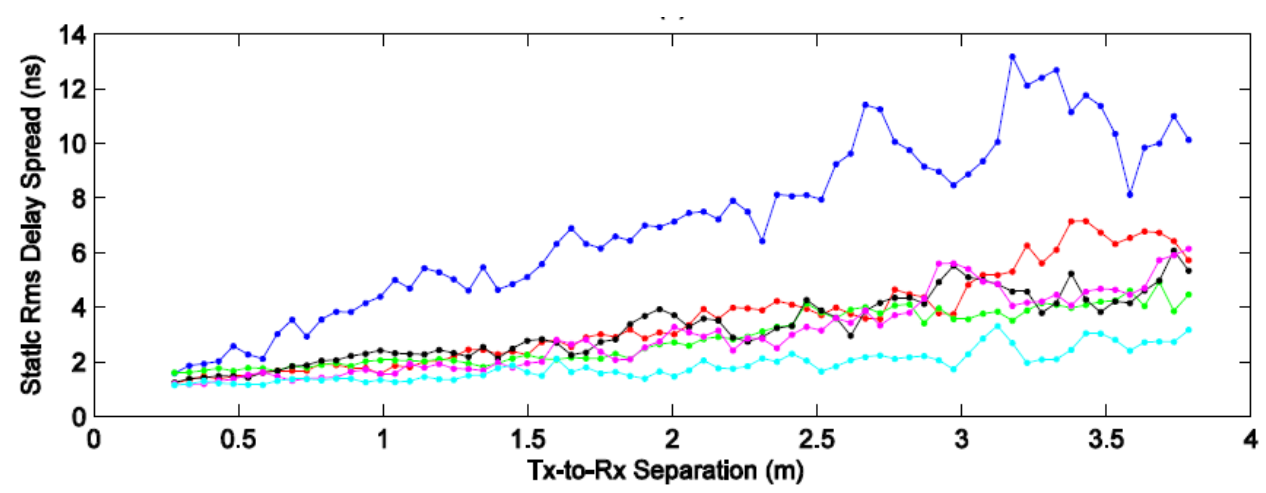

Figure 4.3: Static RMS delay spread as a function of Tx-Rx separation. Blue: $2 \mathrm{GHz}$, Red: 5GHz, Green: $10 \mathrm{GHz}$, Black: $15 \mathrm{GHz}$, Magenta: $22 \mathrm{GHz}$, Cyan: $28 \mathrm{GHz}$

\subsubsection{Diffraction Loss from One Obstruction}

Diffraction, as explained in Section 2.3, is the bending of wave-fronts around obstacles. Measuring diffraction loss is not an easy task; however, a simple model, referred to the knife-edge model reported in Section 2.2.1, can be used to predict such behaviour. Reported in this section are results from both knife-edge diffraction modelling and measurements of diffraction loss caused by a bookcase with a metallic back positioned between Tx and Rx antennas and orthogonal to a line between them. The measurement setup is shown in Figure 4.4. 


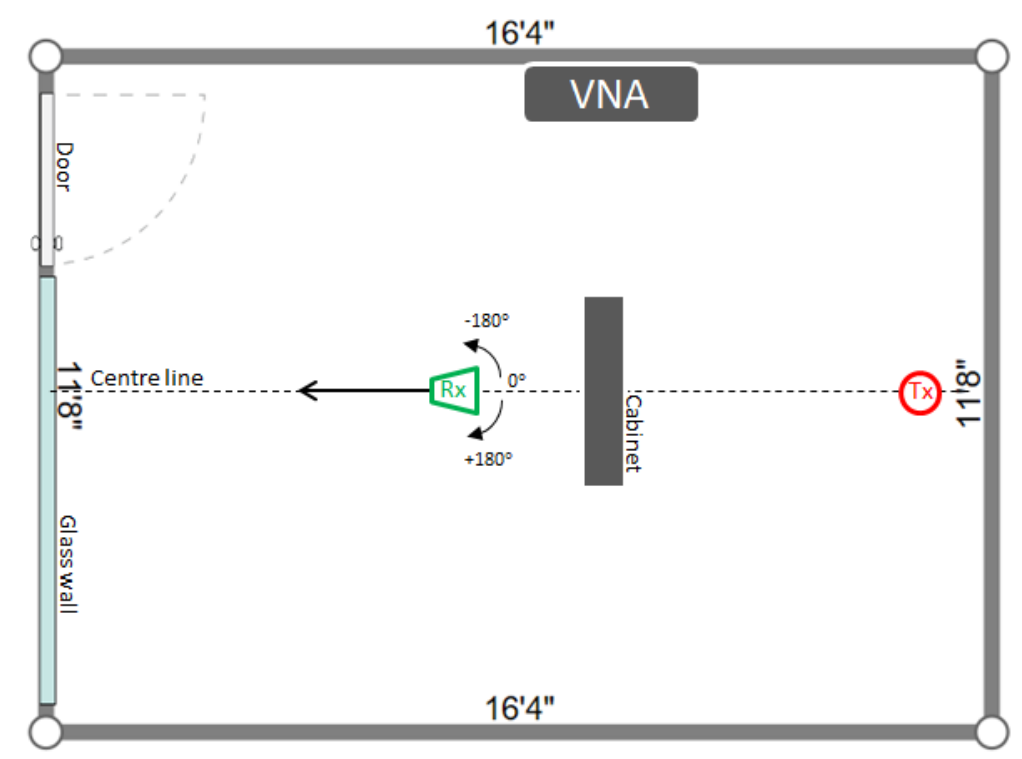

Figure 4.4: Room layout - single obstruction measurements

The transmission was from the 2-30 GHz vertically-polarized horn, positioned on the centreline of the room, $23 \mathrm{~cm}$ from the end wall. The bookcase, of depth 40.6 $\mathrm{cm}$, height $128.3 \mathrm{~cm}$, and width of $80 \mathrm{~cm}$ was positioned with its metallic back facing the Tx antenna, at $162.6 \mathrm{~cm}$ from it, and centred symmetrically on the centreline of the room. The $\mathrm{Rx}$ antenna was either the $2-18 \mathrm{GHz}$ horn or the $18-40 \mathrm{GHz}$ horn, depending on the operating frequency. The Rx antenna was mounted on a tripod at the height of $1 \mathrm{~m}$ above floor level and pointed at $0^{\circ}$ azimuth, defined as the direction along the centreline of the room towards the Tx antenna. While the Rx was being moved manually along the centreline in steps of $5 \mathrm{~cm}$ from the Tx, the VNA was used to record insertion loss across the $500 \mathrm{MHz}$ bandwidth. Figure 4.5 shows measured and modelling results for each centre frequency.

Figure 4.5 reports transmission loss between unity gain antennas $\left(L_{U G A}\right)$ in $\mathrm{dB}$ versus antenna separation in metres. The dash-dot curves show results from the 
application of Friis' equation, whereas solid curves present results from measurements in an empty room. It can be seen that the dash-dot and solid curves follow each other fairly closely. The small deviations at the beginnings of the blue and red curves (2 $\mathrm{GHz}$ and $5 \mathrm{GHz}$ ) are believed to be due to antenna pointing problems. The curves with the circle markers show results for the obstructed case. These curves are identical to the empty room results when measurements were taken between the obstruction and the Tx antenna, however, beyond the obstruction, an increase in the transmission loss with respect to the empty room results and those from Friis' equation is clear for all frequency bands.

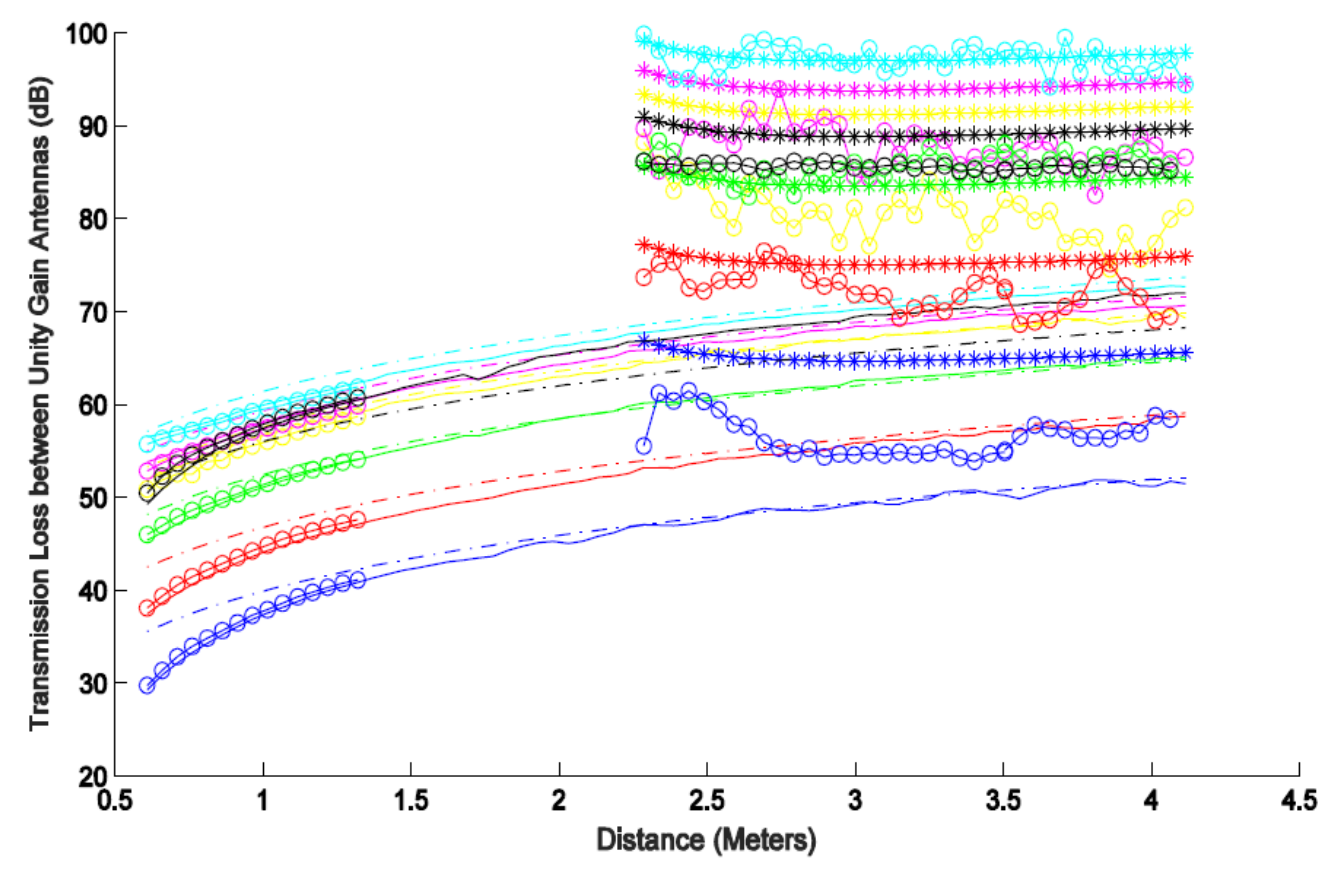

Figure 4.5: $L_{U G A}$ vs Tx-Rx antenna separation at specified centre frequencies for the case of an empty room and when a book shelf cabinet is positioned at $162.5 \mathrm{~cm}$ from an omnidirectional Tx antenna: Blue: $2.35 \mathrm{GHz}$, red 5.2 $\mathrm{GHz}$, green: $10 \mathrm{GHz}$, yellow: $18 \mathrm{GHz}$, magenta: $22 \mathrm{GHz}$, cyan: $28 \mathrm{GHz}$ 
The asterisks in Figure 4.5 are results from applying an ITU-R ${ }^{2}$ simplification of the knife-edge diffraction model, given by

$$
L_{o b s}= \begin{cases}6.9+20 \log _{10}\left(\sqrt{1+v^{2}}+v\right), & \text { if } v>0.7 \\ 0, & \text { otherwise }\end{cases}
$$

where as in Eq (2.14)

$$
v=h_{o b s}\left(\sqrt{\frac{2}{\lambda}\left(\frac{d_{T}+d_{R}}{d_{T} d_{R}}\right)}\right)
$$

where $h_{o b s}$ represents the height of the obstruction above a line connecting the Tx and Rx antennas, $d_{T}$ is the distance of the obstruction from the Tx antenna, and $d_{R}$ is the distance of the obstruction from the Rx antenna. As can be seen, the knife-edge modelling and the measured data agree exceptionally well at the frequency bands of 10 and $28 \mathrm{GHz}$. The agreement between modelling and measurement decreases for other bands, and it is worst for the $2 \mathrm{GHz}$ and $18 \mathrm{GHz}$ bands. This disagreement has not been investigated further. However, Figure 4.5 clearly shows that obstruction loss is greater for higher frequencies.

\subsubsection{Measurement of Transmission via Indirect Paths Around a Single Obstruction}

\section{Propagation Loss Characteristics}

A key point to take from the previous section is that a solution will be needed to compensate for the increasing obstruction loss at higher frequencies. One idea is to

\footnotetext{
${ }^{2}$ The International Telecommunication Union Radio Communication Sector (ITU-R)
} 
use indirect paths via reflectors and scatters to avoid obstructions. Therefore, it is essential to conduct some phenomenological measurements to check the viability of such a proposal. More precisely, there is need to check that even with the extra path loss caused by a longer indirect path around obstructions and the additional loss caused by reflection and scattering, the total transmission loss is still lower than for direct transmission through the obstruction.

The transmission loss on the indirect path around an obstruction was measured at the frequency of $10 \mathrm{GHz}$ for the case of bare walls and for different targets placed against the wall as shown in Figure 4.6.

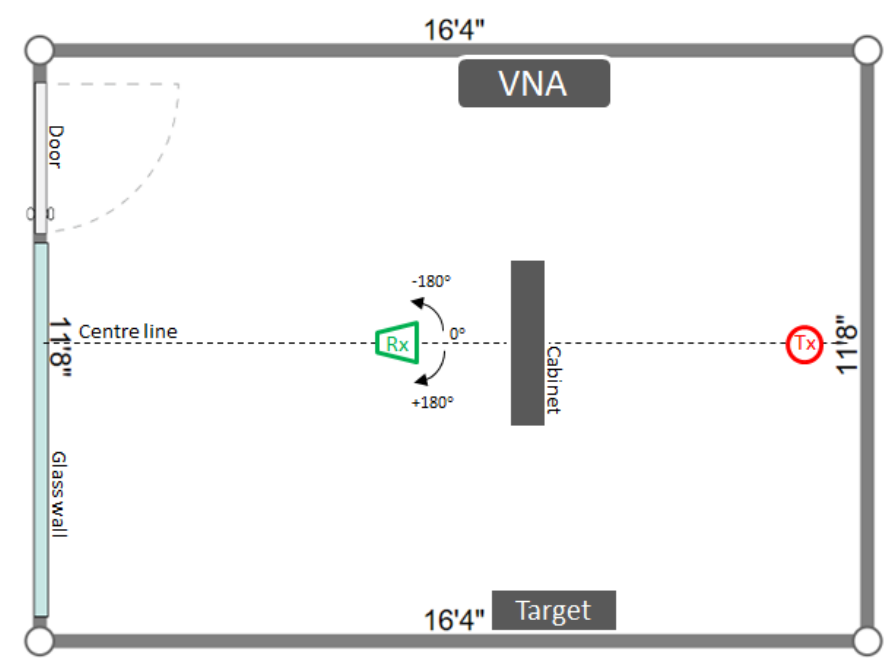

Figure 4.6: Room layout - single obstruction measurements with a reflector against the wall

Figure 4.7 shows high obstruction loss in all cases when the $\mathrm{Rx}$ horn antenna was pointed in the direction of the obstruction and the Tx antenna $\left(0^{\circ}\right)$ azimuth. A decrease in transmission loss of about $10 \mathrm{~dB}$ was observed when $\mathrm{Rx}$ antenna was pointed toward the left side wall; however, the decrease of transmission loss was 
dependent on the target against the wall when $\mathrm{Rx}$ antenna was pointed in the specular direction toward the right side wall. The decrease was the same for the cases of the bare wall and a small serving tray. For a large galvanized steel sheet the decrease was $18 \mathrm{~dB}$, and for a computer monitor, a $17 \mathrm{~dB}$ decrease was observed.

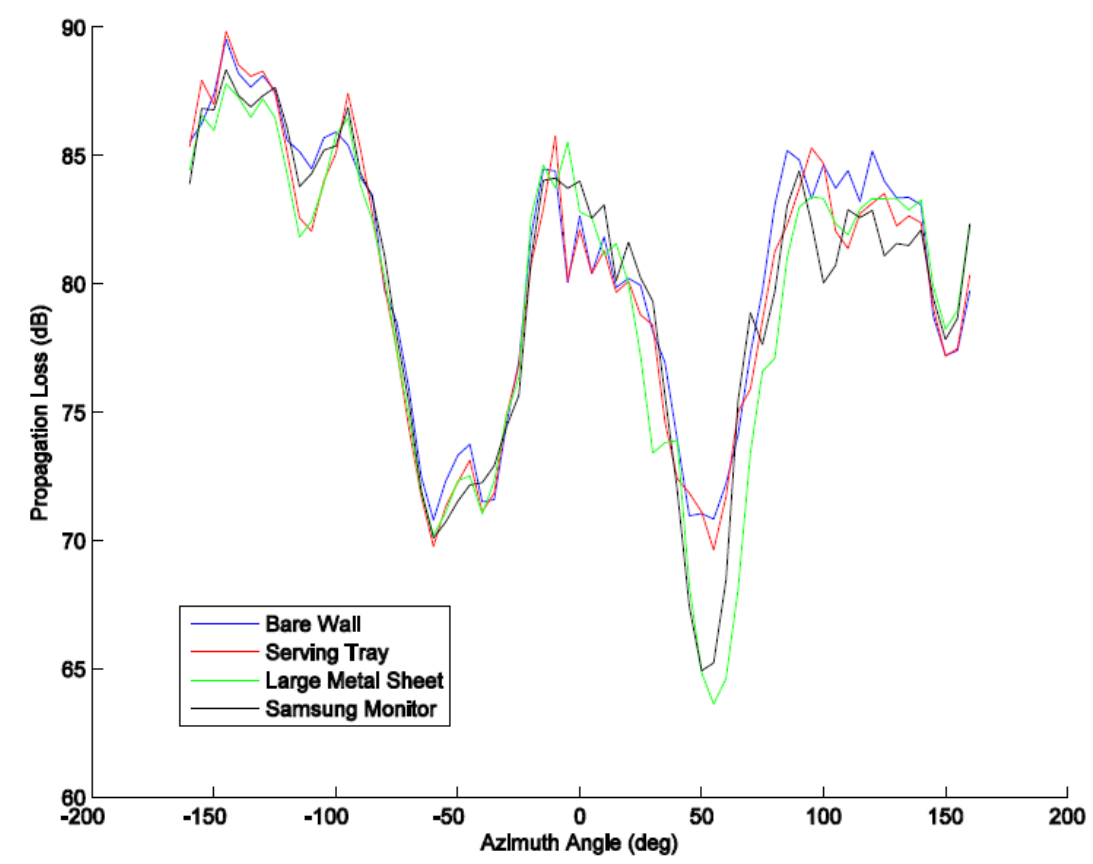

Figure 4.7: $L_{U G A}$ as a function of azimuth angle for the indirect radio path around an obstruction at $10 \mathrm{GHz}$ for the case of bare walls and for different reflectors placed against the wall.

The amount of the loss caused by reflection depends not only on the material composition and surface roughness of the reflector but also on its size in comparison with the radius of the first Fresnel zone at the location of reflector surrounding the link between the reflector and the transmitter. Since first Fresnel zone radii are smaller at higher frequencies, it is believed that there may be more good reflectors in cluttered environments that can be used to form indirect paths around obstructions at higher 
frequencies. To verify this conjecture, the same experiment was repeated, with an empty room, with bare walls and one obstruction, and with a monitor screen target and one obstruction, but for all 6 frequency bands that were studied.

The azimuth scan of averaged received powers, and corresponding transmission losses are presented in Figure 4.8 (a) and (b). In Figure 4.8 (a) a dotted red line at the VNA noise floor of $-100 \mathrm{dBw}$ is also shown. All received signals at $0^{\circ}$ azimuth were above the noise floor and therefore can be considered to be free from the influence of noise. Even though results at other azimuths were below the noise floor, it is believed that averaging over frequency after the measurements would have significantly alleviated any noise problems. The coloured $\mathrm{x}$ markers in both subplots in Figure 4.8 show results from the application of Friis' equation for free space transmission. At $0^{\circ}$ azimuth, because of the absence of furniture and averaging over frequency to mitigate multipath effects, it was expected that measurements should yield the same results as Friis' equation. By making a quick comparison, it can be seen that this is true for all frequency bands, except $15 \mathrm{GHz}$. The deviation at the $15 \mathrm{GHz}$ is believed to be due to a double-peak at boresight $\mathrm{Rx}$ antenna pattern at $15 \mathrm{GHz}$. 

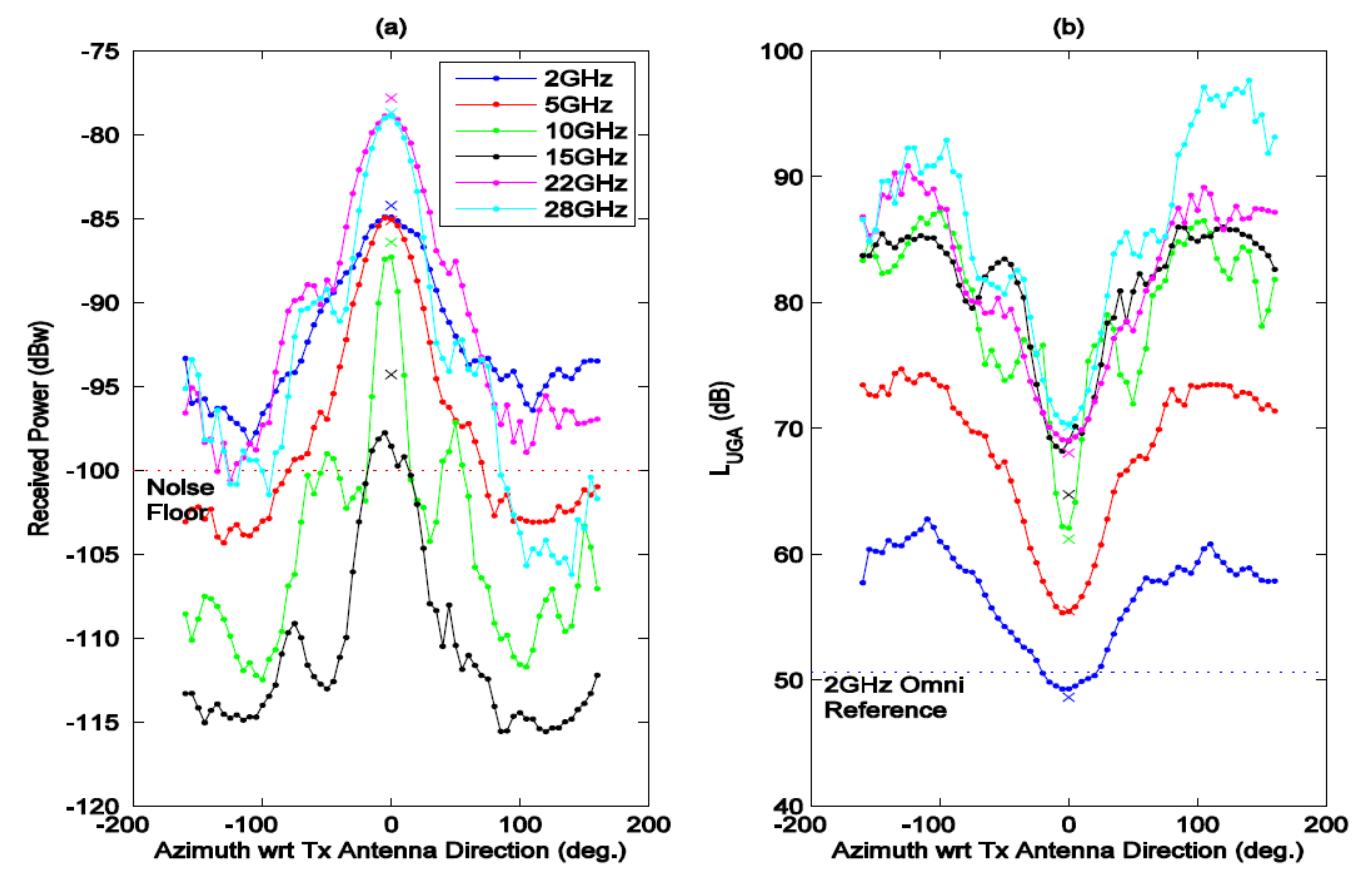

Figure 4.8: (a) Received powers and (b) $L_{U G A}$ values from an azimuth scan in the empty room

As expected, the transmission loss increases non-linearly as frequency increases. Also, shown in Figure 4.8, a measured value for received power at $2 \mathrm{GHz}$ when the $\mathrm{Rx}$ antenna was an omnidirectional quarter-wavelength monopole. This measurement can be used as a reference in comparing other results with a result which is typical of one which would be measured in current operating system.

The following results are from an experiment that was made with one obstruction. The room setup for this experiment was the same as the one described in Section 4.2 in which there was one obstruction and bare walls. In a similar format to Figure 4.8, Figure 4.9 shows the received power and transmission loss as a function of azimuth angle. It can be noted from Figure 4.9 (a) that the received power from the specular direction near $+/-45^{\circ}$ azimuth were all greater than the noise floor. Again, the 
obstruction loss increases with frequency according to Figure 4.9 (b).

An important observation from Figure 4.9 (b) is that beam-steering gain is significant only at higher frequencies, i.e., frequency bands above $6 \mathrm{GHz}$. Also interesting is the symmetry of the plot, showing reflection conditions were approximately the same from both side walls.
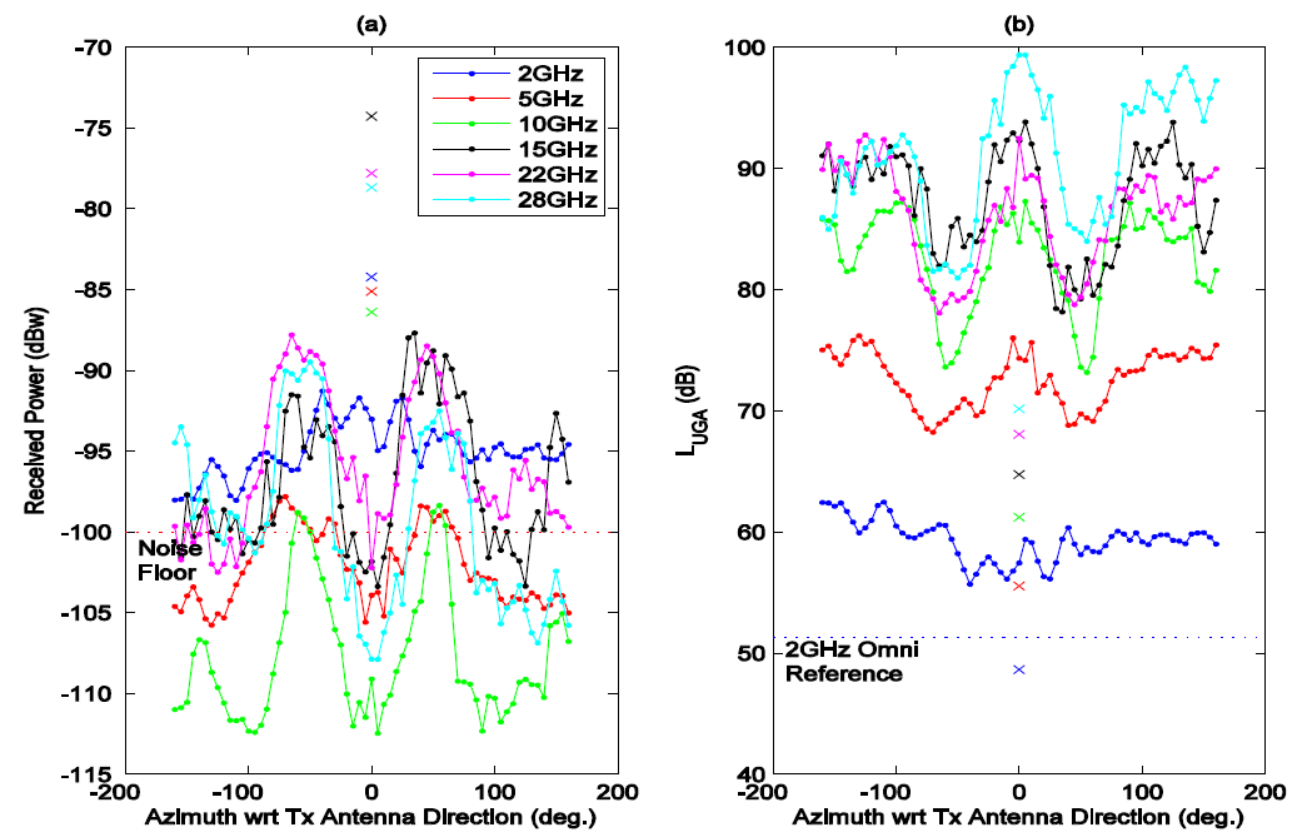

Figure 4.9: (a) Received powers and (b) $L_{U G A}$ values from an azimuth scan in the case of one obstruction and bare walls

In table $4.1 L_{o b s}$ shows the obstruction loss in the specified frequency band, calculated by subtracting the free space value from the value of $L_{U G A}$ that was measured when Rx antenna was pointed at $0^{\circ}$ azimuth. The values of $\Delta L_{1}(d B)$ are the differences in loss of the indirect path over the direct one, as it can be seen, values are all negative indicating decrease in transmission loss when Rx antenna was steering to receive a signal over indirect path around the obstruction. Noted also that the improvement 
has an increasing trend with frequency. The values of the column labelled $\Delta L_{2}(d B)$ are results of transmission loss over the strongest indirect path compared with loss incurred in the omnidirectional reference scenario at $2 \mathrm{GHz}$.

Table 4.1: Obstruction and indirect path losses in the case of a single obstruction and bare walls

\begin{tabular}{|c||c|c|c|}
\hline $\begin{array}{l}\text { Frequency } \\
{[\mathrm{GHz}]}\end{array}$ & $L_{\text {obs }}(d B)$ & $\Delta L_{1}(d B)$ & $\Delta L_{2}(d B)$ \\
\hline 2 & 9 & -3 & 7 \\
\hline 5 & 19 & -6 & 18 \\
\hline 10 & 25 & -12 & 22 \\
\hline 15 & 28 & -14 & 28 \\
\hline 22 & 18 & -14 & 27 \\
\hline 28 & 29 & -18 & 30 \\
\hline
\end{tabular}

A similar experiment was conducted, but with a monitor screen placed as a target against the wall as shown previously in Figure 4.6. As opposed to the symmetry in azimuth shown in results with bare walls before, the results presented in Figure 4.10 are asymmetrical in azimuth, showing significantly lower transmission losses in the case of a reflected signal received from the target. It can be noted that the reflected signal from the computer monitor at the 5 and $10 \mathrm{GHz}$ bands were almost same as free space results marked with (x). Gain values of all frequency bands are presented in Table 4.2 . 

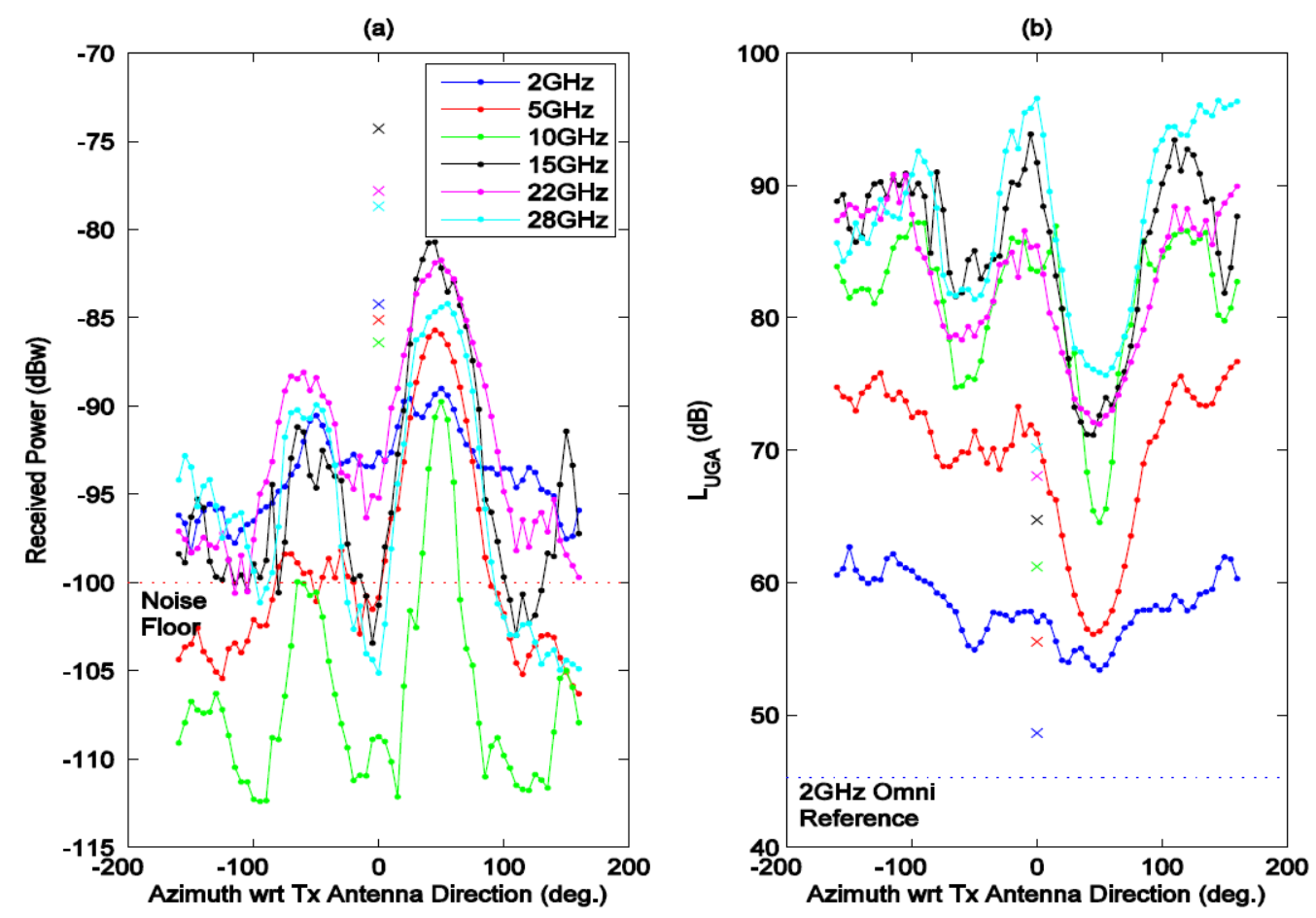

Figure 4.10: (a) Received powers and (b) $L_{U G A}$ values from an azimuth scan in the case of one obstruction and a computer monitor against the right side wall

A comparison, of the two columns that labelled $\Delta L_{1}(d B)$ in both Tables 4.1 and 4.2 , reveals that placing a monitor screen resulted in gain of $1,9,8,6,1$, and 3 decibels for the $2,5,10,15,22$, and $28 \mathrm{GHz}$ respectively. The differences in losses for the two scenarios with respect to the $2 \mathrm{GHz}$ reference case are $-1,7,2,2,0$, and 0 decibels, for the $2,5,10,15,22$, and $28 \mathrm{GHz}$ bands, respectively. These results could be due to the fact that the presence of the monitor screen also provides about $5 \mathrm{~dB}$ indirect path gain in the omnidirectional case. 
Table 4.2: Obstruction and indirect path losses in case of a single obstruction and a computer monitor placed against the right side wall

\begin{tabular}{|c||c|c|c|}
\hline $\begin{array}{l}\text { Frequency } \\
{[\mathrm{GHz}]}\end{array}$ & $L_{\text {obs }}(d B)$ & $\Delta L_{1}(d B)$ & $\Delta L_{2}(d B)$ \\
\hline 2 & 8 & -4 & 8 \\
\hline 5 & 16 & -15 & 11 \\
\hline 10 & 24 & -20 & 20 \\
\hline 15 & 29 & -20 & 26 \\
\hline 22 & 18 & -15 & 27 \\
\hline 28 & 26 & -21 & 30 \\
\hline
\end{tabular}

\section{Channel Impulse Response Characteristics}

In addition to the propagation loss characteristics, impulse response and the delay dispersion characteristics are important for the design of digital systems. As already discussed in Chapter 3, RMS delay spread is one way to quantify the impulse response delay dispersion.

Figure 4.11 shows the IREs for the case of the single obstruction with bare walls, while Figure 4.12 shows IREs for the case of the single obstruction with a computer monitor placed against the right side wall. In both figures the blue curves represent the IREs of the signal when the Rx antenna was pointed toward the azimuth from which the strongest signal was received, the red curves show the IREs in case of $0^{\circ}$ azimuth, and the black curves used as a reference in which the Rx antenna was replaced by $2 \mathrm{GHz}$ omnidirectional antenna. It can be seen from both figures that 
multipath signal energy is greater at lower frequencies, i.e. at $2 \mathrm{GHz}$, and also greater for the case of $2 \mathrm{GHz}$ omni reference (dashed-black). In addition, by comparing the first multipath components in both figures, it can be seen that the peak is higher in the case of monitor screen placed against the wall than the case of bare walls. Furthermore, as the frequency increases, i.e. from $2 \mathrm{GHz}$ to $28 \mathrm{GHz}$, the difference between the peaks of the red curve $A z=0^{\circ}$ and the blue curve $A z=A z_{\text {max }}$ increases, which agrees with the obstruction loss results reported earlier.
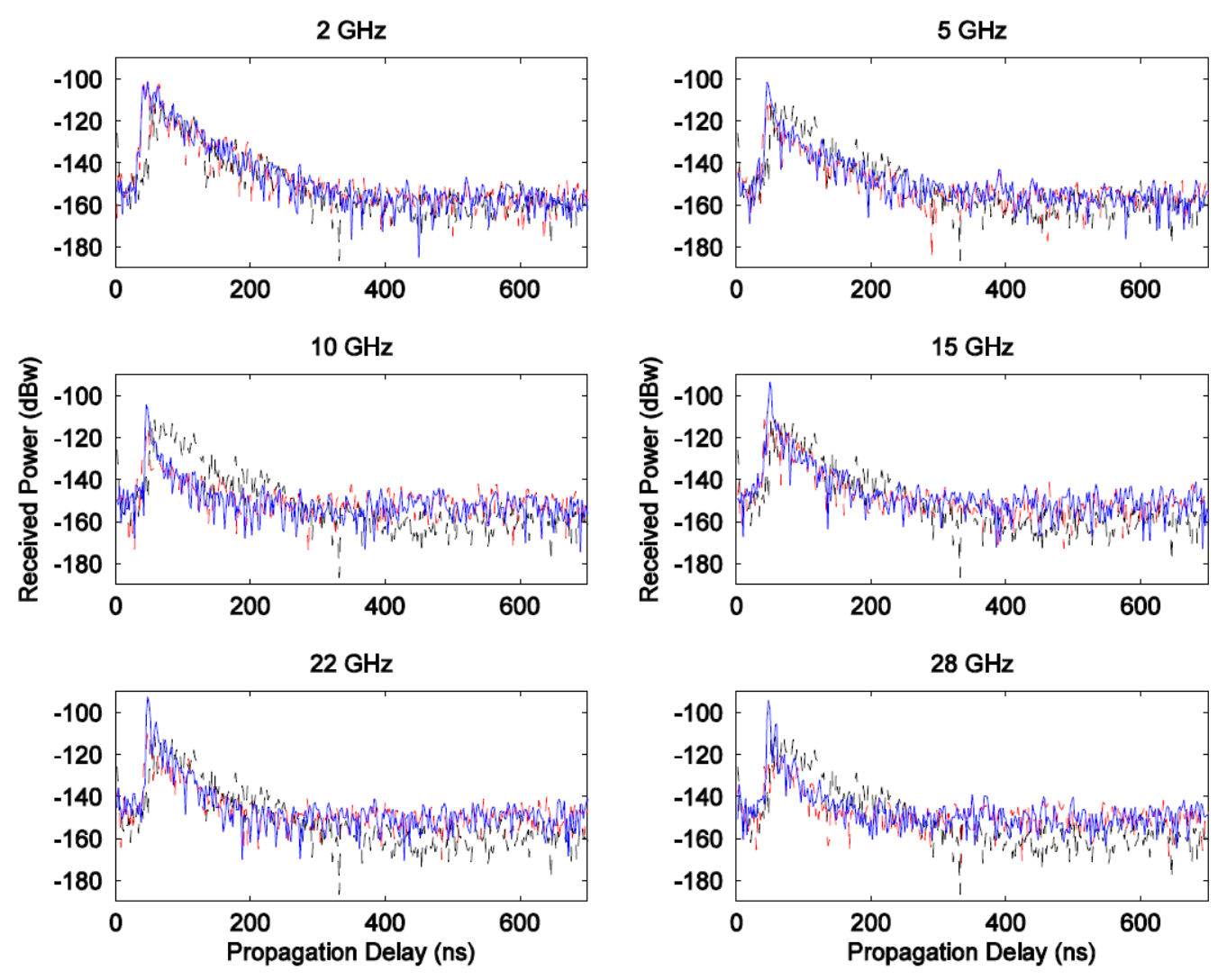

Figure 4.11: IREs for the case of one obstruction and bare walls, Blue: $A z=A z_{\text {max }}$, Red: $A z=0^{\circ}$, Black: $2 \mathrm{GHz}$ omni reference 

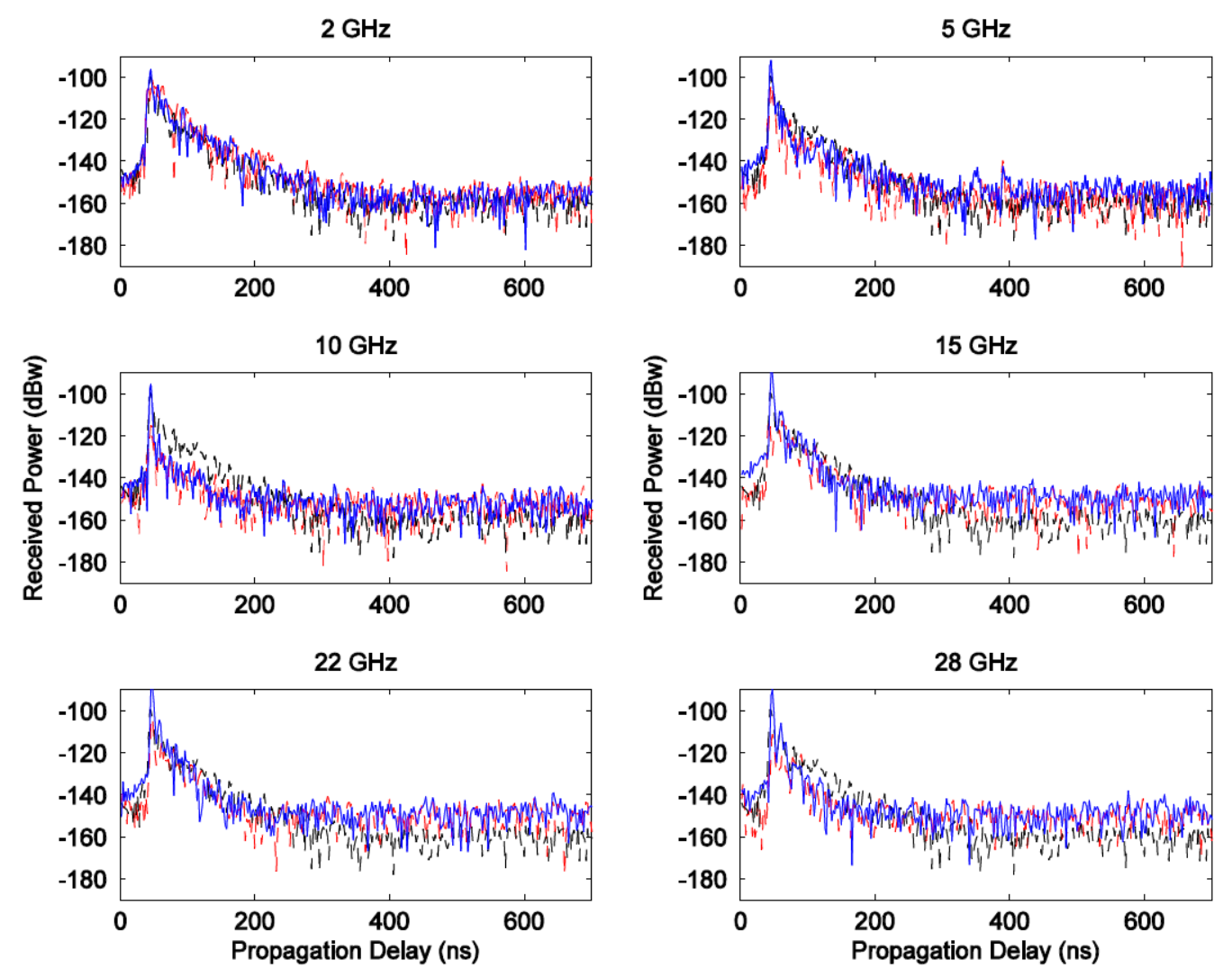

Figure 4.12: IREs for the case of one obstruction and a reflector placed against the wall, Blue: $A z=A z_{\max }$, Red: $A z=0^{\circ}$, Black: $2 \mathrm{GHz}$ omni reference

Table 4.3 lists all RMS delay spread values for cases of an empty room, one obstruction with bare walls, and one obstruction with computer monitor against the wall. Four main observations can be made from Table 4.3. First, in the case of an empty room, the maximum power is always received from the LoS which is in the direction of $0^{\circ}$ azimuth, the RMS delay spreads for $A z=0^{\circ}$ and $A z=A z_{\max }$ are the same as a result. Second, the RMS delay spread values decrease with frequency in the range from $2 \mathrm{GHz}$ to $15 \mathrm{GHz}$. There is a jump at $22 \mathrm{GHz}$ and then another decrease at $28 \mathrm{GHz}$ with respect to the value at $22 \mathrm{GHz}$. The jump at $22 \mathrm{GHz}$ is believed to be because the beamwidth of the horn antenna used at the upper two 
frequencies was greater than that of the horn used at the lower frequencies. Third, RMS delay spreads are considerably higher at $A z=0^{\circ}$ than at $A z=A z_{\max }$ in the obstructed case. Fourth, it can be seen that when $A z=A z_{\max }$ in the case of obstruction with the computer monitor as a target RMS delay spread are almost the same for all frequencies except at $2 \mathrm{GHz}$. It thus appears that when there is a good reflector which causes a strong indirect path link RMS delay spreads become frequency-independent. 
Table 4.3: RMS delay spreads

\begin{tabular}{|c|c|c|}
\hline $\begin{array}{l}\text { Frequency } \\
{[\mathrm{GHz}]}\end{array}$ & $\sigma_{\tau s}(n s)\left(A z=0^{\circ}\right)$ & $\sigma_{\tau s}(\mathrm{~ns})(\mathrm{Az}=\mathrm{Az}-\mathrm{max})$ \\
\hline \multicolumn{3}{|c|}{ Empty room (LoS) } \\
\hline 2 & 9 & 9 \\
\hline 5 & 5 & 5 \\
\hline 10 & 4 & 4 \\
\hline 15 & 3 & 3 \\
\hline 22 & 4 & 4 \\
\hline 28 & 2 & 2 \\
\hline \multicolumn{3}{|c|}{ One obstruction with bare walls } \\
\hline 2 & 16 & 15 \\
\hline 5 & 17 & 9 \\
\hline 10 & 20 & 5 \\
\hline 15 & 18 & 4 \\
\hline 22 & 20 & 6 \\
\hline 28 & 13 & 4 \\
\hline \multicolumn{3}{|c|}{ One obstruction with computer monitor } \\
\hline 2 & 15 & 11 \\
\hline 5 & 9 & 3 \\
\hline 10 & 25 & 2 \\
\hline 15 & 18 & 3 \\
\hline 22 & 11 & 3 \\
\hline 28 & 16 & 3 \\
\hline
\end{tabular}




\subsubsection{Measurement of Transmission via Opening Between Two Obstruc- tions}

\section{Propagation Loss Characteristics}

Another phenomenon that considered to be an enabling factor to improve coverage above $6 \mathrm{GHz}$ in cluttered environments is transmitting through openings between objects. Again this can be explained theoretically by the fact that first Fresnel radii are smaller at higher frequencies; therefore, obstruction losses caused by transmission through openings between obstructions should be lower. An experiment that was designed to demonstrate this phenomenon was conducted as follows: two bookcases, similar to the one used in the previous section, were placed across the middle of the small room with $15 \mathrm{~cm}$ gap between them. The Tx and $\mathrm{Rx}$ were placed at the same location as reported in Section 4.3.1. As shown in Figure 4.13, with Rx antenna pointed at $0^{\circ}$ azimuth, the antenna was also pointed toward the opening between the two bookcases. Azimuth scans were conducted with Rx antenna as in the foregoing sections.

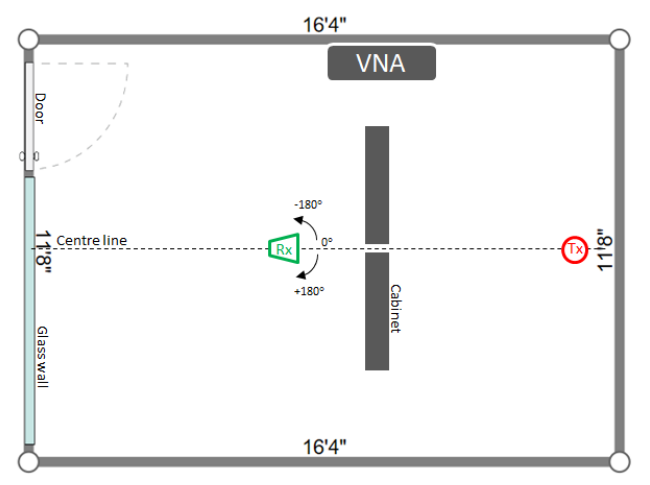

Figure 4.13: Room layout - two-obstruction measurements 
Frequency-averaged received power and transmission loss as a function of Rx antenna pointing direction in azimuth is presented in Figure 4.14. As expected, transmission loss decreases with increasing frequency. In fact, the $15 \mathrm{~cm}$ gap was explicitly chosen to provide first Fresnel clearance for frequencies at $5 \mathrm{GHz}$ and above, but not at $2 \mathrm{GHz}$ so that a valid comparison can be made. The radius of the $n_{t h}$ Fresnel zone is given by

$$
R_{n}=\sqrt{\frac{n \lambda d_{T} d_{R}}{d_{T}+d_{R}}}
$$

where $\lambda$ is the wavelength, $d_{T}$ and $d_{R}$ are the distance from the transmitter and the receiver, respectively. It can be seen from the equation that for higher frequencies with smaller wavelengths, the $n_{t h}$ Fresnel zone radius decreases.

It is clear that there was a loss with regard to the free space at the $2 \mathrm{GHz}$ even with LoS. This is due to the opening that was less than twice the radius of the first Fresnel zone. However, as wavelengths decreased, losses also decreased and were very close to free space at $15 \mathrm{GHz}$. Some gain was observed at $22 \mathrm{GHz}$ and $28 \mathrm{GHz}$ which is believed to be caused by constructive phase relationship from echos that reflected from the edge of the bookcase. 

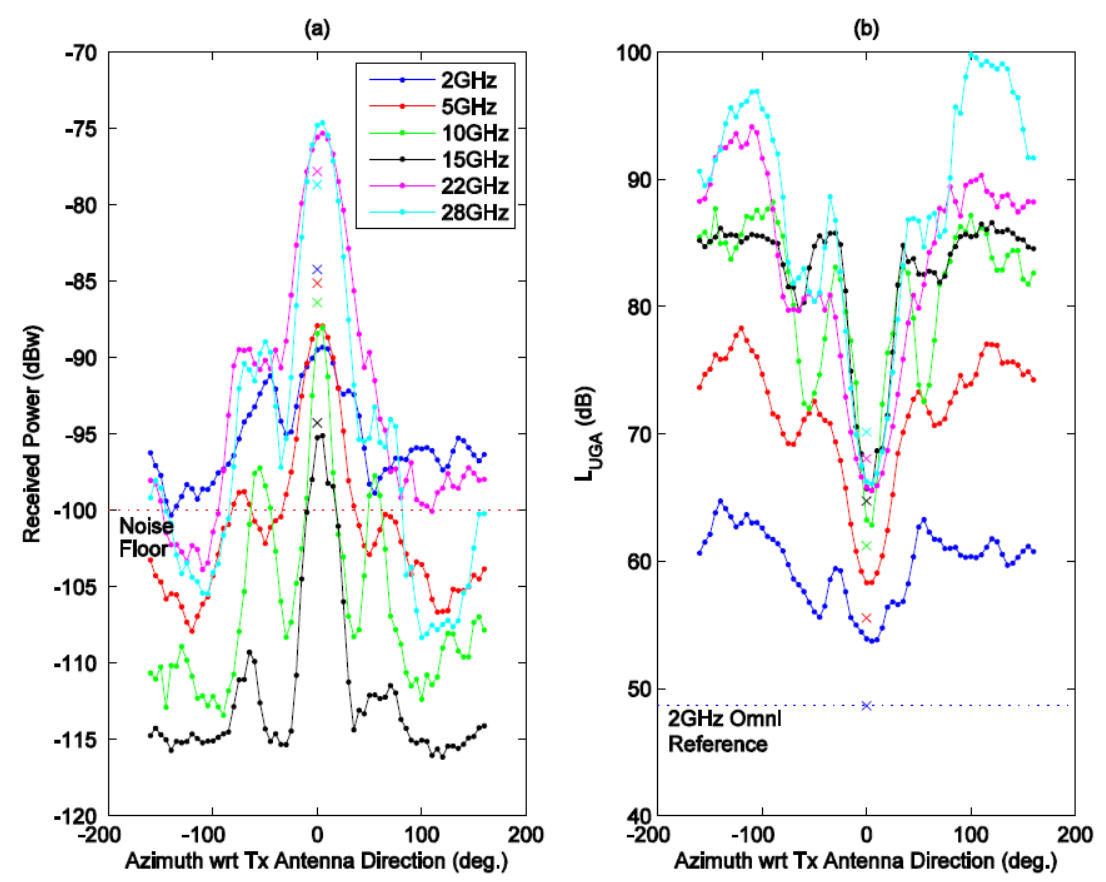

Figure 4.14: (a) Received powers and (b) $L_{U G A}$ values from an azimuth scan in the case of two-obstruction measurements

Table 4.4 summarizes the transmission loss and the $2_{n d}$ Fresnel zone radius in all the frequency bands under study. It can be seen that measured loss column at $0^{\circ}$ and the $2_{n d}$ Fresnel zone radius are proportional. 
Table 4.4: Transmission loss incurred by propagation through an opening between two bookcases

\begin{tabular}{|c||c|c|}
\hline $\begin{array}{l}\text { Frequency } \\
{[\mathrm{GHz}]}\end{array}$ & Measured Loss $(\mathrm{dB})$ & 2 x Fresnel zone Radius $(\mathrm{cm})$ \\
\hline 2 & 5.4 & 52.44 \\
\hline 5 & 2.8 & 35.25 \\
\hline 10 & 1.4 & 25.42 \\
\hline 15 & 0.9 & 20.76 \\
\hline 22 & -2.5 & 17.14 \\
\hline 28 & -4.2 & 15.19 \\
\hline
\end{tabular}

\section{Channel Impulse Response Characteristics}

Impulse response estimates were measured for all frequency bands for the case of $15 \mathrm{~cm}$ opening between two bookcases while the RX horn antenna was pointed in the direction of the transmitter. It can be seen in Figure 4.15 that maximum power was received from $0^{\circ}$ azimuth. It can also be noted that the energy at the earliest delay in the $2 \mathrm{GHz}$ reference case is lower than that at the same frequency when the horn antenna received the signal through the aperture because of the higher gain of the horn antenna. Also, the $2 \mathrm{GHz}$ reference case shows more extended multipath reception compared with other cases. 

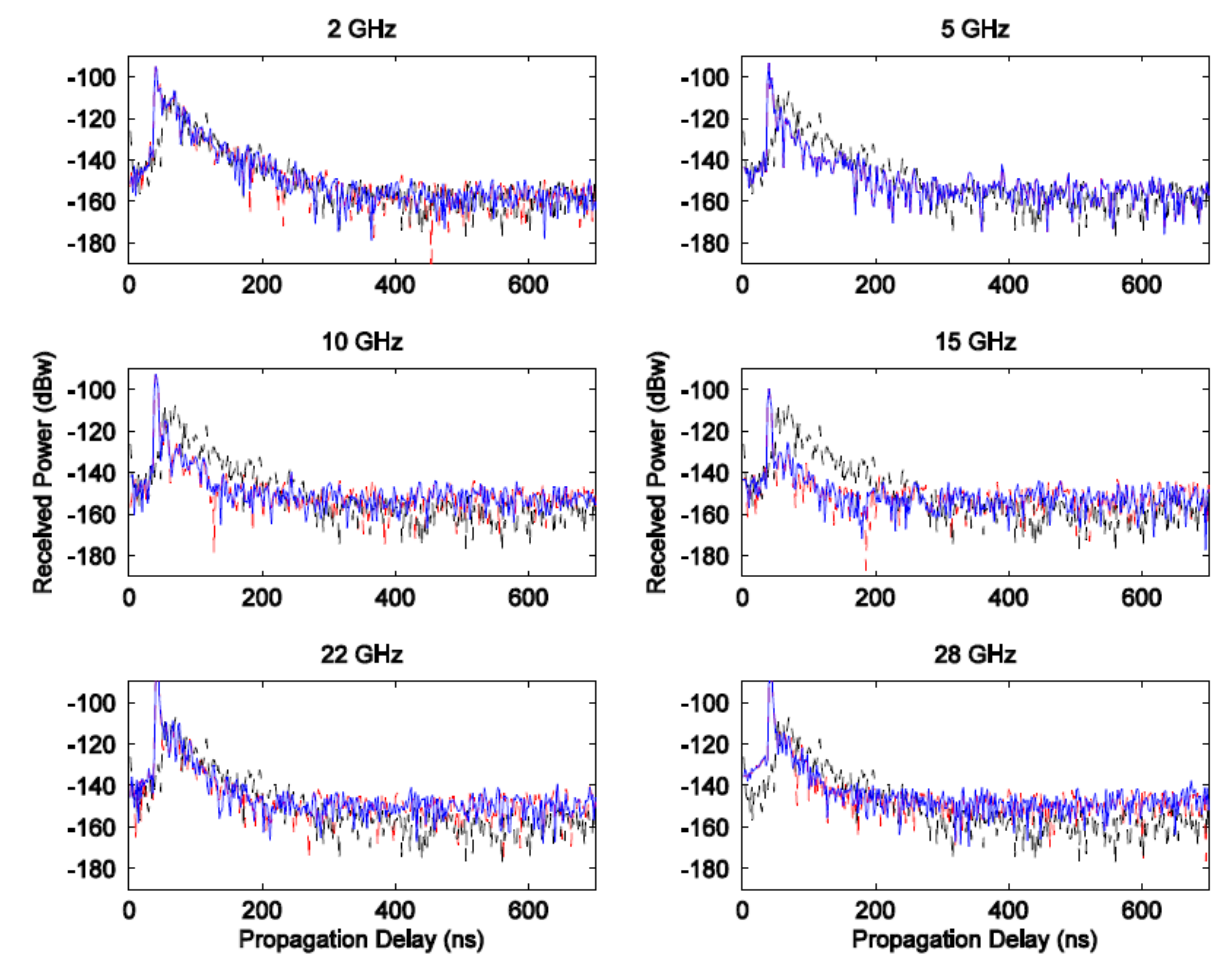

Figure 4.15: IREs for the case of a $15 \mathrm{~cm}$ opening between two book cases placed symmetrically about LoS between the Tx and Rx, Blue: $A z=A z_{\max }$, Red: $A z=0^{\circ}$, Black: $2 \mathrm{GHz}$ omni reference

Delay dispersion values are summarized in Table 4.5. It can be noted from the table that RMS delay spreads decreased as frequency increased. This also can be related to the opening width between the two bookcases and the first Fresnel zone radius allowing an increase in the power received over the direct path at higher frequencies when Fresnel zone radius becomes comparable to the opening size. 


\subsection{PROPAGATION MEASUREMENTS IN CLUTTER AND}

CHANNEL MODELLING

Table 4.5: Delay dispersion values for the case of a $15 \mathrm{~cm}$ aperture between two bookcases

\begin{tabular}{|c||c|}
\hline $\begin{array}{l}\text { Frequency } \\
{[\mathrm{GHz}]}\end{array}$ & $\begin{array}{l}\text { Static RMS de- } \\
\text { lay spread (ns) }\end{array}$ \\
\hline 2 & 10 \\
\hline 5 & 4 \\
\hline 10 & 2 \\
\hline 15 & 2 \\
\hline 22 & 2 \\
\hline 28 & 1 \\
\hline
\end{tabular}

\subsection{Propagation Measurements in Clutter and Channel Modelling}

This section discusses measurements that were made in the same room where the phenomenological measurements were made, however, furniture was added, as shown in Figure 4.16 and 4.17, to emulate a cluttered small office environment. The furniture in the room included 6 study desk workstations with computer monitors on them and office chairs in front of them, pictures on the wall, an emulated office partition made with tin-foil-covered plywood, and a large TV monitor. 


\subsection{PROPAGATION MEASUREMENTS IN CLUTTER AND}

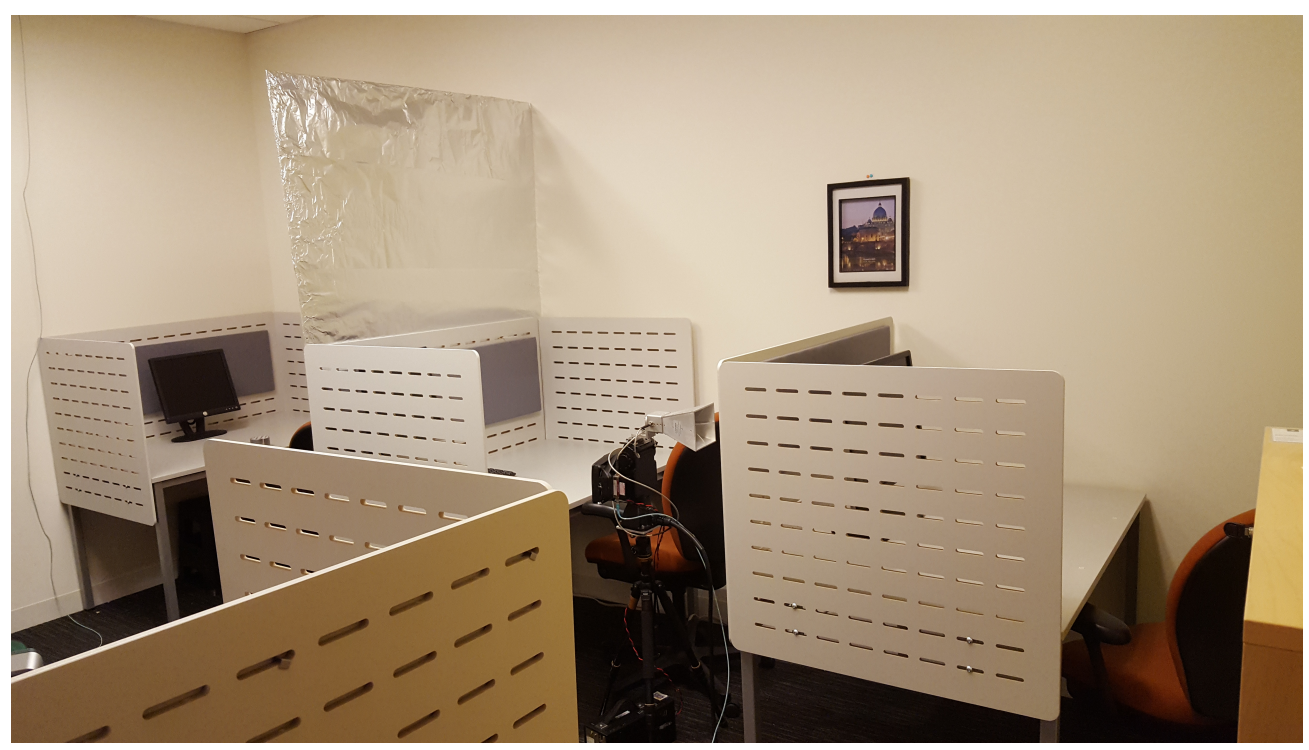

Figure 4.16: Photograph showing the furniture in the room and the 2-18 GHz horn mounted on the pan-tilt unit

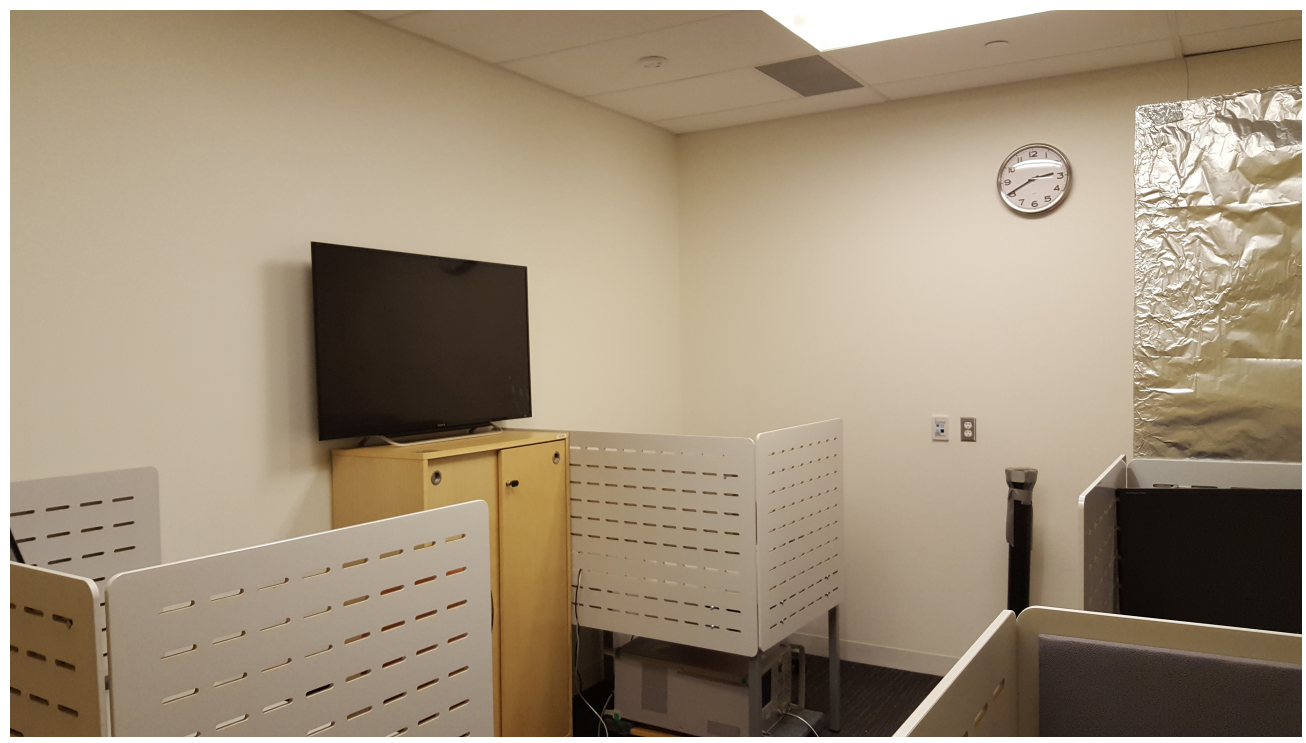

Figure 4.17: Photograph showing the furniture in the room and the biconical antenna

Measurements were conducted in which the VNA was stepped across $500 \mathrm{MHz}$ to obtain data in two different scenarios: 


\subsection{PROPAGATION MEASUREMENTS IN CLUTTER AND CHANNEL MODELLING}

\section{Scenario 1: Omni-Omni desktop to desktop measurements at $2 \mathrm{GHz}$}

In this scenario a vertically polarized $2-30 \mathrm{GHz}$ biconical antenna at desktop height 90-cm was used, the Tx antenna had omnidirectional $\mathrm{H}$ plane pattern and doublelobed E plane pattern. The reception was at 45 desktop height locations scattered throughout the room using an omnidirectional quarter-wavelength monopole antenna. The Rx antenna was either positioned on a desktop by taping it at the end of a plastic pipe $25 \mathrm{~cm}$ high or extended upward from the floor in other locations, by taping it at the end of 90-cm high plastic pipe mounted on a Christmas tree stand. The desktop and tree-stand Rx antenna locations are depicted in Figure 4.18.

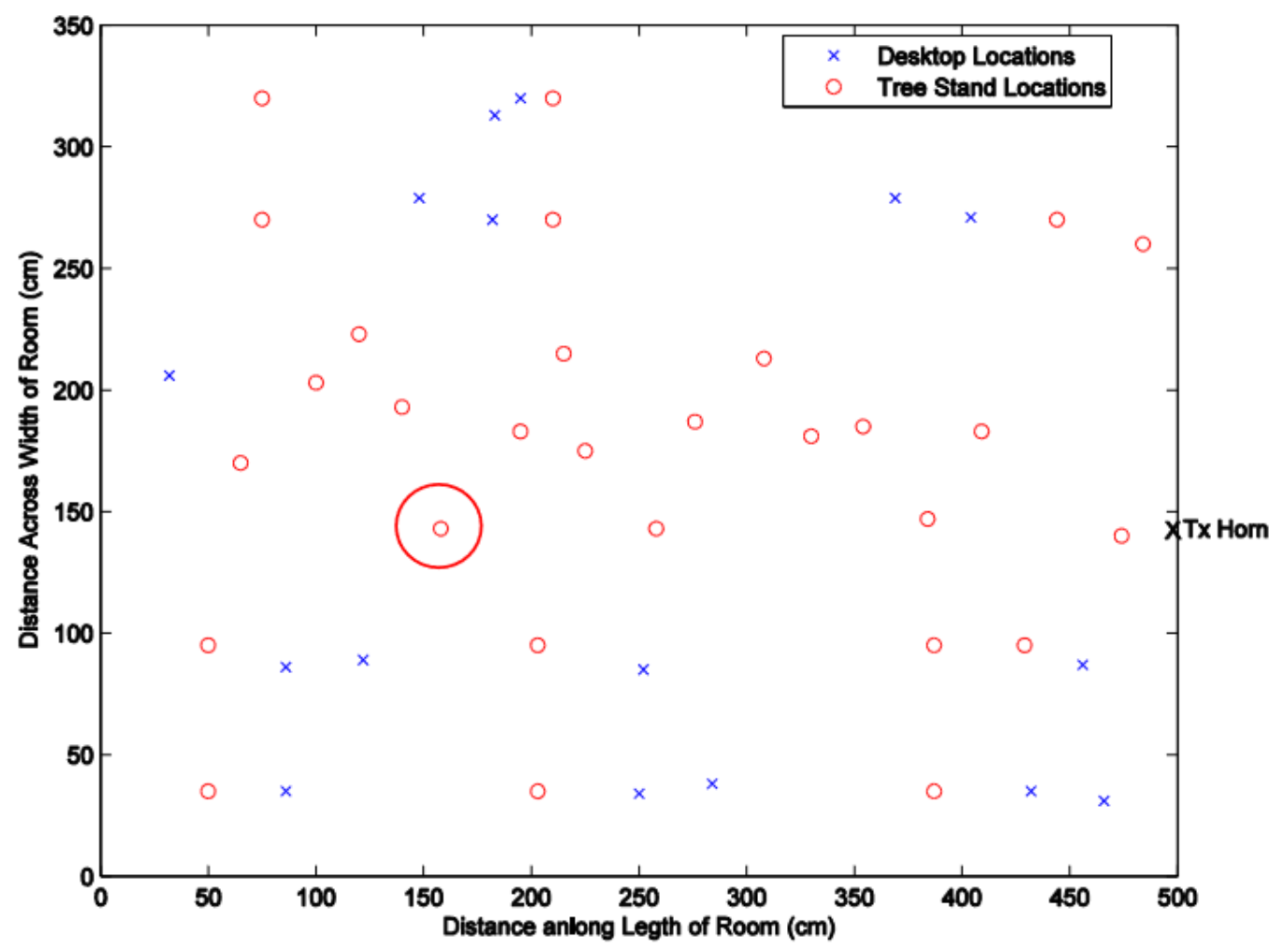

Figure 4.18: Coverage measurement locations in the cluttered room 


\subsection{PROPAGATION MEASUREMENTS IN CLUTTER AND}

\section{Scenario 2: Horn-Horn ceiling to desktop measurements at $18 \mathrm{GHz}$ and $28 \mathrm{GHz}$}

In this scenario transmission was by an $18-40 \mathrm{GHz}$ horn antenna placed above the suspended ceiling as shown in Figure 4.19. Reception at $18 \mathrm{GHz}$ and $28 \mathrm{GHz}$ was at the same 45 desktop height locations of Scenario 1. At each location, the Rx antenna scanned in azimuth and elevation from -180 to +170 degrees and from -50 to +60 degrees, respectively, in steps of 10 degrees.

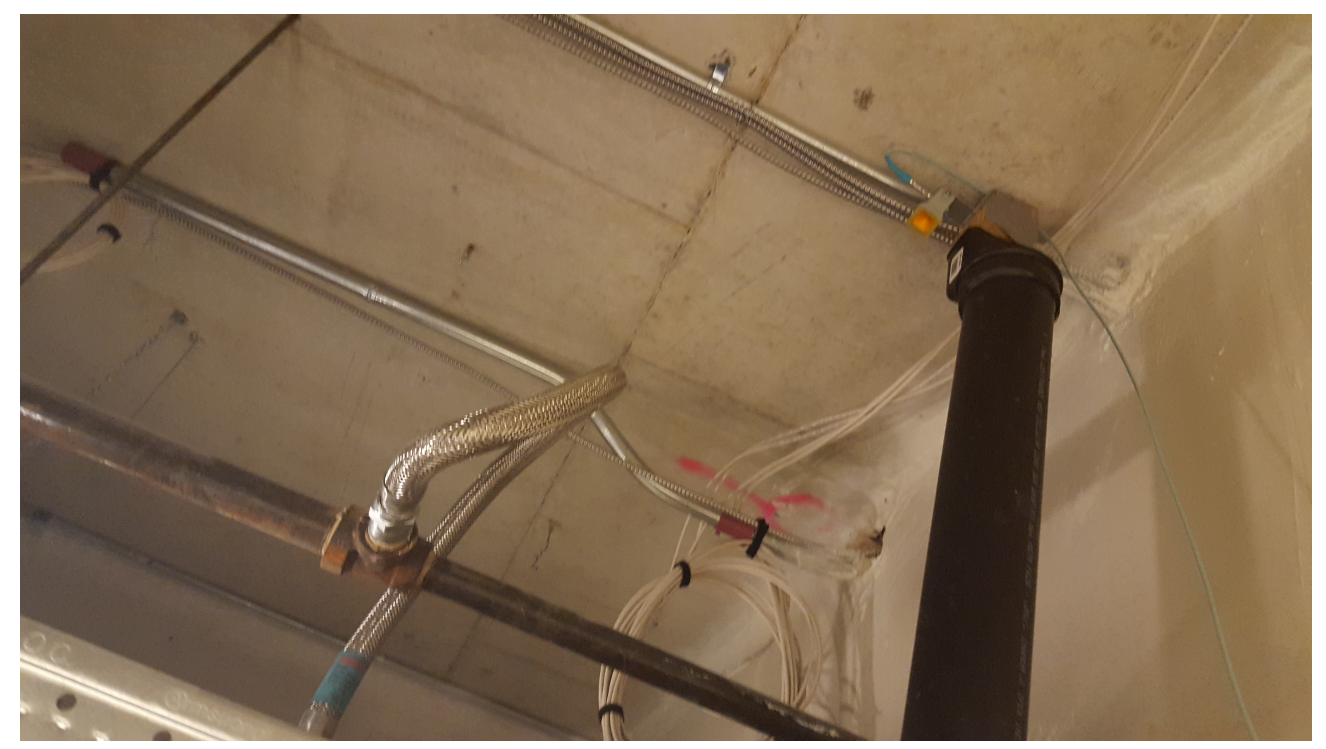

Figure 4.19: Photograph showing the mounted location of the Tx antenna

\subsubsection{Transmission Loss and Shadowing}

For the analysis of transmission loss, insertion loss measurements from the calibrated VNA system were adjusted for antenna gains, and analyzed as $L_{U G A}$, values, so they could later be used with any antenna gains of interest in systems calculations. Antenna gains, and pointing angles were all considered in these calculations. Figure 


\subsection{PROPAGATION MEASUREMENTS IN CLUTTER AND}

CHANNEL MODELLING

4.20 shows $L_{U G A}$ values calculated from the measurements and free space path loss in the $2 \mathrm{GHz}$ band for the case Scenario 1 . It should be noted that only at 5 locations the measurements have the same values as free space. Excess losses beyond free-space transmission loss ranged as high as $20 \mathrm{~dB}$ as can be seen from the green curve in the figure.

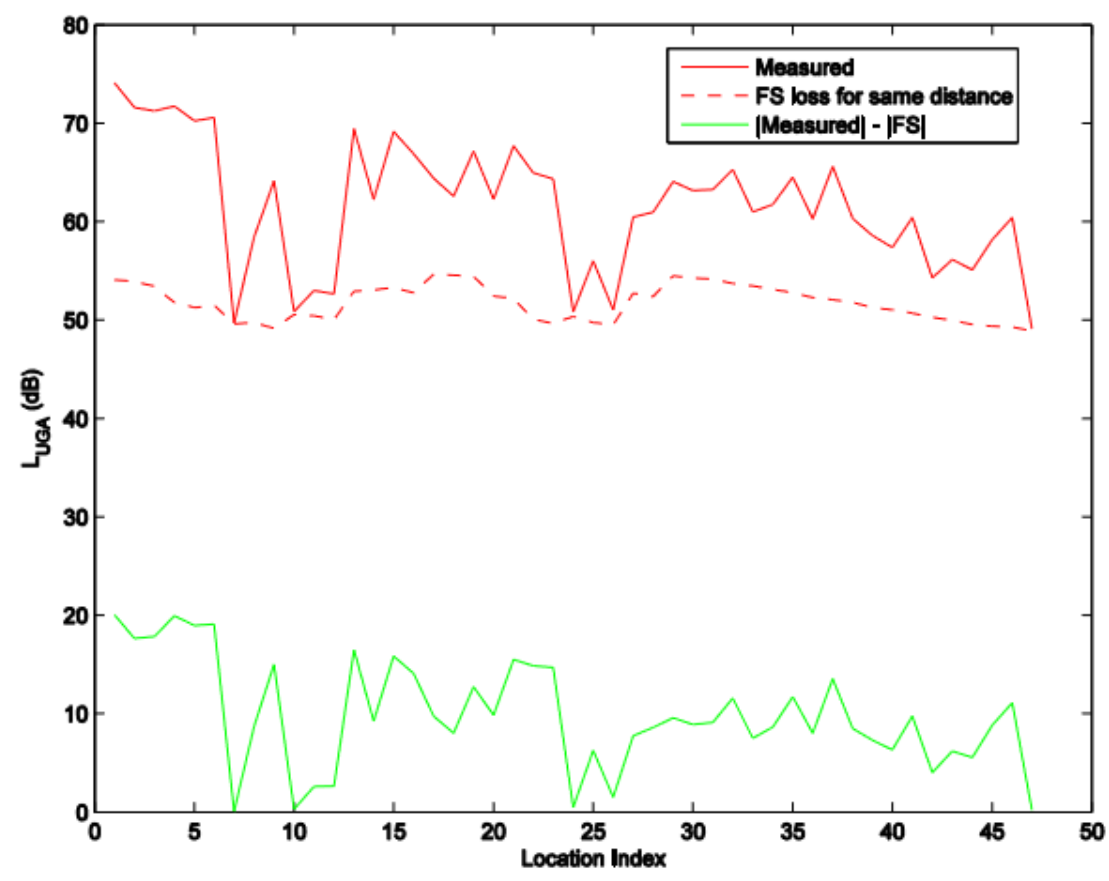

Figure 4.20: Values of $L_{U G A}$ and free space loss in the $2 \mathrm{GHz}$ band as per Scenario 1 


\subsection{PROPAGATION MEASUREMENTS IN CLUTTER AND}

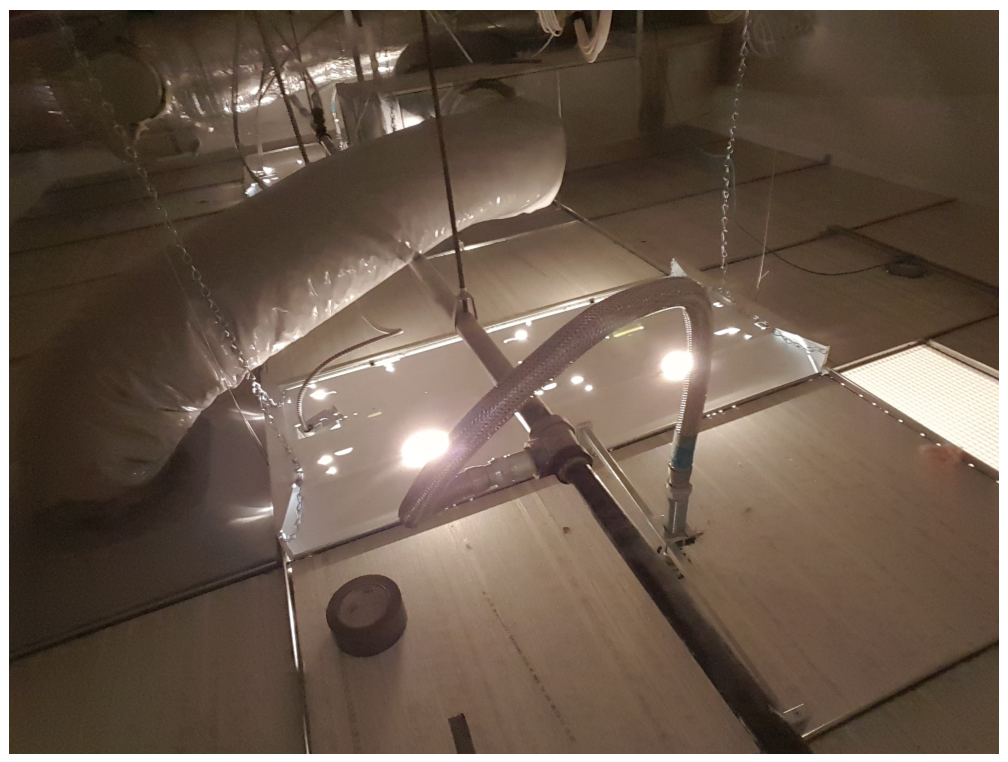

Figure 4.21: Photograph showing the view from the Tx horn antenna location above the suspended ceiling

In the coverage Scenario 2, the 18-40 GHz horn was used as the Tx antenna to emulate a sector coverage antenna at $18 \mathrm{GHz}$ and $28 \mathrm{GHz}$ above the ceiling tiles. Reception was at desk height, with the $2-18 \mathrm{GHz}$ horn for the $18 \mathrm{GHz}$ band and 18-40 GHz horn for the $28 \mathrm{GHz}$ band measurements. Both antennas scanned the azimuth and elevation, requiring 3.5 hours at each of the $45 \mathrm{Rx}$ locations at $18 \mathrm{GHz}$ band and the 22 locations at $28 \mathrm{GHz}$ band. Figure 4.22 shows the $L_{U G A}$ vs pointing direction in azimuth and elevation for location F18 circled on Figure 4.18. 


\subsection{PROPAGATION MEASUREMENTS IN CLUTTER AND}

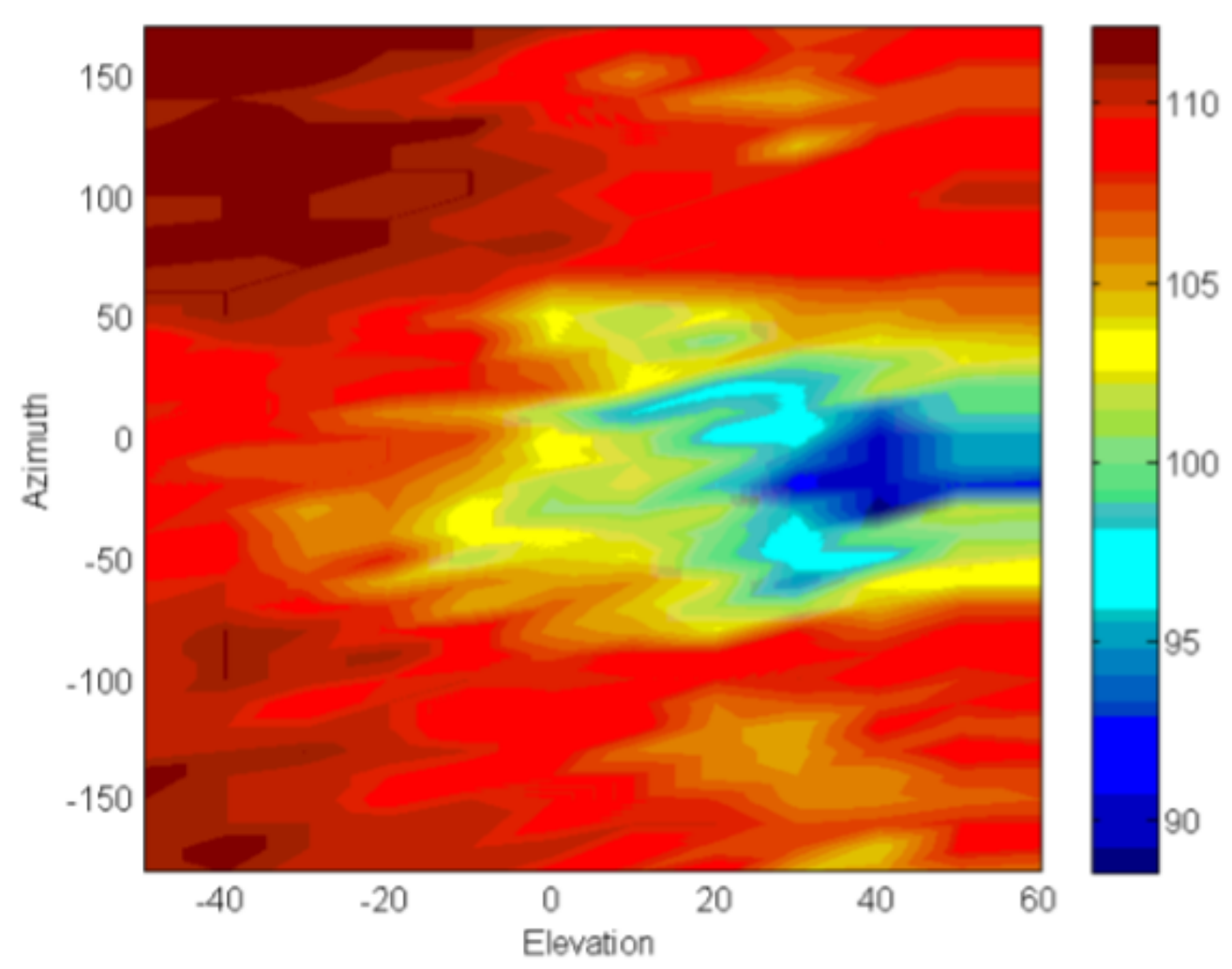

Figure 4.22: Colour-coded values of $L_{U G A}$ as a function of $\mathrm{Rx}$ antenna pointing direction at $18 \mathrm{GHz}$ with illumination by the emulated sector antenna above the ceiling tiles

As can be seen, the lowest transmission loss was at an azimuth angle of around $0^{\circ}$ and an elevation angle of almost $40^{\circ}$. This is the direction to the Tx antenna from this location as shown in Figure 4.18. It is important to note that the Rx antenna beamwidth is $10^{\circ}$ which causes the system to have $10^{\circ}$ angular resolution. Therefore, the objective of this measurements was to characterize the propagation channel for the case of a user device at each Rx location with a steerable antenna having $10^{\circ}$ azimuth and elevation beamwidth, and the channel characteristics for the direction of minimum $L_{U G A}$ were analyzed. 


\subsection{PROPAGATION MEASUREMENTS IN CLUTTER AND CHANNEL MODELLING}

For each of the measurement scenarios, frequency averaged values for the direct received power at $2 \mathrm{GHz}$, and for the direction of maximum received power at 18 and $28 \mathrm{GHz}$ were plotted in Figures 4.23-4.25 as a function of the logarithm of direct-path Tx-Rx antenna separation, and fit to a linear model using standard LMS regression analyses. Also shown in Figures 4.24 and 4.25 results from direct link measurements where the UE antenna beams were always pointed toward the Tx antenna. In this case $L_{U G A}$ values were found to be considerably greater, with the transmission loss reference values being $98 \mathrm{~dB}$ and $116 \mathrm{~dB}$ at $18 \mathrm{GHz}$, and $28 \mathrm{GHz}$, respectively, and the corresponding transmission loss exponents being 4.7 and 2.7. Results from Friis' equation are also shown in these figures.

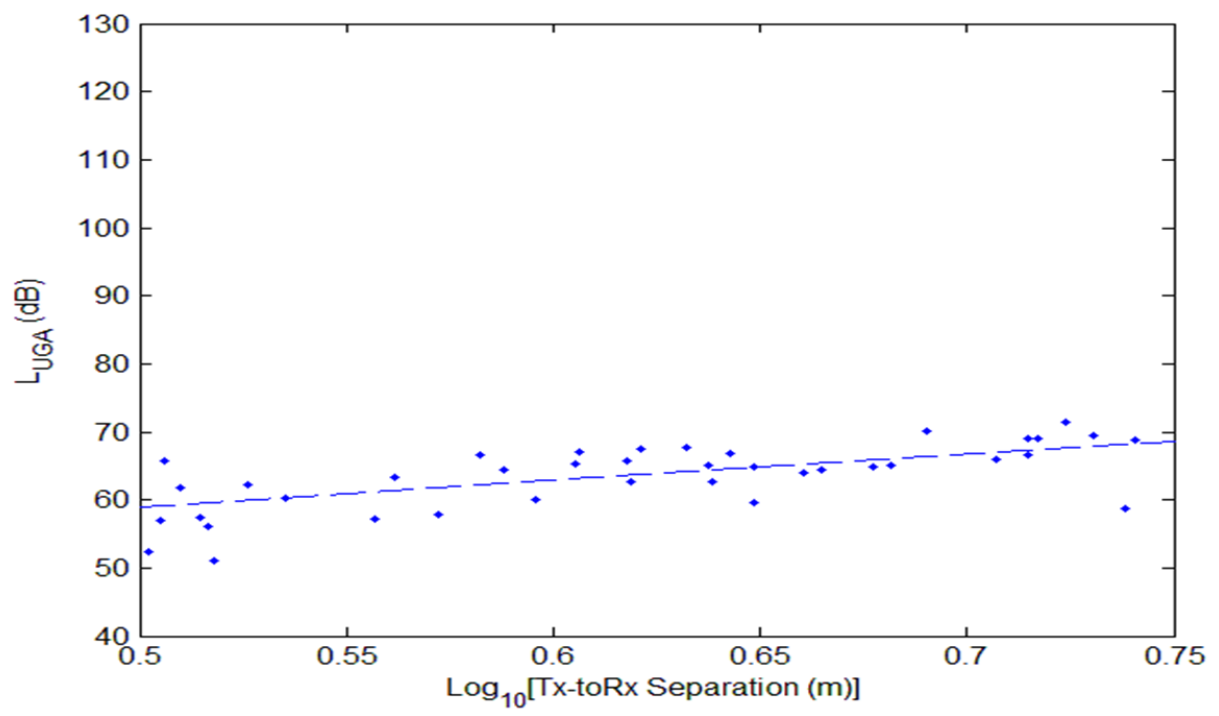

Figure 4.23: Transmission loss measurements and modelling results at $2 \mathrm{GHz}$ 


\subsection{PROPAGATION MEASUREMENTS IN CLUTTER AND CHANNEL MODELLING}

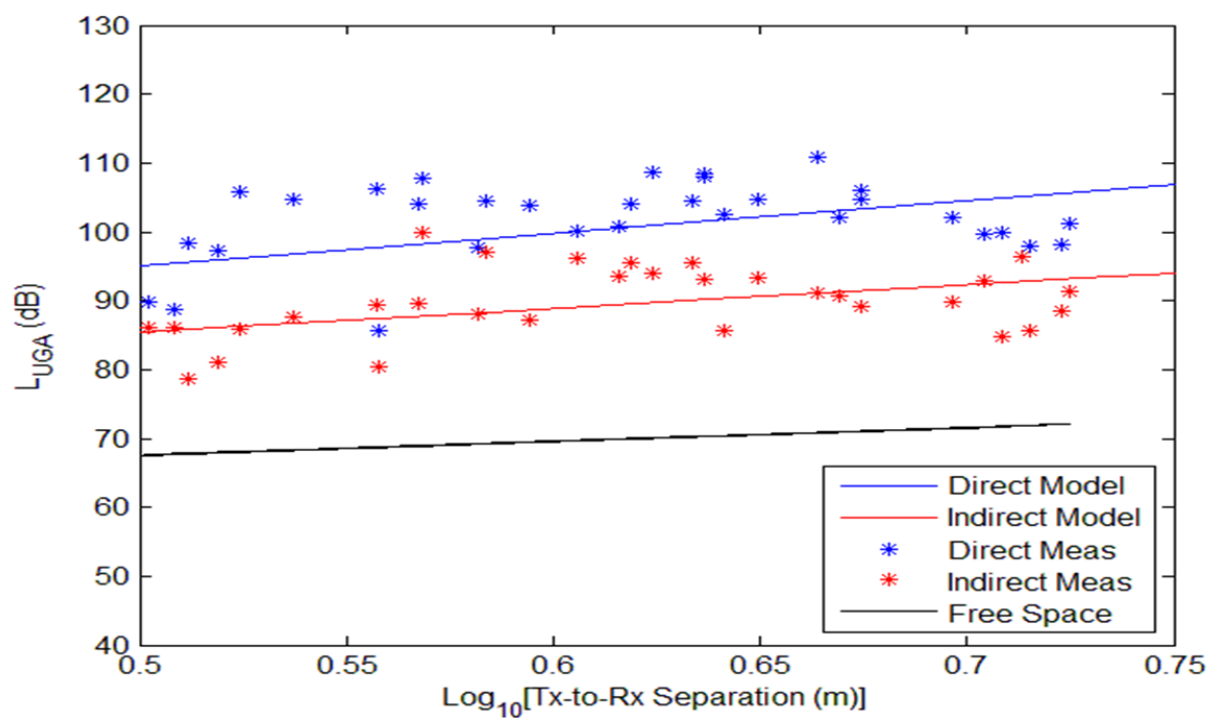

Figure 4.24: Transmission loss measurements and modelling results at $18 \mathrm{GHz}$

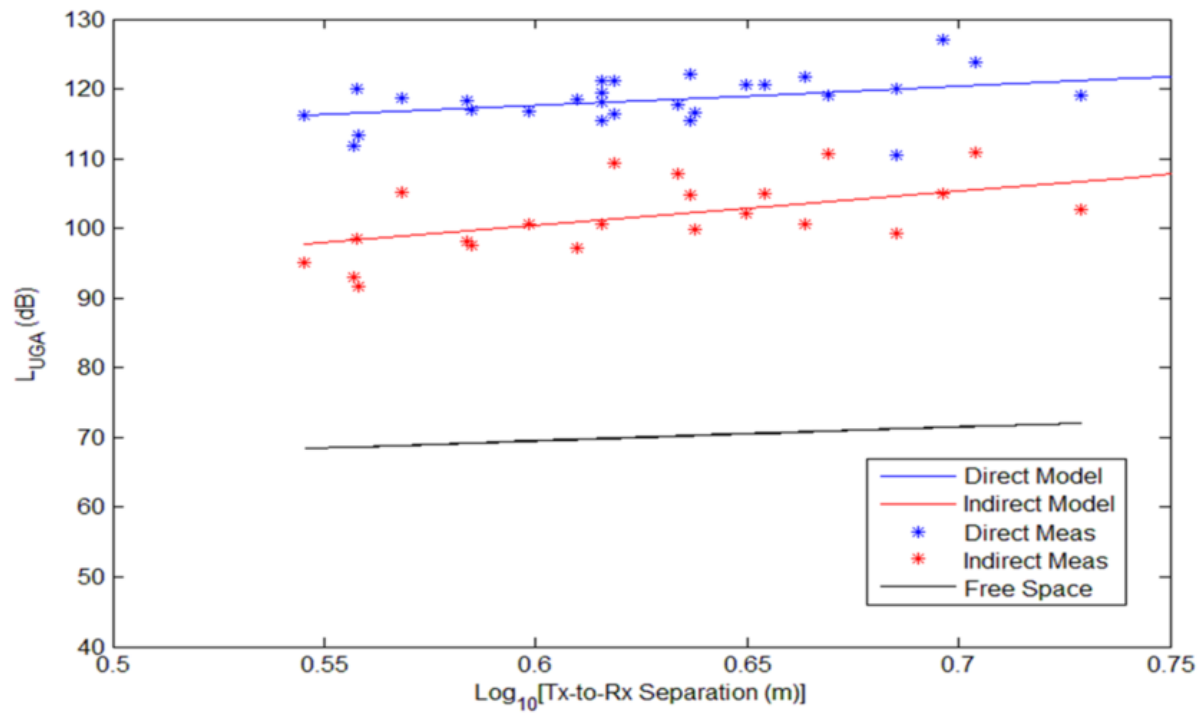

Figure 4.25: Transmission loss measurements and modelling results at $28 \mathrm{GHz}$

Corresponding indirect models for average $L_{U G A}$ in decibels are: 


\subsection{PROPAGATION MEASUREMENTS IN CLUTTER AND}

$$
\begin{aligned}
& L_{U G A}(2 \mathrm{GHz})=58+38 \log _{10}(d / 2.8) \\
& L_{U G A}(18 \mathrm{GHz})=84+33 \log _{10}(d / 2.8) \\
& L_{U G A}(28 \mathrm{GHz})=98+47 \log _{10}(d / 3.5)
\end{aligned}
$$

The shadow loss (at $2 \mathrm{GHz}$ ) and the indirect path loss variation (IPLV) values, in decibels, centred on each model for the average, were verified by means of the onesample Kolmogorov-Smirnov test [31] to be well modelled by a log-normal distribution with zero mean, and a standard deviation that varies with frequency bands. Figures 4.26-4.28 shows the empirical cumulative distribution function ECDF of measured data and the KS test results for a Gaussian model on direct link at $2 \mathrm{GHz}$ and indirect link on $18 \mathrm{GHz}$ and $28 \mathrm{GHz}$ channels with $\mathrm{Rx}$ antenna pointed so as to receive maximum power. The fitting done using 45 independent points in both cases of $2 \mathrm{GHz}$ and $18 \mathrm{GHz}$ channels, and with 22 independent points in case of the $28 \mathrm{GHz}$ channels. In all cases the models could not be rejected with $95 \%$ confidence level. 


\subsection{PROPAGATION MEASUREMENTS IN CLUTTER AND}

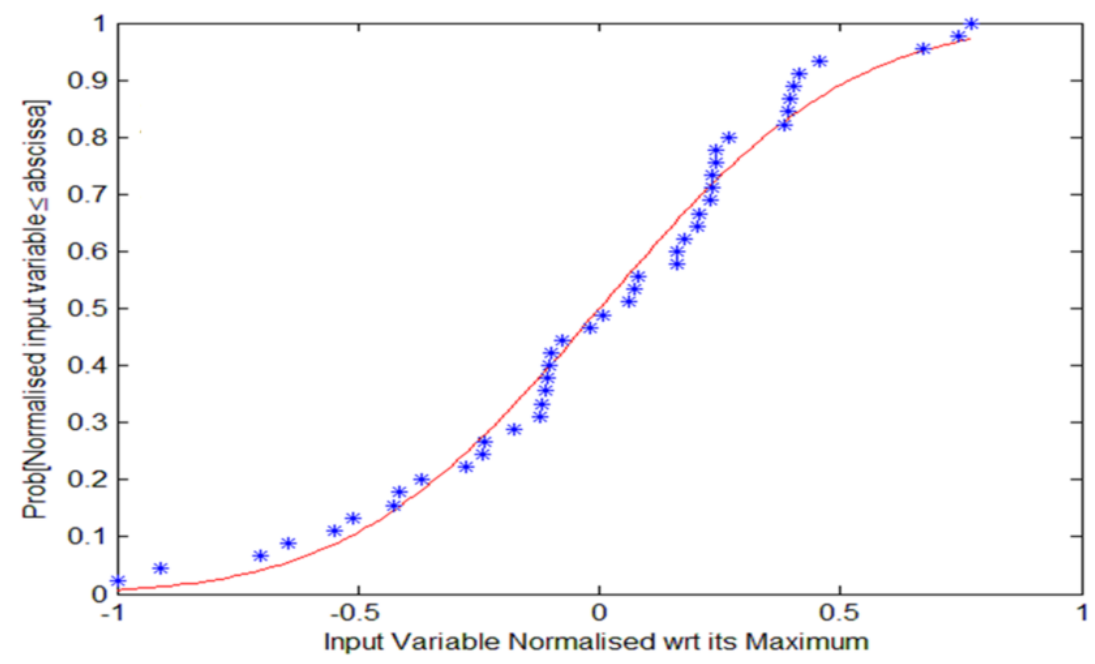

Figure 4.26: ECDF and KS test results for Gaussian model on $2 \mathrm{GHz}$ channels

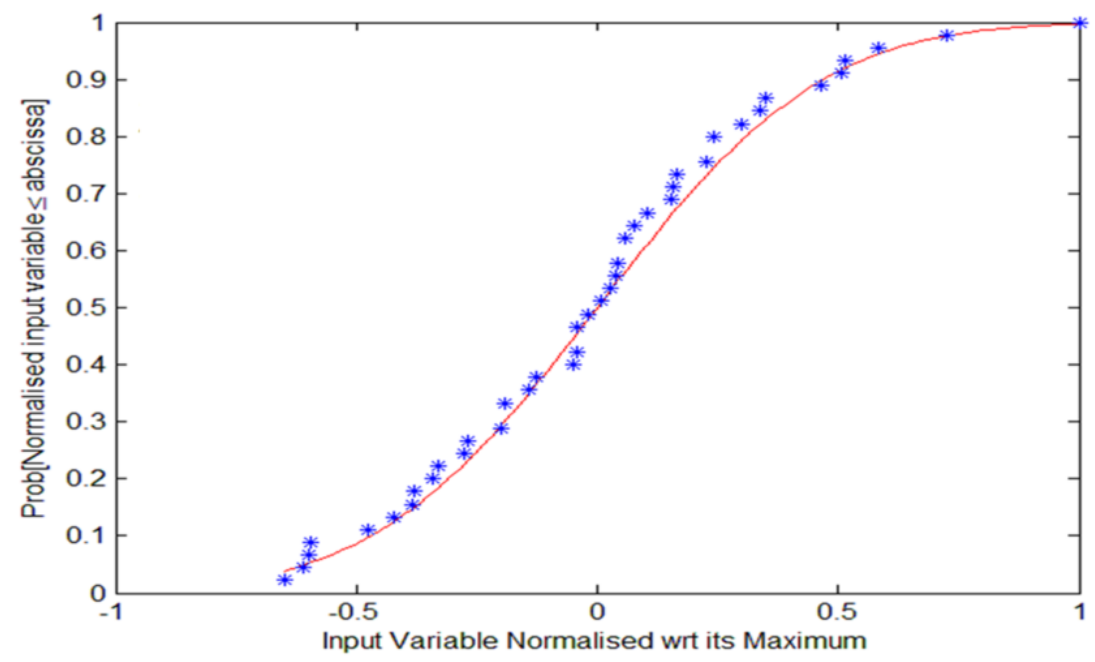

Figure 4.27: ECDF and KS test results for Gaussian model on $18 \mathrm{GHz}$ channels 


\subsection{PROPAGATION MEASUREMENTS IN CLUTTER AND}

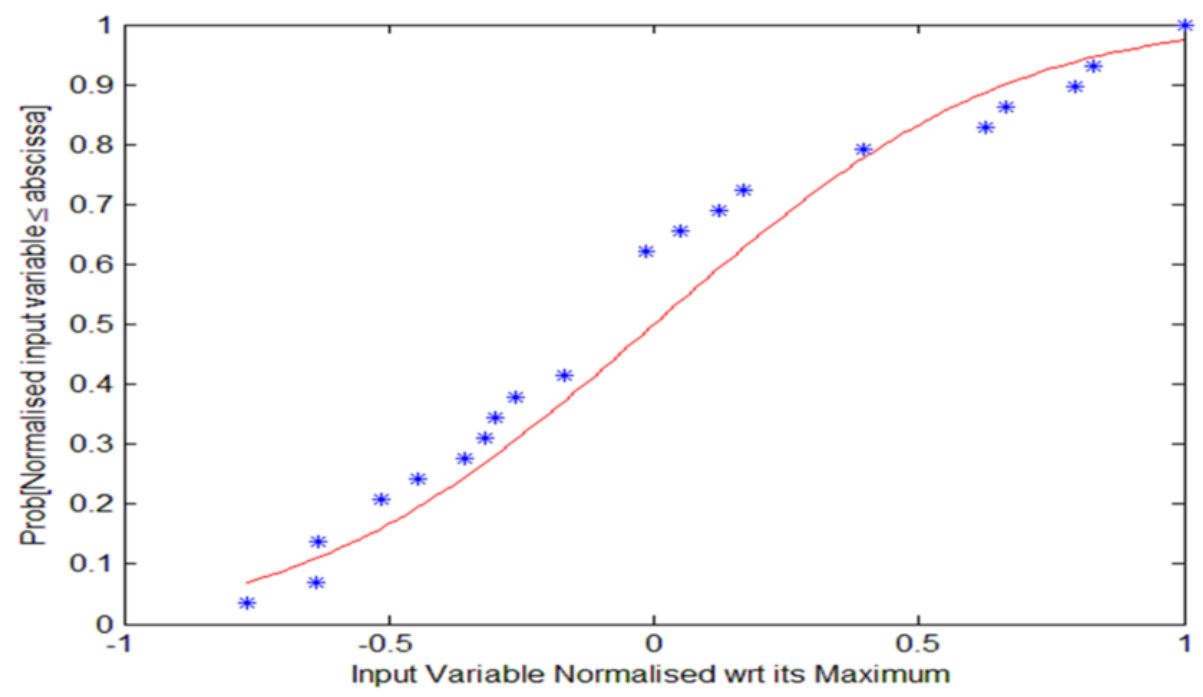

Figure 4.28: ECDF and KS test results for Gaussian model on $28 \mathrm{GHz}$ channels

The resulted shadow and IPLVs models are

$$
\begin{gathered}
\sigma_{S H}(2 \mathrm{GHz}) \cong N(0,3.8 \mathrm{~dB}) \\
\operatorname{IPLV}(18 \mathrm{GHz}) \cong N(0,4.4 \mathrm{~dB}) \\
\operatorname{IPLV}(28 \mathrm{GHz}) \cong N(0,4.2 \mathrm{~dB})
\end{gathered}
$$

where $N(0, \sigma \mathrm{dB})$ represents a normal distribution, with zero mean and standard deviation $\sigma$, in decibels.

\subsubsection{Static RMS Delay}

Radio channel delay spread, represented by its maximum or root-mean-square value, is an important radio channel parameter that determines how significant the effects are of inter-symbol interference due to time dispersion on the performance of radio 


\subsection{PROPAGATION MEASUREMENTS IN CLUTTER AND CHANNEL MODELLING}

communication link. The delay spread of radio channels therefore needs to be quantified from measurements before digital communication systems can be implemented on such channels.

A comparison of static, i.e. single snapshot, RMS delay spreads for the three frequency bands is considered. Figure 4.29 shows static RMS delay spreads calculated after the probabilistic noise rejection thresholding explained in Chapter 3 in all 3 bands.

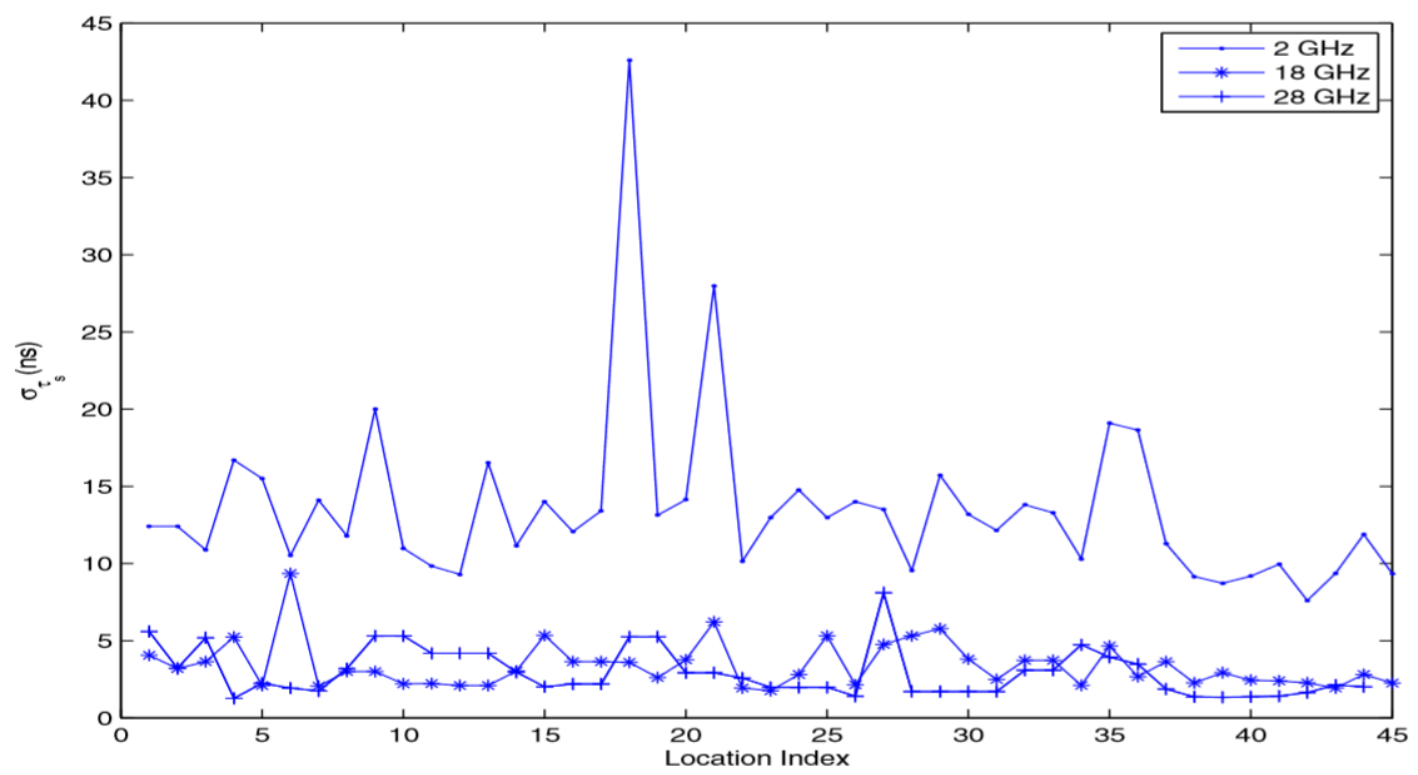

Figure 4.29: Static RMS delay spreads for the 3 frequency bands studied as a function of UE location

It can be seen from Figure 4.29 that the delay spread values for the higher frequency bands are significantly lower. It is considered that this is primarily the result of steering narrow antenna beams in the upper 2 frequency bands at the UE locations to the direction of maximum received power, and is thought to be an additional advantage of such beam steering. 


\subsection{PROPAGATION MEASUREMENTS IN CLUTTER AND CHANNEL MODELLING}

Figure 4.30 is the CCDF of these results. The means, standard deviations, and the median of the RMS delay spreads are depicted in Table 4.6. It can be observed that for indirect path at $18 \mathrm{GHz}$ and $28 \mathrm{GHz}$, RMS delay spreads are lower than they are on direct path in a conventional coverage scenario at $2 \mathrm{GHz}$.

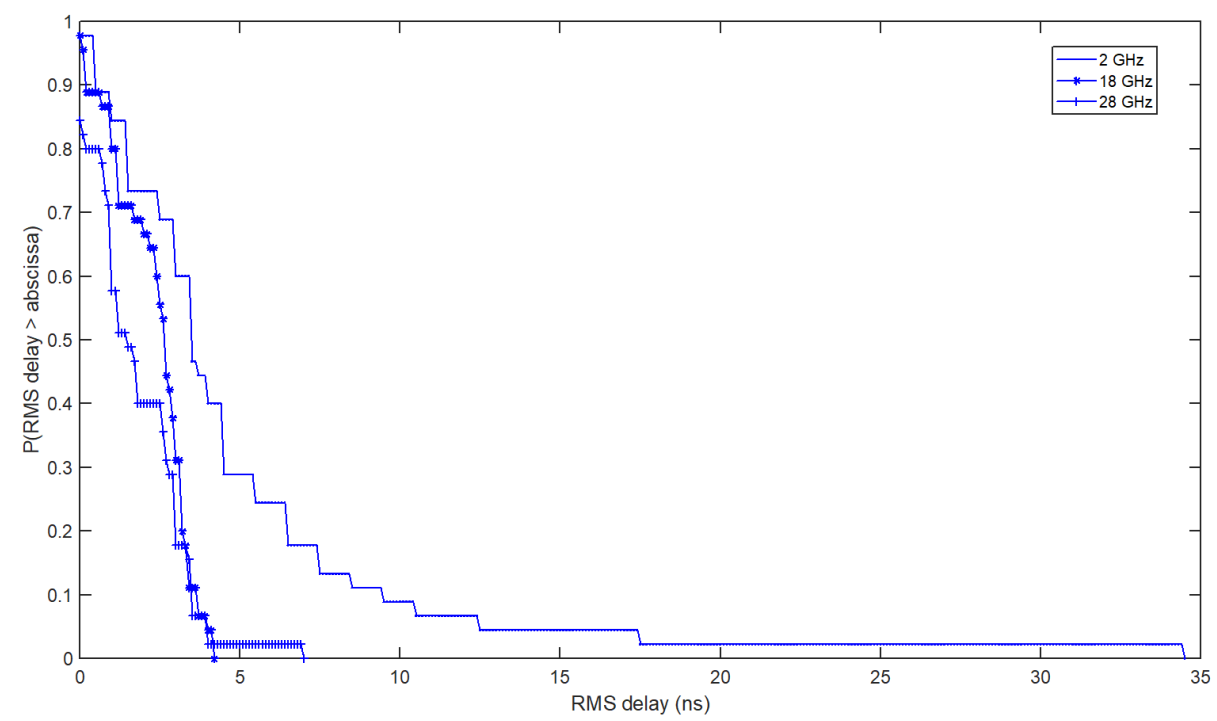

Figure 4.30: Complementary CDFs of RMS delay spreads computed over all measurements

Table 4.6: Statistics of the channel RMS Delay spreads for the three frequency bands

\begin{tabular}{|c||c|c|c|}
\hline $\begin{array}{l}\text { Frequency } \\
{[\mathrm{GHz}]}\end{array}$ & Mean (ns) & Standard deviation & $50_{t h}$ percentile (ns) \\
\hline 2 & 13.4 & 5.6 & 4.1 \\
\hline 18 & 4.1 & 1.1 & 3.0 \\
\hline 28 & 3.8 & 1.5 & 2.1 \\
\hline
\end{tabular}




\subsection{PROPAGATION MEASUREMENTS IN CLUTTER AND CHANNEL MODELLING}

The study of how many different directions in which the received power is strong enough to achieve good reception is vital for the design of an indirect path communication (IPC) system. This is because when a moving object blocks the established indirect-path link between Tx and Rx antennas, the communication link could become unavailable due to high obstruction loss, and therefore steering the receive antenna in alternate directions is necessary to provide redundancy to ensure desired reliability.

Figure 4.31 shows the number of different direction at UE locations in which received power was within $3 \mathrm{~dB}$ of the power received in the direction of the maximum power. As can be seen, on average there are 4 different directions where the $\mathrm{Rx}$ antenna can be steered at to receive a strong signal, this average is decreased to 3 at the $28 \mathrm{GHz}$ band.
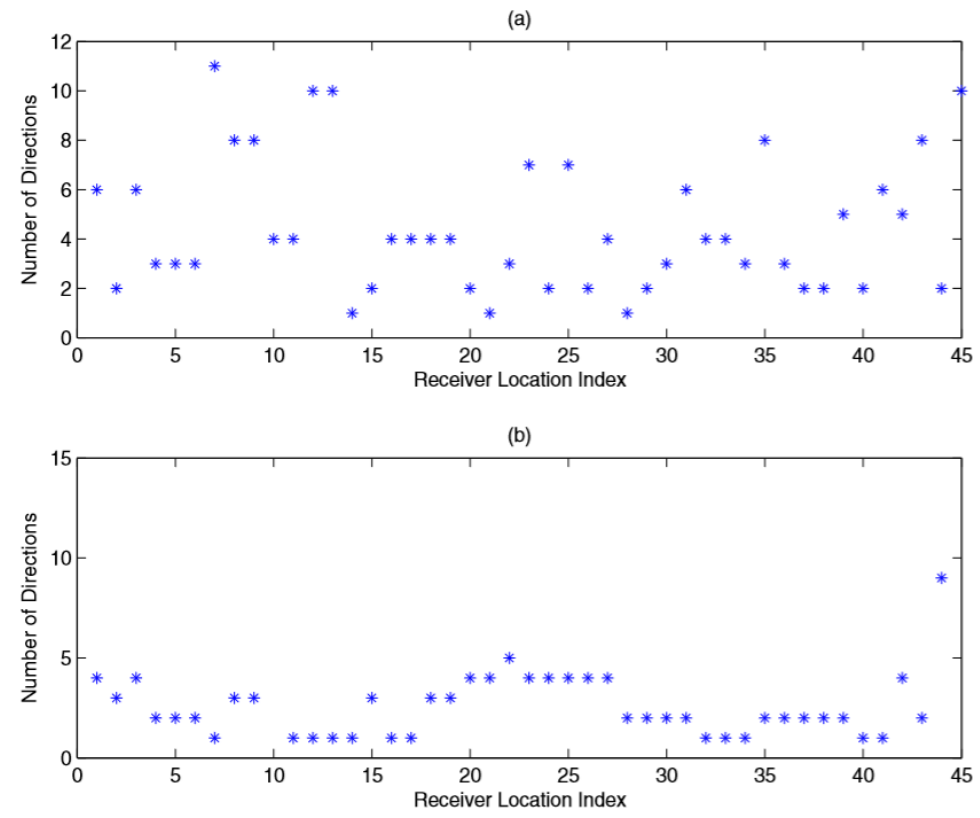

Figure 4.31: The number of different directions at UE locations in which a received power within $3 \mathrm{~dB}$ of the power received in the direction of the maximum can be received: (a) $18 \mathrm{GHz}$, (b) $28 \mathrm{GHz}$ 


\subsection{CHARACTERIZATION OF TEMPORAL VARIATIONS ON INDOOR RADIO CHANNELS}

\subsection{Characterization of Temporal Variations on Indoor Radio Channels}

As noted in Chapter 2, temporal received signal variations on fixed indoor links can be caused either by motion, such as the movement of personnel or machinery, causing changes in multipath conditions surrounding a radio link or by intermittent path obstructions as people walk about. Thus, studying envelop fading characteristics for IPC in an indoor office environment becomes an important aspect to address since it is desired to understand the impact imposed by such variations.

In order to characterize the channel for IPC temporal variations, propagation measurements were carried out using CW equipment at $29.5 \mathrm{GHz}$ as described in chapter 3. Statistical analysis of the CW data was then performed.

\subsubsection{Link Budget Analysis}

For the CW equipment used in propagation measurement $P_{t}=20 \mathrm{dBm}$ and $G_{t}=17$ $\mathrm{dBi}$ therefore the EIRP of the equipment is $37 \mathrm{dBm}$. Free space loss (FSL) at a distance $D$ is given as

$$
F S L_{d B}=-27.55+20 \log _{10}(D)+20 \log _{10}(f)
$$

where $D$ is the distance between Tx and Rx antennas in meters, and $f$ is the frequency in Megahertz. Free space loss for a distance of $1 \mathrm{~m}$ between Tx and Rx antennas at a frequency of $29.5 \mathrm{GHz}$ is $61.8 \mathrm{~dB}$. The power received by an isotropic antenna would therefore be

$$
P_{R_{I}}=E I R P-F S L
$$

The received signal level (RSL) at $1 \mathrm{~m}$ would then be 


\subsection{CHARACTERIZATION OF TEMPORAL VARIATIONS ON INDOOR RADIO CHANNELS}

$$
R S L=P_{R_{I}}+G_{R}-L_{L}
$$

where $G_{R}$ is the receiving antenna gain and it is $20 \mathrm{dBi}$ and $L_{L}$ is the line losses and it is $14 \mathrm{~dB}$. Therefore RSL equals $-18.8 \mathrm{dBm}$. The power at the noise floor $P_{n}$ of the measurement equipment is given by the product of the noise power spectral density $N_{0}$, the receiver IF bandwidth $B_{I F}$ and the noise figure of the receiver, where $N_{0}$ equals $K \times T_{\text {sys }}$, and $K$ equals Boltzmann constant for $T_{\text {sys }}=22^{\circ} \mathrm{c}$, in logarithmic term $K_{T}=-174 \mathrm{dBm}$. Therefore

$$
P_{n}=-174+10 \log _{10} B_{I F}+F
$$

where $F$ is the noise figure of the receiver. For the equipment used $F=2 \mathrm{~dB}$ and the IF bandwidth is $4 \mathrm{MHz}$, making the power at the noise floor equals to $-106 \mathrm{dBm}$. The dynamic ranges available for measurements at Tx-Rx ranges of 2, 4, 6, and 8 meters are listed in Table 4.7 


\subsection{CHARACTERIZATION OF TEMPORAL VARIATIONS ON INDOOR RADIO CHANNELS}

Table 4.7: Link budget of CW equipment for different distances

\begin{tabular}{|l||l|l|l|l|l|}
\hline \multicolumn{1}{|c||}{ Link Budget } & \multicolumn{1}{|c|}{$1 \mathrm{~m}$} & \multicolumn{1}{c|}{$2 \mathrm{~m}$} & \multicolumn{1}{|c|}{$4 \mathrm{~m}$} & \multicolumn{1}{c|}{$6 \mathrm{~m}$} & \multicolumn{1}{c|}{$8 \mathrm{~m}$} \\
\hline EIRP (dBm) & 37 & 37 & 37 & 37 & 37 \\
\hline FSL (dB) & 61.8 & 67.8 & 73.8 & 77.4 & 79.9 \\
\hline P_Ri (dBm) & -24.8 & -30.8 & -36.8 & -40.4 & -42.9 \\
\hline RSL (dBm) & -18.8 & -24.8 & -30.8 & -34.4 & -36.9 \\
\hline P_n (dBm) & -105.9 & -105.9 & -105.9 & -105.9 & -105.9 \\
\hline SNR (dB) & 87.1 & 81.1 & 75 & 71.5 & 69 \\
\hline Dynamic range (dB) & 77.1 & 71.1 & 65 & 61.5 & 59 \\
\hline
\end{tabular}

\subsubsection{Propagation Measurement Scenarios and Procedures}

As discussed earlier, at frequencies above $6 \mathrm{GHz}$, steering narrow beams in a cluttered environment might be required to focus on indirect signals from a particular direction that provides a strong signal. Considering this fact temporal fading in IPC system caused by personnel movement in the area surrounding a UE, which is considered a typical case in an office environment, has been observed and analyzed. The transmitter antenna described in Section 3.2.1 was mounted at the end of the room, at the middle of the end wall, and at the height of $3 \mathrm{~m}$ above floor level, with a down-tilt of $50^{\circ}$. The Rx antenna described in section 3.2.2 was positioned on a PTU at a height of $1.5 \mathrm{~m}$ and the PTU was scanned in azimuth and elevation to find maximum receive power. Once the pointing direction is established, four people moved randomly throughout the environment for 10 mins without braking the indirect path. During the motion the received signal was sampled at a rate of $2 \mathrm{~K}$ samples per seconds. 
This was done for 10 different IPC scenarios and one LoS scenario.

\subsection{Observations}

No fading was observed on Los link even though people were moving in the surrounding environment, however, in IPC scenarios

1. The movement of personnel in the area surrounding an indirect radio link produces signal variation.

2. Initial visual inspection showed that the variations are moderate.

3. The rapidity of fading depends on the speed of movement, slow movement produces slow fading, while fast movement produces fast fading.

4. Fade depth depends on the distance between the indirect path and moving personnel with fading being deeper as the personnel get closer to the path

5. Fading is not a continuous process, and depends on the motion of personnel.

\subsection{Data Analysis}

As mentioned in Section 3.2.2, the log-amp gives a dc output that is proportional to the received power. This dc output was sampled with an 16-bit A/D converter. A calibrated Signal Hound measurement receiver was used to calibrate the correspondence between amplitude of the dc output from the log-amp and the received power in $\mathrm{dBm}$, then the calibration curve was used to translate recorded dc voltages into receive powers in $\mathrm{dBm}$. The calibration curve is shown in Figure 4.32 . 
In order to reduce complexity and processing time during analysis, the data from each link were broken into 6 seconds subintervals.

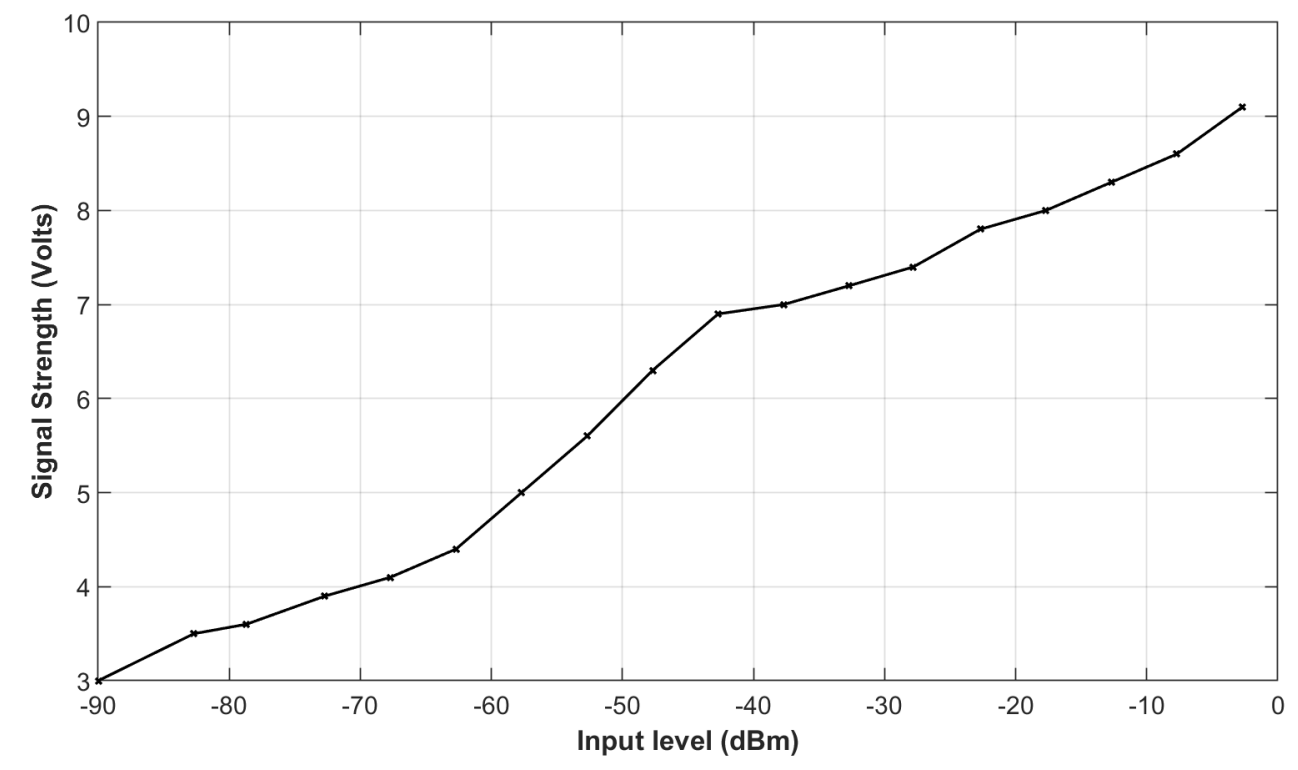

Figure 4.32: Calibration curve for the $\mathrm{CW}$ measurement system

Recorded data were thresholded to select fading and non-fading intervals. For this purpose, the local mean of each half-second subinterval was taken, and thresholding at $3 \mathrm{~dB}$ with respect to the mean was used to identify fading intervals. The data from these intervals were then separated from nonfading data. With the intent of data pooling for better significance in statistical analysis. However, to concatenate identified fading subinterval, it is essential to make sure that they were coming from the same sample distribution. For this purpose data samples from subintervals were tested to ensure a high probability that they were member of the same distribution using the two-sample Kolmogorov-Smirnov test at the 5\% significance level. It is 
important when using the KS test to choose an appropriate critical value. The critical value is a function of the significance level and the number of independent data samples. Therefore, in each subinterval, samples were tested for independence using visual inspection of a scatter plot. Each data set was divided into odd and even sample sequences $\mathrm{x}$ and $\mathrm{y}$, then a scatter plot of $\mathrm{x}$ against $\mathrm{y}$ was plotted. If the scatter was significant, independence was assumed. Otherwise, sample spacing between consecutive $\mathrm{x}$ and consecutive $\mathrm{y}$ values is increased until a good scatter was obtained. Then the size of the resulting data set was used to compute the critical value.

Figure 4.33 shows two scatter plots for two cases, subplot (a) corresponds to small sample spacing where serial dependence is high, and subplot (b) corresponds to a large sample spacing where serial dependence is lower. Sample spacing is adjusted accordingly and the resulted sample size is then used to assign the critical value. The critical value for the distance along the ordinate between the two CDFs for $5 \%$ significance is given in [31] as

$$
D_{n, \alpha}=1.35810 \sqrt{\frac{N+M}{N M}}
$$

where $\mathrm{N}$ and $\mathrm{M}$ are the sample sizes of each data set.

Figure 4.34 shows two ECDFs used in a KS test with 365 samples given a critical value of 0.1007 . Since the maximum difference between the two ECDFs is less than the critical value then the null hypothesis was not rejected. 

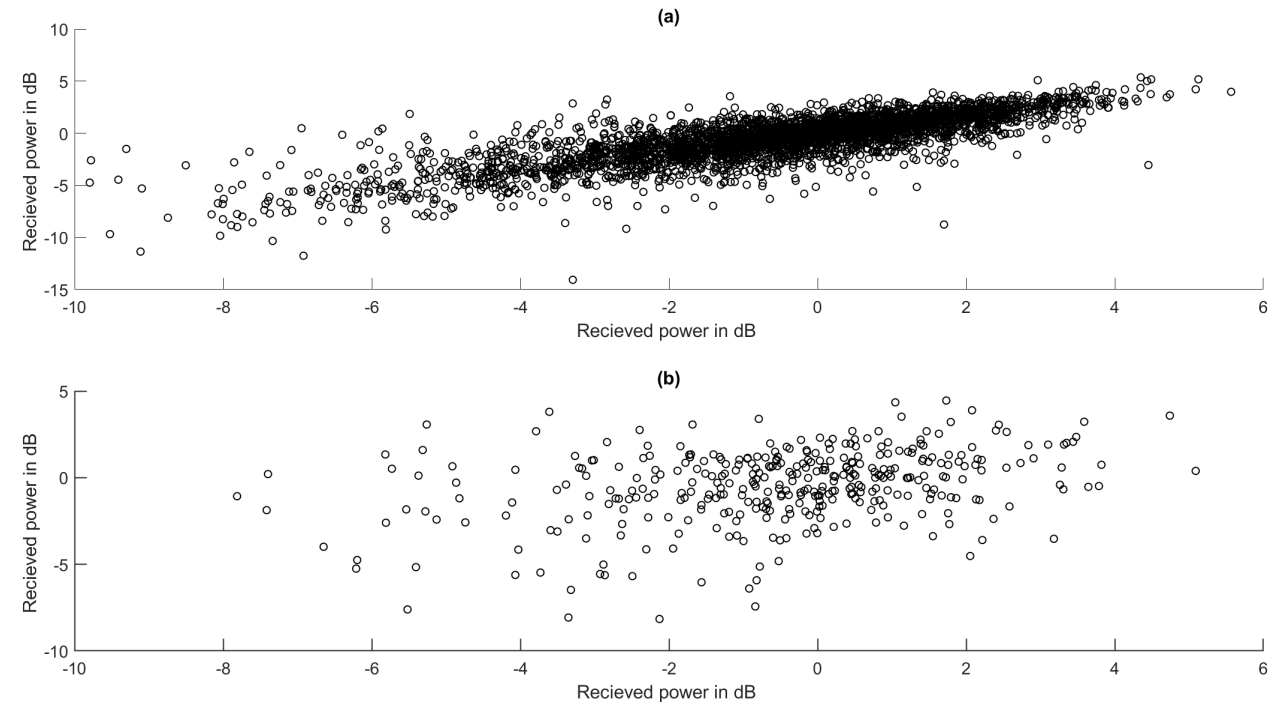

Figure 4.33: Scatter plots (a) for small sample spacing, (b) for adequate sample spacing

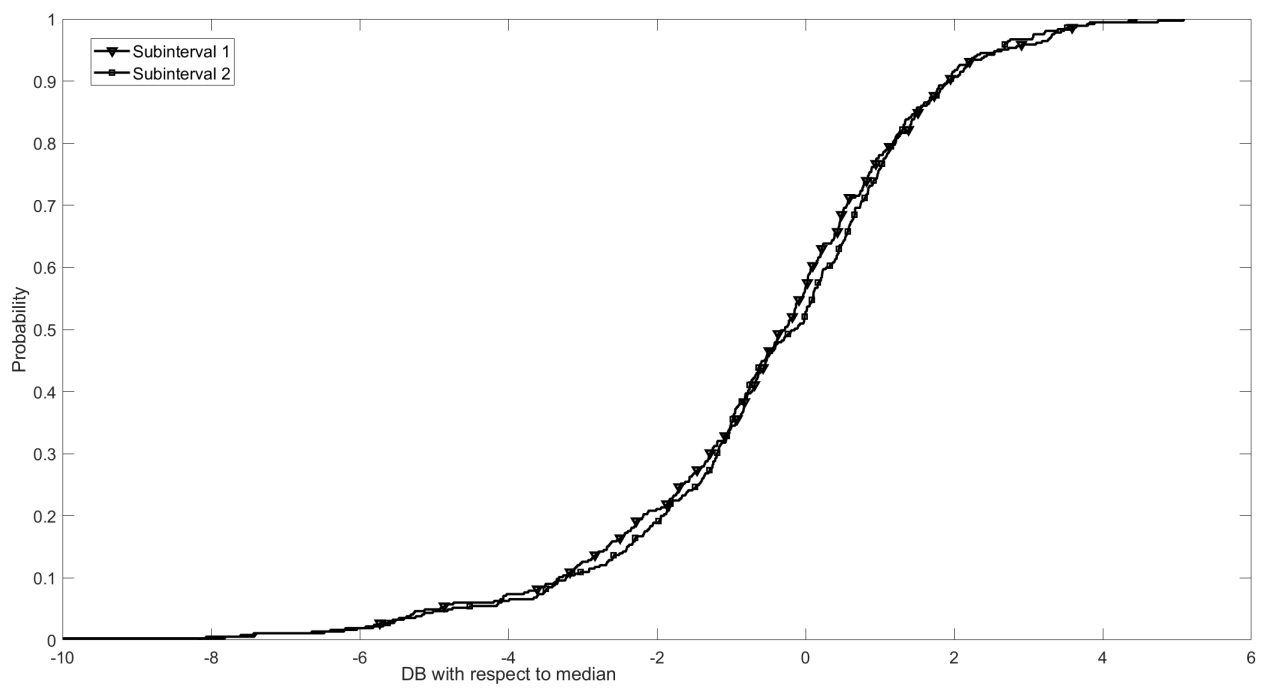

Figure 4.34: ECDFs used in two-sample KS test

In order to find the fluctuation range, maximum and minimum values (including 
outliers) of the receive signal power were calculated for each one of the ten observed links. Figure 4.35 shows the results. The minimum and maximum signal strength observed were -69 and $-36.5 \mathrm{dBm}$, respectively. Dynamic ranges varied between $7 \mathrm{~dB}$ to $30 \mathrm{~dB}$.

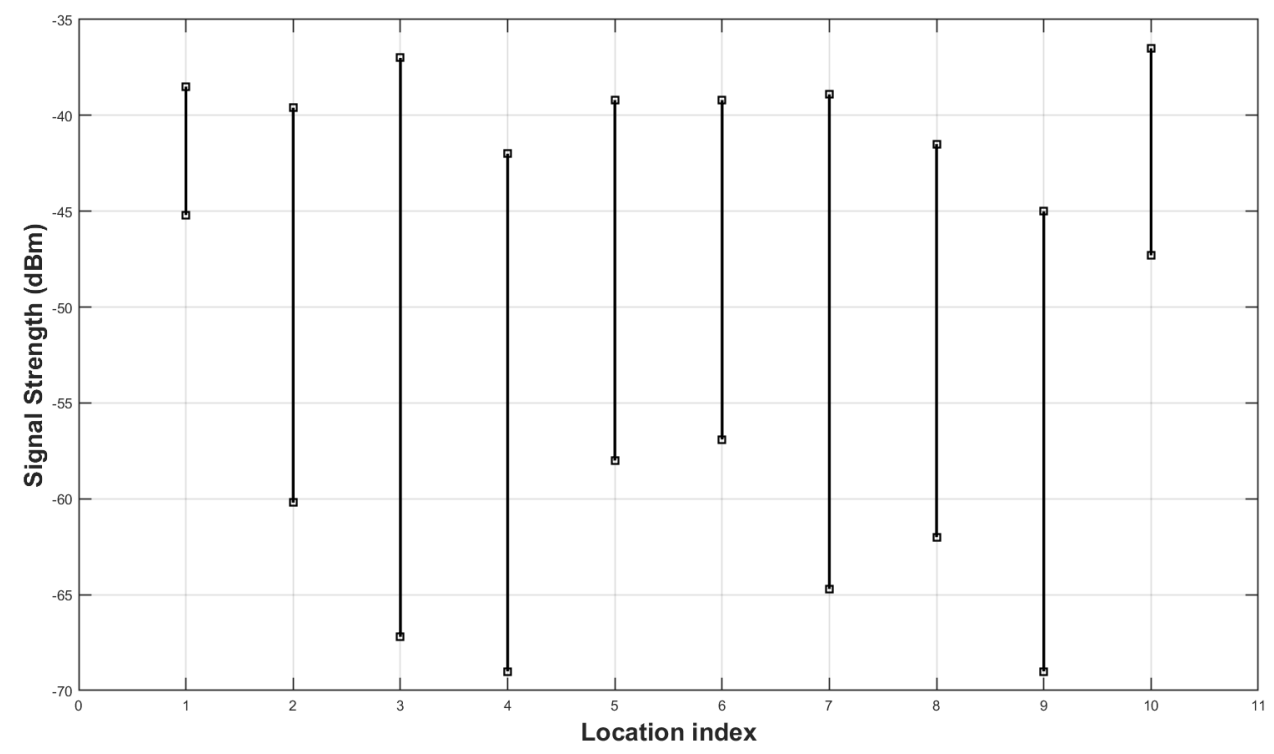

Figure 4.35: Dynamic ranges for identified fading intervals

Typical envelopes of fading for 6-second subintervals are shown in Figures 4.36 and 4.37. As can be seen in the figures, different rates and depths of fading can be observed at different subintervals. 


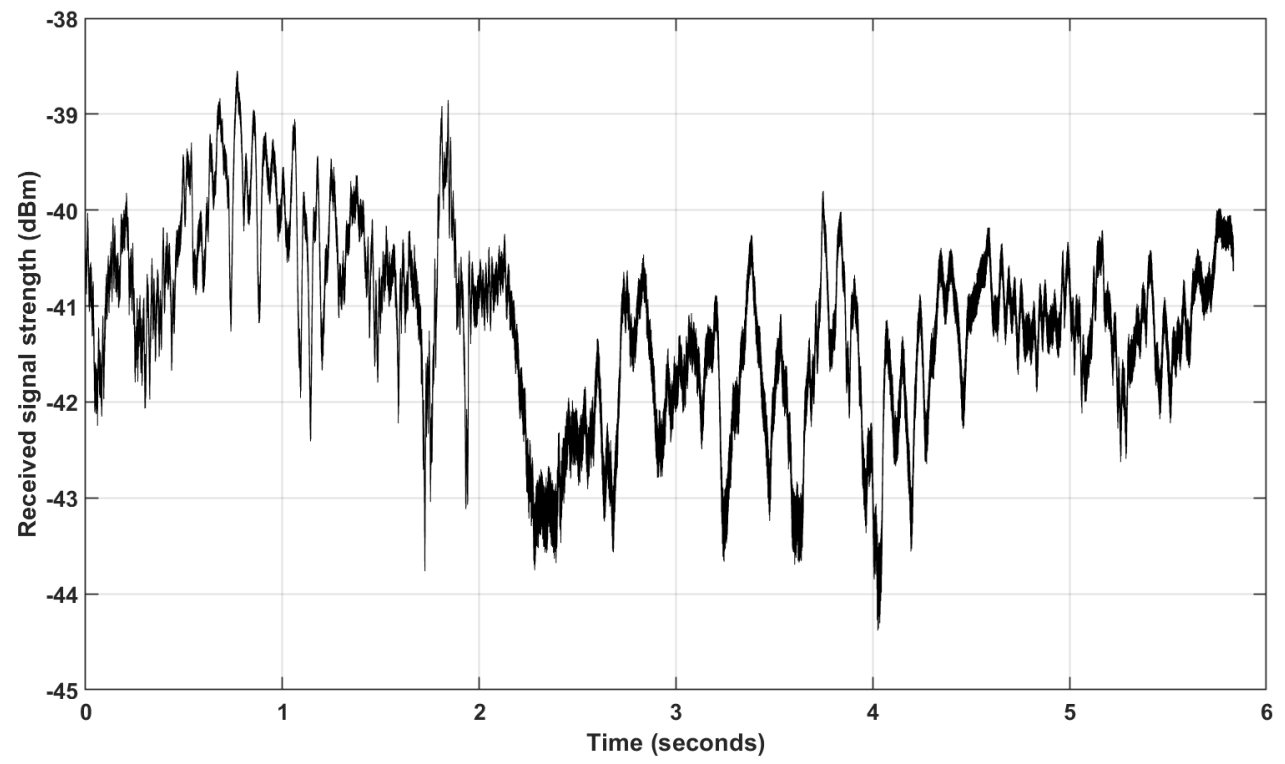

Figure 4.36: An example result of received signal variations over time with dynamic range of $5 \mathrm{~dB}$

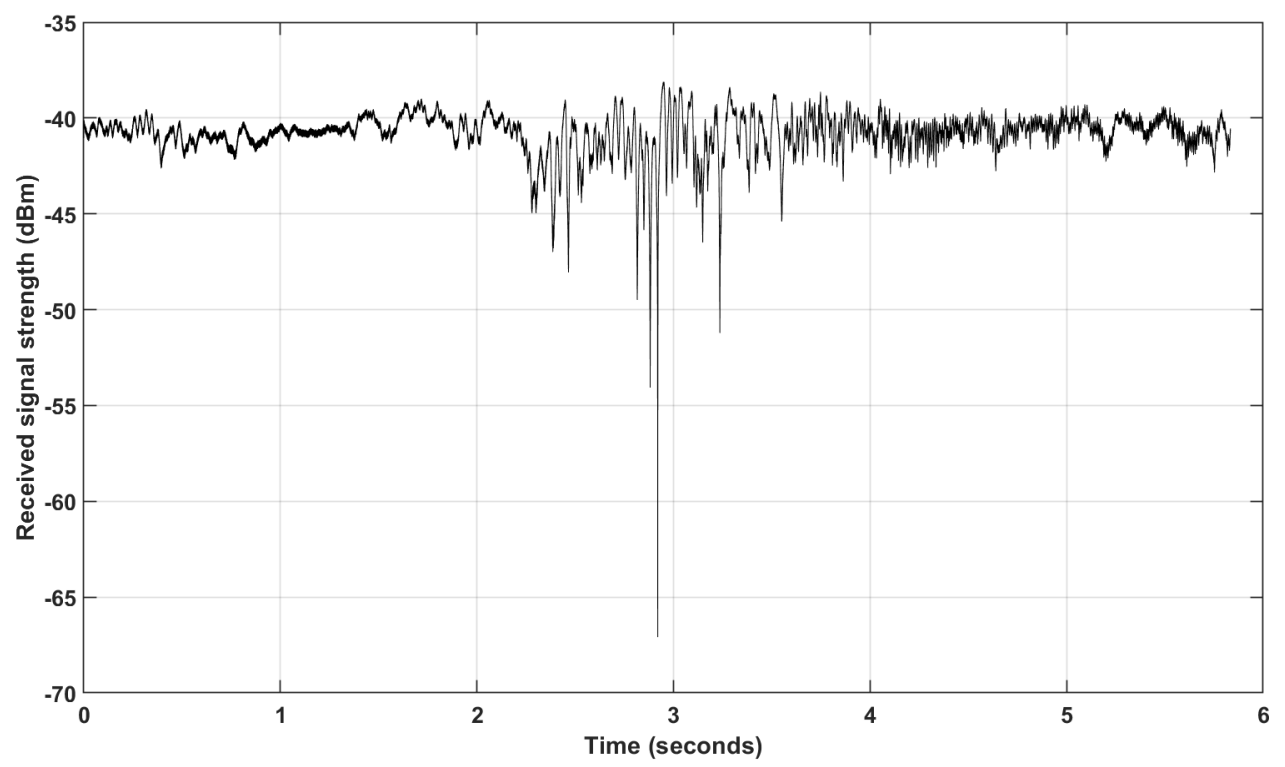

Figure 4.37: An example result of received signal variations over time with dynamic range of $30 \mathrm{~dB}$ 


\subsubsection{Modelling of Envelope Fading Distribution}

The ECDFs of observed samples of received signal fading were compared with Rayleigh and Rician CDFs. Rayleigh and Rician fading are explained in detail in Appendix A.

Goodness of fit analysis using the one-sample Kolmogorov-Smirnov test [32] for the significance level of 0.05 was applied. Once the standard deviation $\sigma$ of envelope variations was estimated, and the specular/random power ratio $(\mathrm{K})$ was estimated using the moment-method [33] for candidate Rayleigh and Rice models, the corresponding CDF models were compared to the ECDFs for each concatenated sequence. The null hypothesis that the models fit the experimental results was not rejected if the maximum distance along the ordinate between the ECDFs and the model being tested $K_{E}$ was less than or equal to a so-called critical value $D_{n, \alpha}$. This critical value depends on both the number of serially-independent samples $\mathrm{N}$ used to estimate each ECDF, and the significance level of the test $\alpha$. That is, the condition required for acceptable modelling can be written as

$$
K_{E}=\operatorname{Max}\left|F_{m}\left(x_{i}\right)-F_{e}\left(x_{i}\right)\right| \leq D_{n, \alpha}
$$

$F_{m}\left(x_{i}\right)$ is the $\mathrm{CDF}$ of the estimated distribution, $F_{e}\left(x_{i}\right)$ is the CDF of the experimental (empirical) distribution, and $D_{n, \alpha}$ is given by $D_{n, \alpha}=1.3581 \sqrt{N}$ for 0.05 significance level with $N>50$.

It was found that $97 \%$ of the identified fading intervals could be modelled using Rician distribution with $\mathrm{K}$ ratios ranging betwenn 6 and $8 \mathrm{~dB}$, and only 3 percent passed the test for both Rayleigh and Rician. The ECDF of the measured data from one subinterval compared with the CDF of Rician distribution with $\mathrm{K}$ factor of 7.4 $\mathrm{dB}$ is shown in Figure 4.38 . 


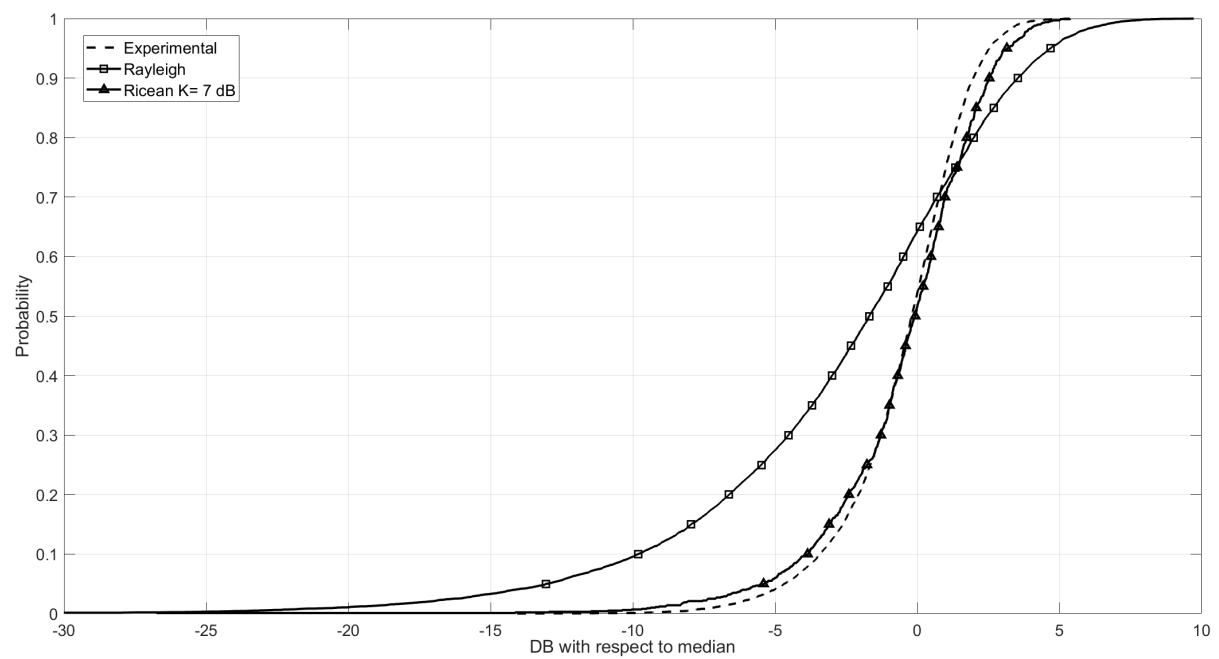

Figure 4.38: ECDF and KS test results for Rayleigh model and Ricean model $\mathrm{K}=7$ $\mathrm{dB}$

The overall result of KS test for the significance of 0.05 , for personnel movement in an indoor modern-indoor office environment is summarized as follows.

- Rician distribution passed for 97 out of 100 subintervals (97\%) with $K$-factors range from 6 to $8 \mathrm{~dB}$.

- Rayleigh distribution passed for only 6 out of 100 subintervals (6\%). 


\section{Chapter 5}

\section{On the Capacity of Multi-User Distributed MIMO Channels}

A distributed antenna system, or DAS, is a network of spatially separated antenna nodes connected to a common source via a transport medium that provides wireless service within a geographic area or structure. A distributed antenna system may be deployed indoors or outdoors. An example illustration of a distributed antenna system is shown in Figure 5.1.

Initial theoretical and experimental investigations suggest that distributed multiantenna systems can mitigate signal attenuation and dramatically increase achievable rates [34], [35], [36]. The potential gains of a distributed MIMO (D-MIMO) system stem from two main factors. First, in a well-designed D-MIMO system, the average distance of each user equipment (UE) to the closest serving antenna is smaller than that in a co-located MIMO system. This implies that, on average, the transmitted signal from a distributed antenna unit (DAU) experiences less transmission loss on its path to any given UE. Also, D-MIMO systems enable macro-diversity to mitigate the effects of shadowing. 
However, when studying D-MIMO system it is essential to consider the fundamental differences from a C-MIMO system, in which, each DAU in D-MIMO has different channel gains since uplink/downlink signals between the user terminals and the different antennas at DAU experience different multipath fading, shadowing and path loss characteristics. Due to these differences, channel modeling and capacity analysis of DASs can not be carried out using existing tools for conventional collocated MIMO systems.

Also, as discussed in previous chapters, it is anticipated that $5 \mathrm{G}$ networks will use carrier frequencies above $6 \mathrm{GHz}$ to provide high data rates for a large number of users. However, higher frequencies experience larger transmission loss and greater diffraction loss in cluttered indoor environments, with the result that conventional outdoor to indoor system or indoor centralized system suffer from a great penetration loss and shadow effects. Despite extensive research effort into evaluating DASs, a propagation-measurement-based calculation of the capacity of multi-user D-MIMO for indoor channels is could not be found in the literature. Thus, it is of practical importance to conduct this study. 


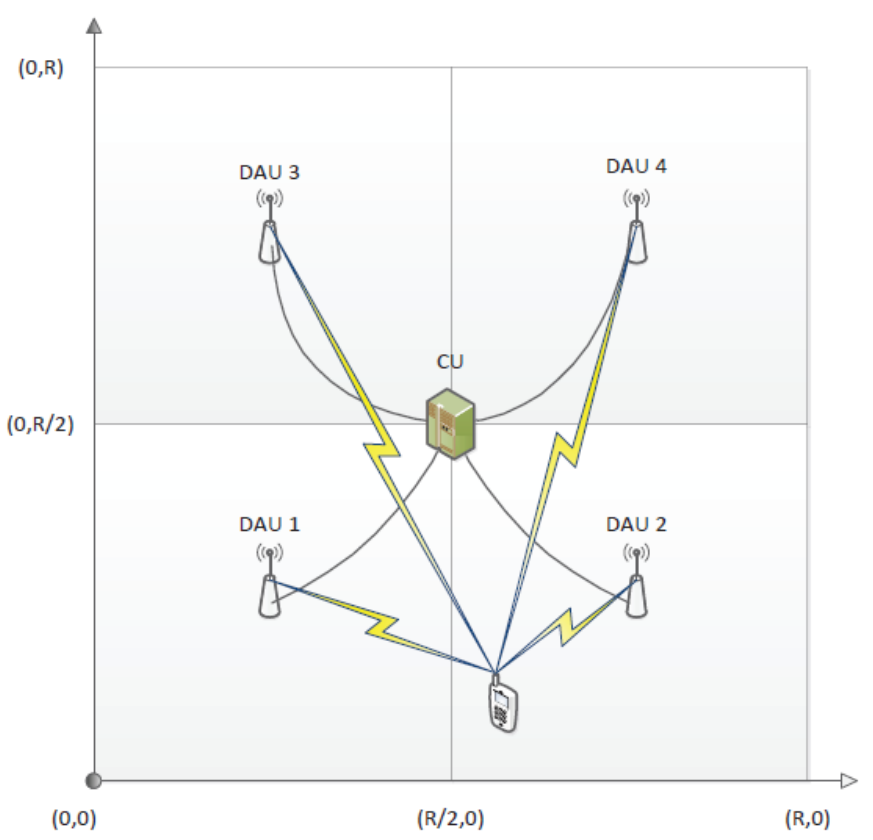

Figure 5.1: Distributed Antenna System model

\subsection{System Model}

In a practical indoor DAS, the number of DAUs and their placement are determined by the specific environment and user distribution. For the cluttered indoor environment where propagation measurements were made, 4 DAUs are considered, one mounted in the middle of each wall, at a height of $4 \mathrm{~m}$ above floor level, and above a suspended ceiling at $3 \mathrm{~m}$ height. Also, it is assumed that each DAU antenna has characteristics similar to those of the Tx antennas used in the experiments. It is further assumed that there are 4 UEs and that at $2 \mathrm{GHz}$, the UEs have omnidirectional antennas; whereas and in the upper bands, each UE has an antenna subsystem with an ability to steer 4 independent beams with beamwidths of 10 degrees. This is considered achievable with the small size of antenna arrays at these frequencies. Thus in total 
16 links exist in the subsystem, i.e., $\mathrm{L}=16 . h_{l}$ is used to denote the channel response matrix of the $l_{t h}$ link. Then the DAS channel matrix $\mathbf{H}$ becomes

$$
\mathbf{H}=\left[\begin{array}{cccc}
h_{1,1} & h_{1,2} & h_{1,3} & h_{1,4} \\
h_{2,1} & h_{2,2} & h_{2,3} & h_{2,4} \\
h_{3,1} & h_{3,2} & h_{3,3} & h_{3,4} \\
h_{4,1} & h_{4,2} & h_{4,3} & h_{4,4}
\end{array}\right]=\left[\mathbf{h}_{1} \mathbf{h}_{2} \mathbf{h}_{3} \mathbf{h}_{4}\right]
$$

Consider the downlink of this DAS, in which all DAUs transmit signals to the users simultaneously. The received signals at the UEs can be expressed as

$$
\mathbf{Y}=\mathbf{H x}+\mathbf{n}
$$

where $\mathbf{x}=\left[\left(x_{1}\right)^{T}\left(x_{2}\right)^{T}\left(x_{3}\right)^{T}\left(x_{4}\right)^{T}\right]^{T}$ is the transmit signal vector and $\mathbf{n}=\left[n_{1} n_{2} n_{3} n_{4}\right]$ denotes the complex Gaussian noise, i.e. $n_{l} \sim C\left(0, \sigma^{2}\right)$. The transmit power of the $l_{t h} \mathrm{DAU}$ is $P_{l}$, and $P_{t}=\sum_{l=1}^{4} P_{l}$ is the total transmit power. In the narrow band case $\mathbf{H}$ can be modelled as

$$
\mathbf{H}=\mathbf{L}_{P}^{-1} \alpha_{S} \alpha_{S V} \boldsymbol{\alpha}_{T F}
$$

where $\mathbf{L}_{P}$ is a matrix representing propagation losses ${ }^{1}, \boldsymbol{\alpha}_{S}$ is a matrix of multiplication factors representing the shadowing effects at $2 \mathrm{GHz}$ or IPLVs at the higher frequencies, $\boldsymbol{\alpha}_{S V}$ and $\boldsymbol{\alpha}_{T \boldsymbol{F}}$ are matrices of multiplication factors representing the spatial variations and the small-scale (temporal) fading, respectively. To evaluate system capacities in this scenario, the the propagation models reported in Chapter 4 and given in Eq (4.4

\footnotetext{
${ }^{1}$ In previous chapters this was recognised to be as transmission loss between unity gain antennas often called just transmission loss
} 
- 4.9) were employed to calculate elements in the matrices $\mathbf{L}_{P}, \boldsymbol{\alpha}_{S}$ link loss from each DAU to randomly chosen UE locations in 1000 uniformly distributed (over space) channel realizations.

To account for spatial variations within the local area at distance $d, \boldsymbol{\alpha}_{\boldsymbol{S V}}$ is generated according to Rayleigh distribution at $2 \mathrm{GHz}$ where omnidirectional $\mathrm{Rx}$ antennas are assumed, and according to Ricean distribution with K-factor equals to $7 \mathrm{~dB}$ at $18 \mathrm{GHz}$ and $28 \mathrm{GHz}$. The choice of Ricean distributions for the spatial variations at the two upper bands agrees with the results obtained from $28 \mathrm{GHz}$ and $73 \mathrm{GHz}$ small-scale spatial statistics presented in [37]. The possible physical reason for such distributions is the present of specular reflection in the received signal. The temporal fading term $\boldsymbol{\alpha}_{\boldsymbol{T} \boldsymbol{F}}$ is simulated with Rayleigh distribution at $2 \mathrm{GHz}$ and with Ricean distribution at $18 \mathrm{GHz}$ and $28 \mathrm{GHz}$ with $\mathrm{K}$-factor equals to $7 \mathrm{~dB}$, according to aforementioned results from Chapter 4. More detail on how Rayleigh and Ricean models were generated for both spatial variation and temporal fading are presented in Appendix B. Also Appendix B presents the method of introducing cross correlation and burstiness into Rayleigh and Ricean models.

\subsubsection{Capacity of MIMO Channels}

In a fading environment, the channel matrix is random, hence for each channel state the mutual information is different $[38]^{2}$ and is given by

$$
I(\mathbf{H})=\log \operatorname{det}\left[I+\frac{1}{\sigma_{n}^{2}} \mathbf{H Q H}^{+}\right]
$$

where det is the determinant of [.], $\sigma_{n}^{2}$ is the noise power at each antenna in MIMO

\footnotetext{
${ }^{2}$ In this chapter, the log is to base 2 unless otherwise specied
} 
system, $I$ is the identity matrix, and $\mathbf{H}^{+}$is the transpose conjugate of $\mathbf{H}, \mathbf{Q}$ is the input covariance matrix of the transmit signal.

The channel capacity depends on the availability of channel state information (CSI) at the transmitter and the receiver, and the channel statistics. If the CSI is available at the transmitter and the receiver, then a well-known result for randomly varying MIMO channel is that the capacity achieved for each channel state, effectively representing a deterministic channel [39], is given by

$$
C=\max _{t r_{Q}} \log \operatorname{det}\left[I+\frac{1}{\sigma_{n}^{2}} \mathbf{H Q H}^{+}\right]
$$

However, if only perfect CSIR is assumed, equal power allocation to each transmit antenna is optimal. i.e. $E\left\{X X^{\dagger}\right\}=P_{I}$. In this case, the covariance matrix $\mathbf{Q}$ is known, and is given by

$$
\mathrm{Q}=\frac{P_{T}}{\sigma_{n}^{2} m} I_{t}
$$

obviating the maximization. Then

$$
C=\log \operatorname{det}\left(I+\frac{P_{T}}{\sigma_{n}^{2} m} \mathbf{H H}^{+}\right)
$$

where $P_{T}$ is the transmit power, $\sigma_{n}$ is the noise power at each receive antennas, and $m$ is the number of transmit antennas.

When the channel realization is chosen randomly and remains fixed for the duration of transmission, the average mutual information does not correspond to an achievable capacity since there is always a non-zero probability that the channel realization would not support any positive capacity, so the rate of reliable transmission 


\subsection{SIMULATION PROCESS OF MULTI-USER D-MIMO SYSTEM CAPACITY}

is zero and the notion of outage is introduced. The outage capacity of a MIMO random and non-ergodic channel is defined as the largest rate such that the information outage probability is less than $\epsilon$, i.e.,

$$
C_{\epsilon}=\sup \left\{R: P_{r}(I(\mathbf{H}<R) \leq \epsilon)\right\}
$$

\subsection{Simulation Process of Multi-User D-MIMO System Capacity}

A flow chart illustrating the simulation process for a multi-user system is shown in Figure 5.2.

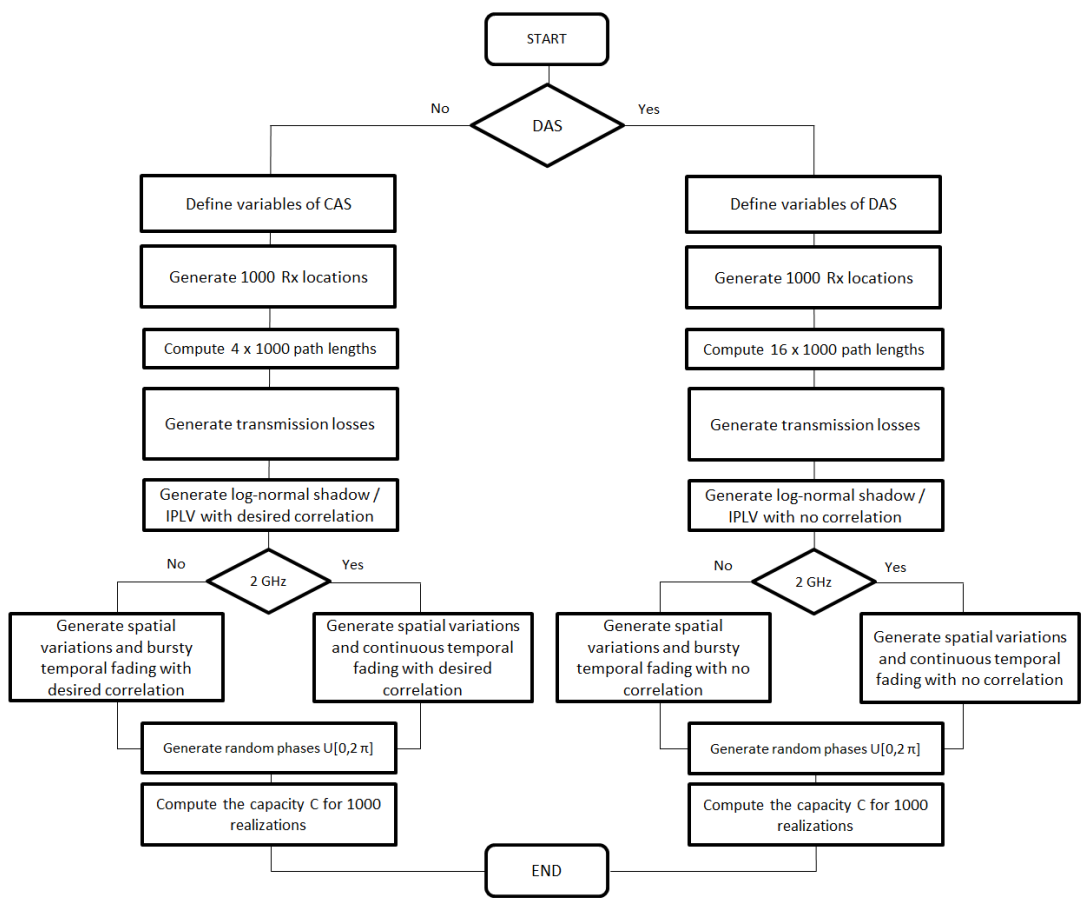

Figure 5.2: A flow chart illustrating the system capacity simulation steps

As can be seen, either for DAS or CAS, the process starts by defining variables such as Tx antenna locations, antenna gains, the centre frequency, etc. The next step 


\subsection{SIMULATION PROCESS OF MULTI-USER D-MIMO SYSTEM} CAPACITY

is to generate the UE locations by generating uniformly distributed random variables for $\mathrm{x}, \mathrm{y}$ coordinates. The same height of $1.5 \mathrm{~m}$ for all users is assumed. Also, each user is assumed to have the ability to steer four beams, therefore, a total of 16 path links results, each with a different path length (in case of DAS). Each path length $D_{l}$ can be computed as

$$
D_{l}=\sqrt{\left(X_{t}-X_{r}\right)^{2}+\left(Y_{t}-Y_{r}\right)^{2}+\left(Z_{t}-Z_{r}\right)^{2}}
$$

where $X_{t}, Y_{t}, Z_{t}$ and $X_{r}, Y_{r}, Z_{r}$ are the spatial coordinates of the Tx and Rx antennas, respectively. Based on these path lengths, link losses are calculated using the measurement-derived propagation loss models. The elements of $\boldsymbol{\alpha}_{S}$ are then calculated from a zero mean log-normal distribution, with standard deviation derived from the shadow loss or IPLV models as applicable. One-sample KS test was applied each time a shadow or IPLV values are generated to ensure the conformance with zero mean log-normal distribution. Example result of KS test that could not been rejected at 95\% confidence level is shown in Figure 5.3. 


\subsection{SIMULATION PROCESS OF MULTI-USER D-MIMO SYSTEM}

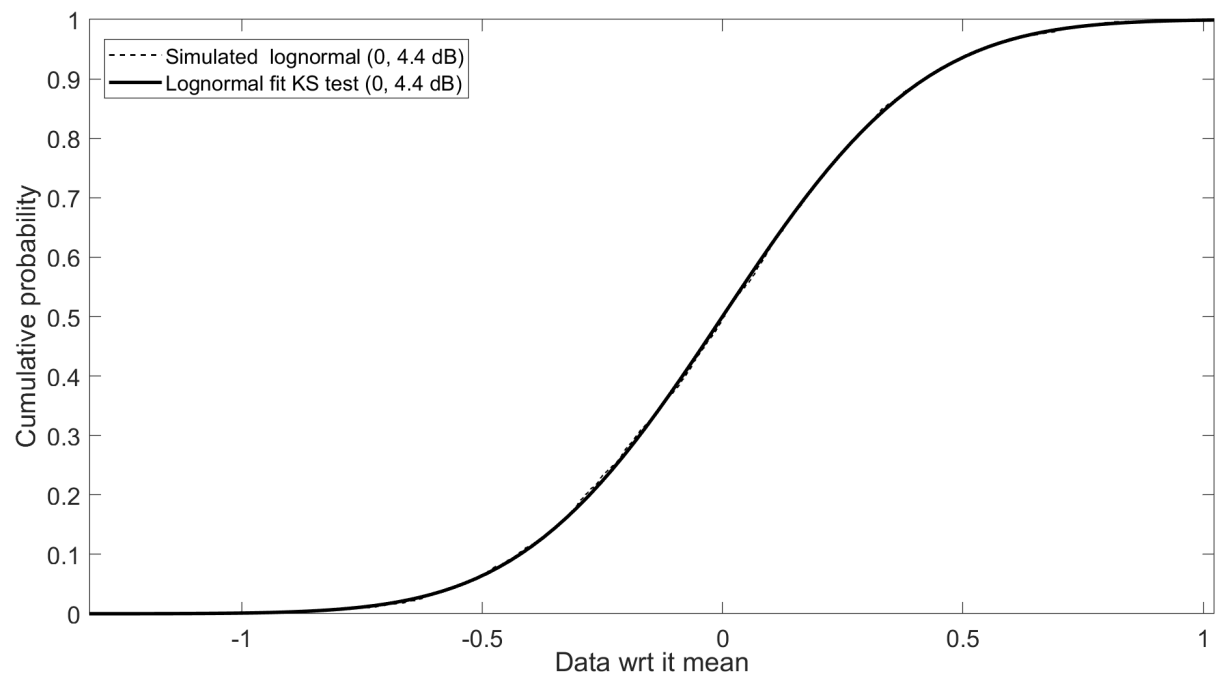

Figure 5.3: ECDF and KS test results for log-normal distribution with 0 mean and $4.4 \mathrm{~dB}$ standard deviation

Data used in the KS test have also been checked for independence through scatter plots before conducting the test, example result of a scatter plot is shown in Figure 5.4. As can be seen the data points are well scattered and by visual inspection the data were assumed to be independent. 


\subsection{SIMULATION PROCESS OF MULTI-USER D-MIMO SYSTEM}

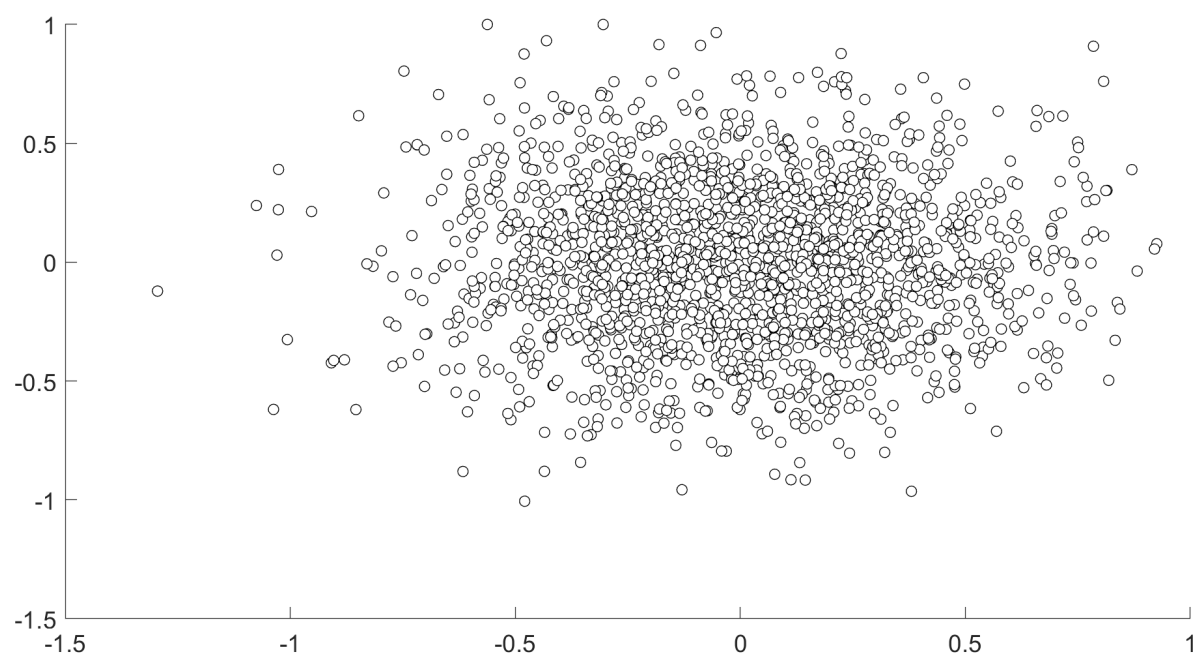

Figure 5.4: A scatter plot showing an approximate independence required for the KS-test

Multiplication factors to account for small-scale spatial variations and small-scale fading are then generated according to Rayleigh or Ricean distributions. For verification, the one-sample KS test was also applied using independent data which have been checked through scatter plots. Figure 5.5 shows KS test example result for Rayleigh, Ricean with $\mathrm{K}=8 \mathrm{~dB}$, and Ricean with $\mathrm{K}=20 \mathrm{~dB}$ all of which could not be rejected at $95 \%$ confidence level. A scatter plot showing approximate independence in data used to perform the KS test is shown in Figure 5.6. 


\subsection{SIMULATION PROCESS OF MULTI-USER D-MIMO SYSTEM}

CAPACITY

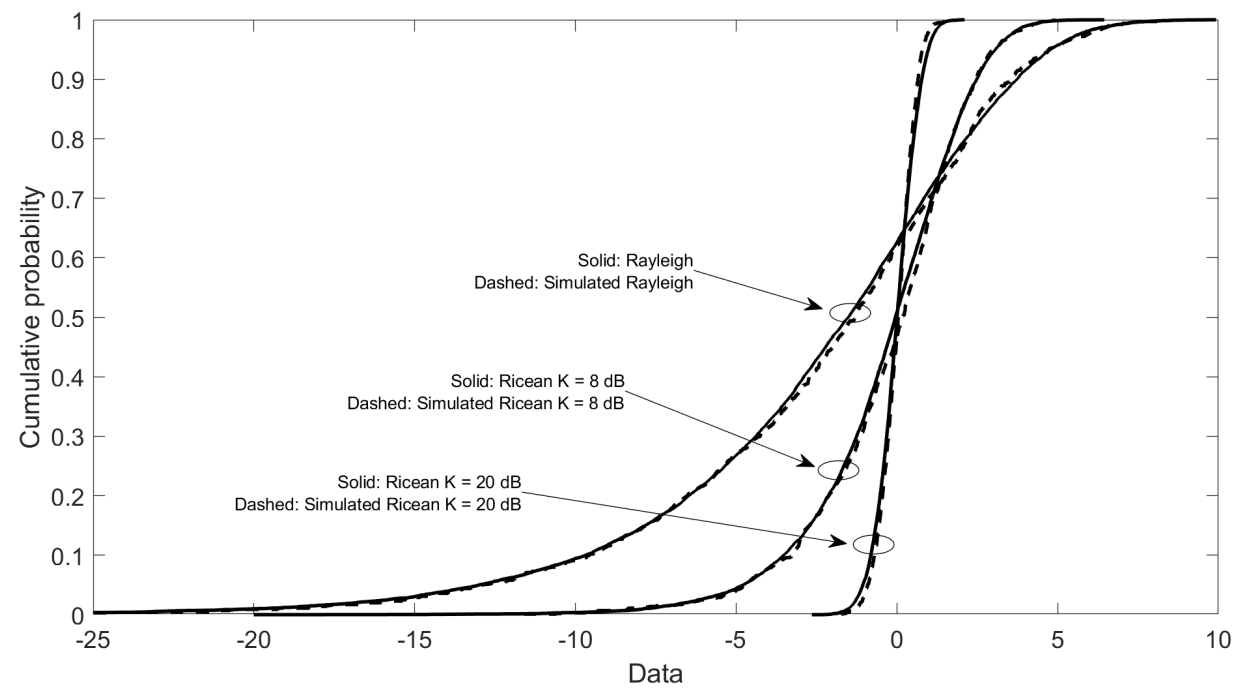

Figure 5.5: A plot showing ECDFs for simulated data and CDFs models for Rayleigh, Rice $\mathrm{K}=8 \mathrm{~dB}$, Rice $\mathrm{K}=20 \mathrm{~dB}$

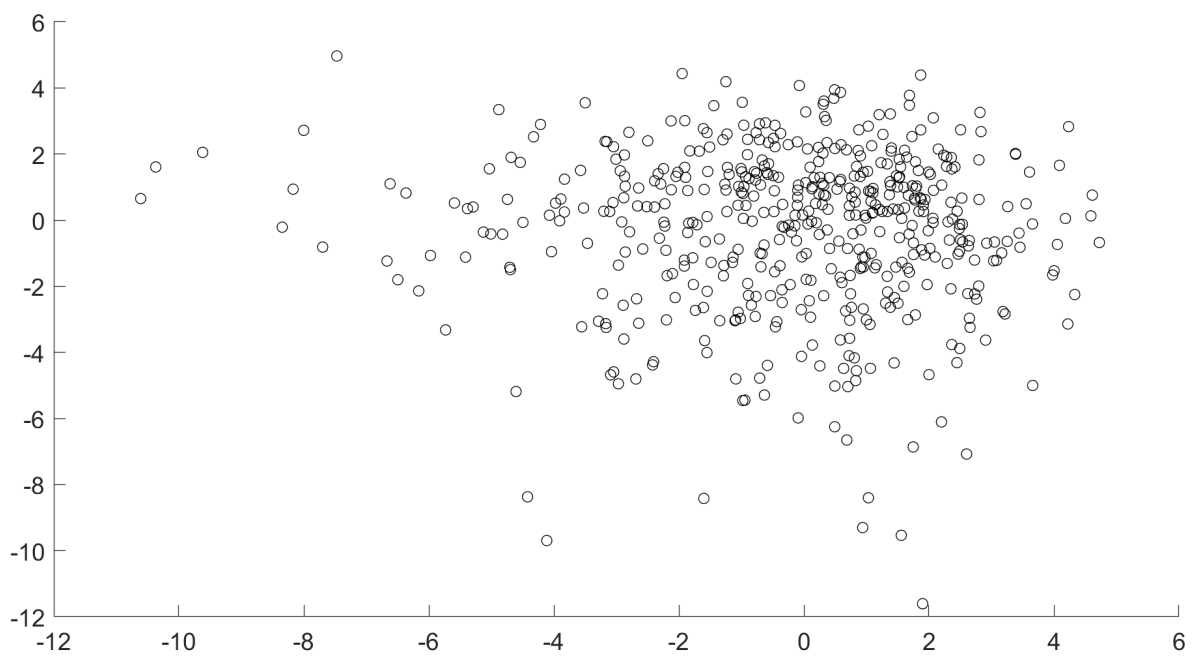

Figure 5.6: A scatter plot showing approximate independence required for the KS-test

The SSSpVs and the temporal fading on the $16_{t h}$ links were simulated by generating 16 Rayleigh envelopes with specific cross correlation coefficient values, and were 
modified, when needed, to generate 16 Ricean envelopes with specific cross correlation values and specific deterministic/random signal component power ratios $(\mathrm{K})$. Scatter plots are a good tool for the visualization of cross correlation. Figure 5.7 shows two example results where (a) shows a comparison of two uncorrelated Rayleigh envelope sample series and (b) shows a comparison of two Rayleigh envelope sample series with $50 \%$ correlation.
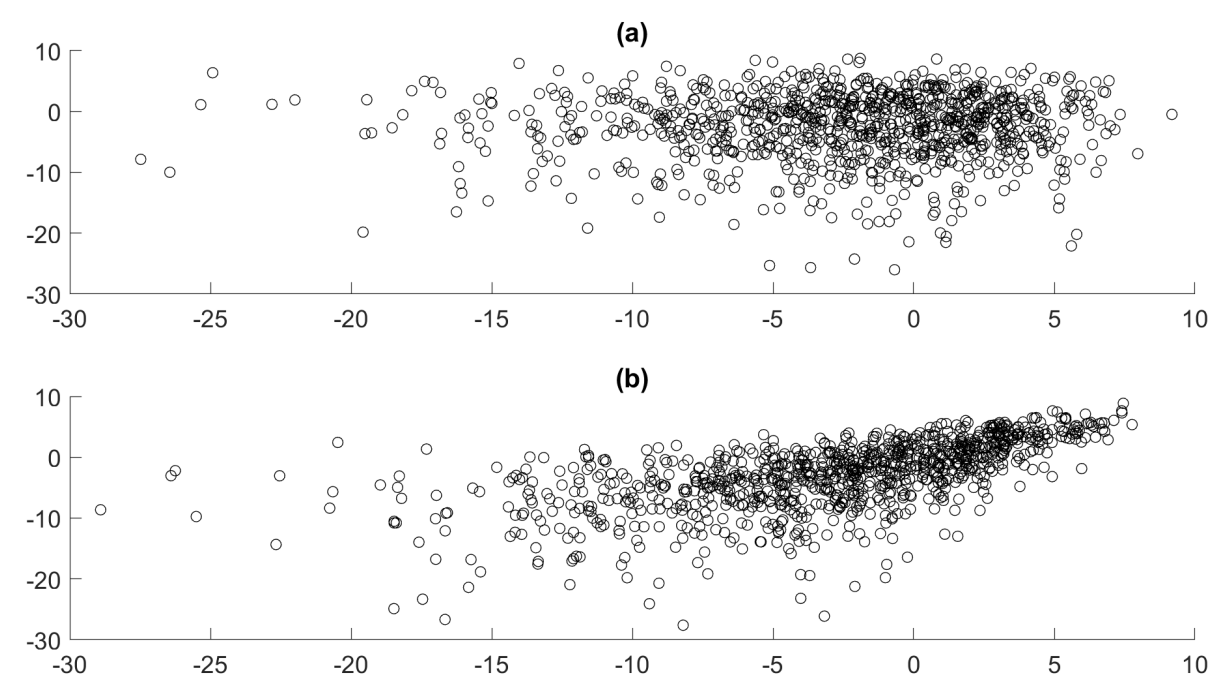

Figure 5.7: Scatter plots shows (a) comparison of two uncorrelated Rayleigh envelope sample series, (b) a comparison of two Rayleigh envelope sample series with $50 \%$ correlation

\subsection{Simulation Results}

For a fair comparison, the transmit power $P_{t}$ is assumed to be the same in all frequency bands. It should be noted that in a D-MIMO case the SNR at the receiver changes for each channel realization instead being fixed; therefore, the channel characteristics affect both the SNR and the MIMO matrix in the capacity equation. Also, as in the 
experiments, the Tx and $\mathrm{Rx}$ antennas are assumed to be omnidirectional at $2 \mathrm{GHz}$ with -2 dB gain, whereas, directional antennas are assumed at the upper bands with $11 \mathrm{~dB}$ and $16 \mathrm{~dB}$ gain for the Tx and the Rx, respectively. These values are considered achievable using steerable antenna arrays. The bandwidth is assumed to be $20 \mathrm{MHz}$ as in a typical OFDM system, and the noise figure of the receiver is assumed to be $9 \mathrm{~dB}$, which is typical of current user equipment. The same bandwidth was assumed for all frequency bands to allow the investigation of capacity differences that results from propagation characteristics even though it is realized that in ultimate systems wider bandwidth would be used at higher frequencies. At $18 \mathrm{GHz}$ and $28 \mathrm{GHz}$, the antenna at each DAU is assumed to be a sector antenna with the same beamwidth as the horns used in the experiments and with similar down tilt. At $2 \mathrm{GHz}$ the antenna at each DAU is assumed to be omnidirectional and at desktop height as that in the experiments.

The two main goals of this simulation were:

1. To compare the performance of systems operating at $18 \mathrm{GHz}$ and $28 \mathrm{GHz}$ in a cluttered indoor environment using beamforming at the UE toward a direction where the best signal strength is received with a system operating at $2 \mathrm{GHz}$ in the same environment while using omnidirectional antennas. Such a comparison is believed to provide a realistic picture whether systems operating at $18 \mathrm{GHz}$ and $28 \mathrm{GHz}$ can provide multi-user capacities comparable with that currently typical at $2 \mathrm{GHz}$ with currently available technology (i.e. steerable arrays with $16 \mathrm{~dB}$ gain and a transmit power of $10 \mathrm{dBm}$ ).

2. To compare the system performance of different antenna deployments in the upper bands. Such a comparison will give us insight into accepting or rejecting 
the initial claim that distributed multi-antenna systems can mitigate signal attenuation and system capacity dramatically.

\subsubsection{Results for $18 \mathrm{GHz}$ band}

Figure 5.9 shows cumulative probability distribution functions (CDFs) for capacity at $18 \mathrm{GHz}$, for a case where spatial variation is considered to have a Ricean distribution with ( $\mathrm{K}=7 \mathrm{~dB}$ ), and the temporal fading is assumed to be bursty Ricean ( $\mathrm{K}=7$ $\mathrm{dB})$. The D-MIMO links were modelled using the $L_{U G A}$ and $I P L V$ that were obtained from measurements. bursty fading is simulated as discussed in Appendix B.

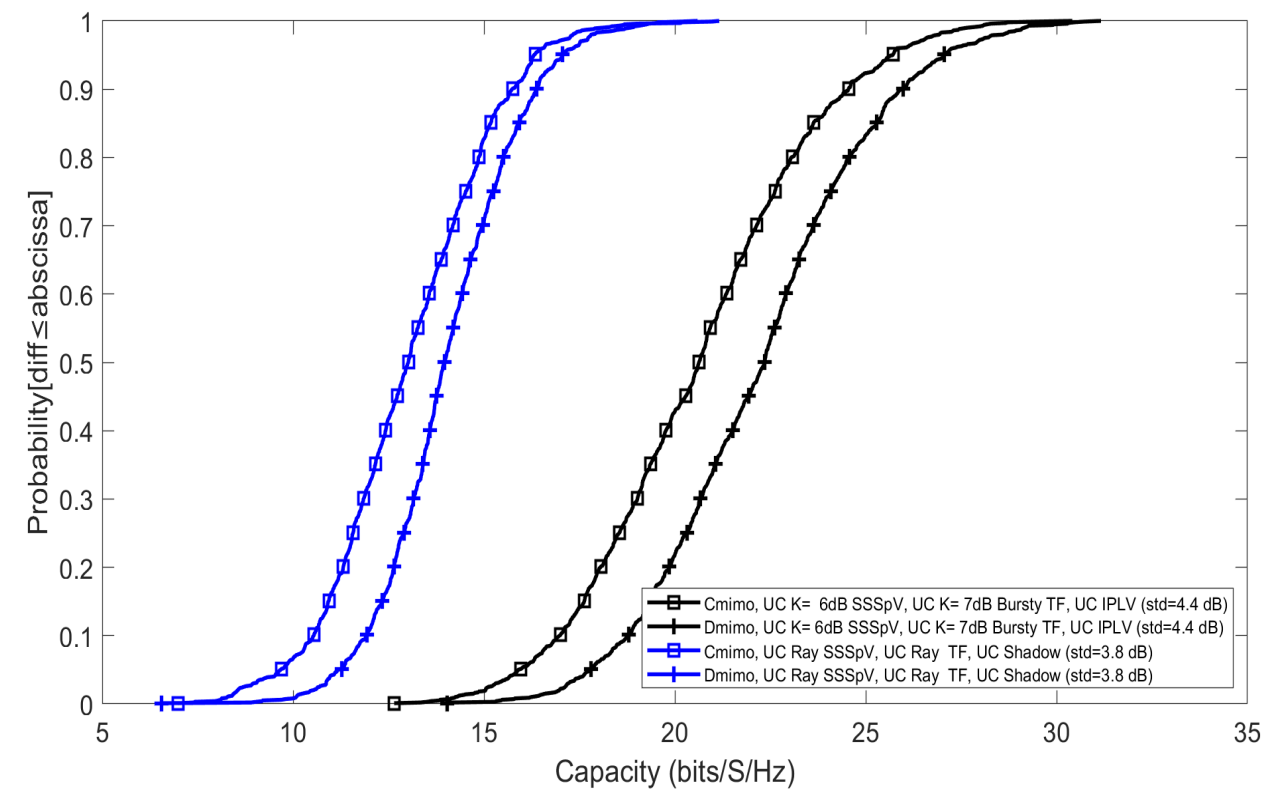

Figure 5.8: CDFs for system capacity for IPC in the $18 \mathrm{GHz}$ (Black), and omni 2 $\mathrm{GHz}$ (Blue)

Rayleigh SSSpVs and continuous Rayleigh temporal fading are assumed at $2 \mathrm{GHz}$, 
which is considered to be typical in indoor environment when omnidirectional antennas are used. As can be seen, by using elevated sector DAU antennas and narrow beam UE antennas with the ability to steer toward locations of maximum received powers at $18 \mathrm{GHz}$, a system gain of almost $6 \mathrm{bits} / \mathrm{s} / \mathrm{Hz}$ outage capacity at $1 \%$ can be achieved over that achievable with currently typical system operating at $2 \mathrm{GHz}$ with omnidirectional antennas. The average SNR for the $18 \mathrm{GHz}$ C-MIMO ${ }^{3}$ case is 19.5 $\mathrm{dB}$ which is $2.5 \mathrm{~dB}$ less than that for $18 \mathrm{GHz}$ D-MIMO case. The improvement of SNRs in D-MIMO scenarios is due to the advantages of reducing the access distance on average and acquiring micro diversity.

Correlation in both shadowing and fading has a significant impact on the system capacity as it decreases the $\mathbf{H}$ matrix rank and the capacity decreases as a result. It is anticipated that D-MIMO coupled with beamforming at upper bands will be able to decrease correlation between links greatly; however, this phenomenon has not been checked during measurements campaign, and therefore it is essential to consider all possibilities in simulation.

Figure 5.10 shows capacity results for the same system scenario associated with the results shown in Figure 5.9 but after introducing $50 \%$ correlation in both shadowing/IPLV and fading. As expected when introducing a correlation effect, a loss of capacity is observed. This increases when correlation coefficient is increased. Given the eigenvalues of the channel correlation matrix $\mathbf{H H}^{+}$, The effective degree of freedom dropped from 3.96 to 3.32 when correlation is introduced in the $18 \mathrm{GHz}$ case. By comparing the effect of correlation at both frequency bands, it can be seen that correlation can have more impact on upper bands, i.e. at $1 \%$ outage the capacity dropped

\footnotetext{
${ }^{3}$ In the C-MIMO deployment, all DAUs are assumed to be grouped in one location at the end of the room, above ceiling at $18 \mathrm{Ghz}$ and $28 \mathrm{GHz}$, and at desktop height at $2 \mathrm{GHz}$ )
} 
2 bits/s/Hz at $2 \mathrm{GHz}$ but dropped $3 \mathrm{bits} / \mathrm{s} / \mathrm{Hz}$ at $18 \mathrm{GHz}$ when same correlation was introduced.

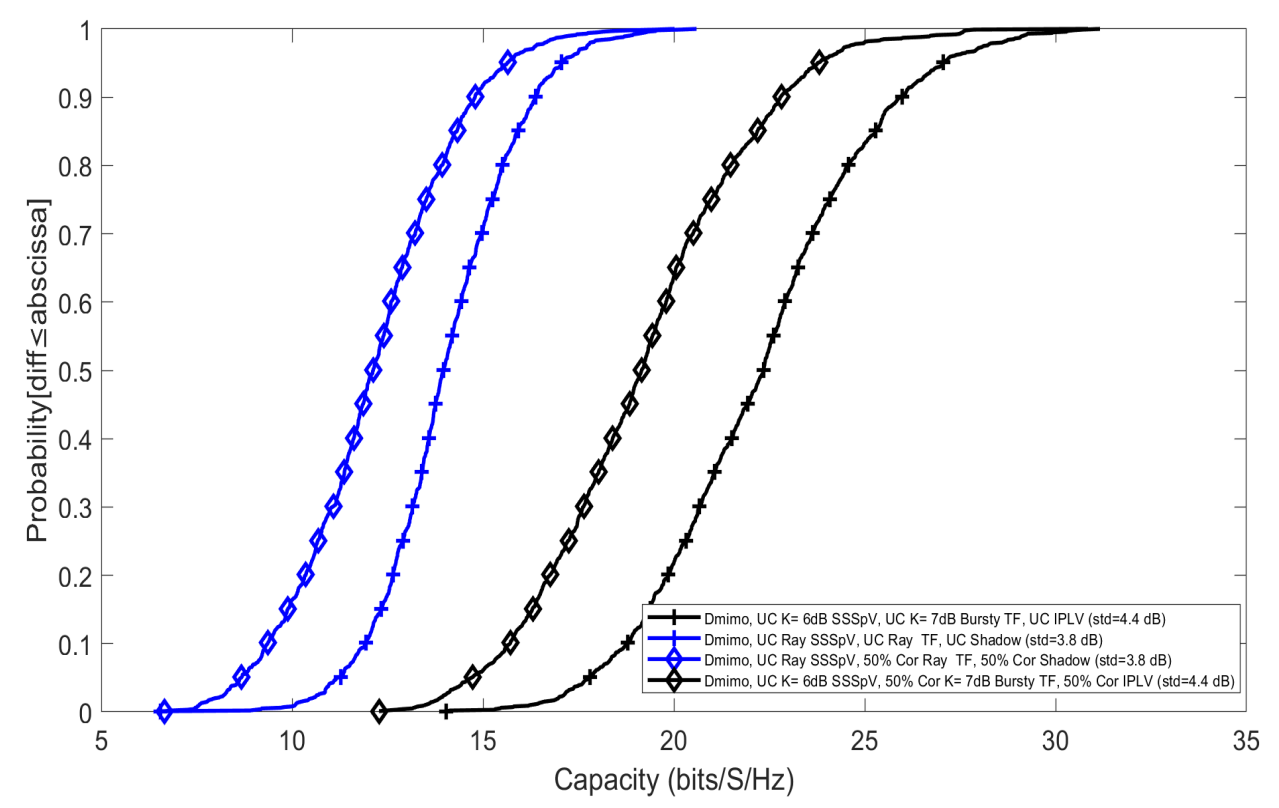

Figure 5.9: CDFs for system capacity for IPC in the $18 \mathrm{GHz}$ (Black), and omni 2 $\mathrm{GHz}$ (Blue) with $50 \%$ correlation in both shadowing and fading

\subsubsection{Results for $28 \mathrm{GHz}$ band}

Similar to the discussion in the foregoing section, this section discusses the system capacity of C-MIMO and D-MIMO at $28 \mathrm{GHz}$. The results of $2 \mathrm{GHz}$ presented in the previous section are also used here as a reference. As opposed to in the $18 \mathrm{GHz}$ case, Figure 5.11 shows a decrease in system capacity with respect to that achievable at 2 $\mathrm{GHz}$ at $28 \mathrm{GHz}$ under the same channel conditions as those simulated for $18 \mathrm{GHz}$. It is considered that this decreasing capacity is probably a result of the increase in the propagation and obstruction loss at this higher band. The system capacity decreases $3.5 \mathrm{bits} / \mathrm{s} / \mathrm{Hz}$ when compared with the D-MIMO case at $2 \mathrm{GHz}$. To achieve the same 
outage capacity at $1 \%$ level as a system operating at $2 \mathrm{GHz}$, an increase in total transmit power of $16 \mathrm{dBm}$ is required as can be seen from the the curve marked with triangles, where $4 \mathrm{dBm}$ transmit power increase was applied at each of the 4 transmit antennas.

Also, by comparing the C-MIMO and D-MIMO cases at $28 \mathrm{GHz}$, it can be observed that an increase of system capacity of almost $1.5 \mathrm{bits} / \mathrm{s} / \mathrm{Hz}$ can be achieved bu using D-MIMO instead of C-MIMO, and this can reduce the required total transmit power.

In passing, the reader should note from the foregoing figures that spatial multiplexing can still be achieved with Ricean fading even when K-factor is high. This is considered important since fading channels at higher bands for systems with narrow beams antennas are Ricean and not Rayleigh which is often considered required for a MIMO matrix to have a full rank, so the MIMO gain can be achieved.

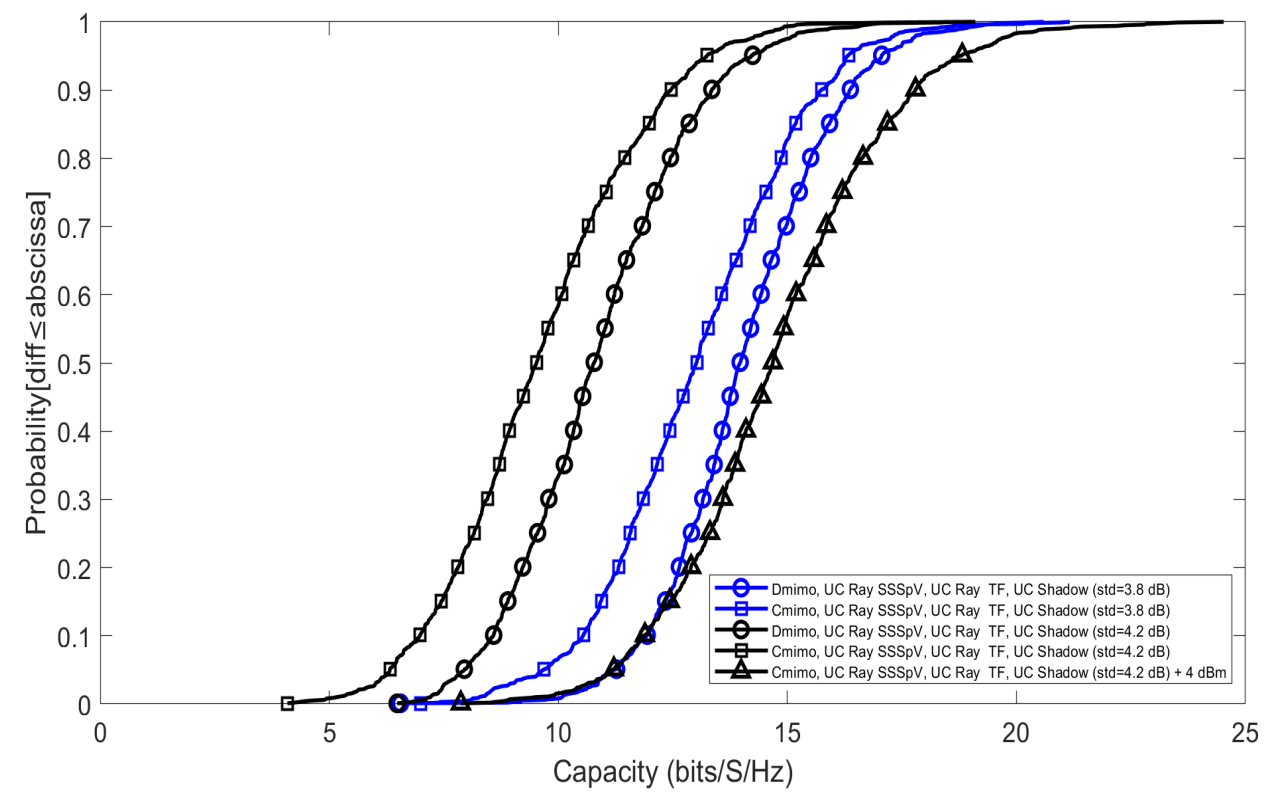

Figure 5.10: CDFs for system capacity for IPC in the $28 \mathrm{GHz}$ (Black), and omni 2 $\mathrm{GHz}$ (Blue) 
In addition to the correlation effects on system capacity, Figure 5.12 shows also the impact of interference on system capacity at $28 \mathrm{GHz}$. The importance of presenting these two calculations together is the fact that at $2 \mathrm{GHz}$ when there is no beamforming, there might be no way to avoid correlation in shadowing, temporal fading, and interference. However, avoiding correlation and interference when narrow beam steering could be feasible if appropriate planing of antenna placement is considered.

Interference was simulated using the inverse distance law after considering an extra obstruction loss caused by walls of the room since the interfere was assumed to be in one of the neighbouring rooms. The interfering transmitter was assumed to have the same antenna as those at DAUs. The interfering signal was assumed to undergo bursty Ricean fading and Ricean SSSpVs.

Figure 5.12 also shows the CDF of D-MIMO system capacity in the $28 \mathrm{GHz}$ band when correlated Ricean fading channels and correlated IPLVs are present (Red with diamond markers). Also shown is the CDF of system capacity when interference is present (red with x markers). As expected, correlation causes the capacity to decrease, and further loss of the capacity caused by the interference. It is observed that a loss of $3 \mathrm{bits} / \mathrm{s} / \mathrm{Hz}$ can be saved if correlation and interference are avoided. 


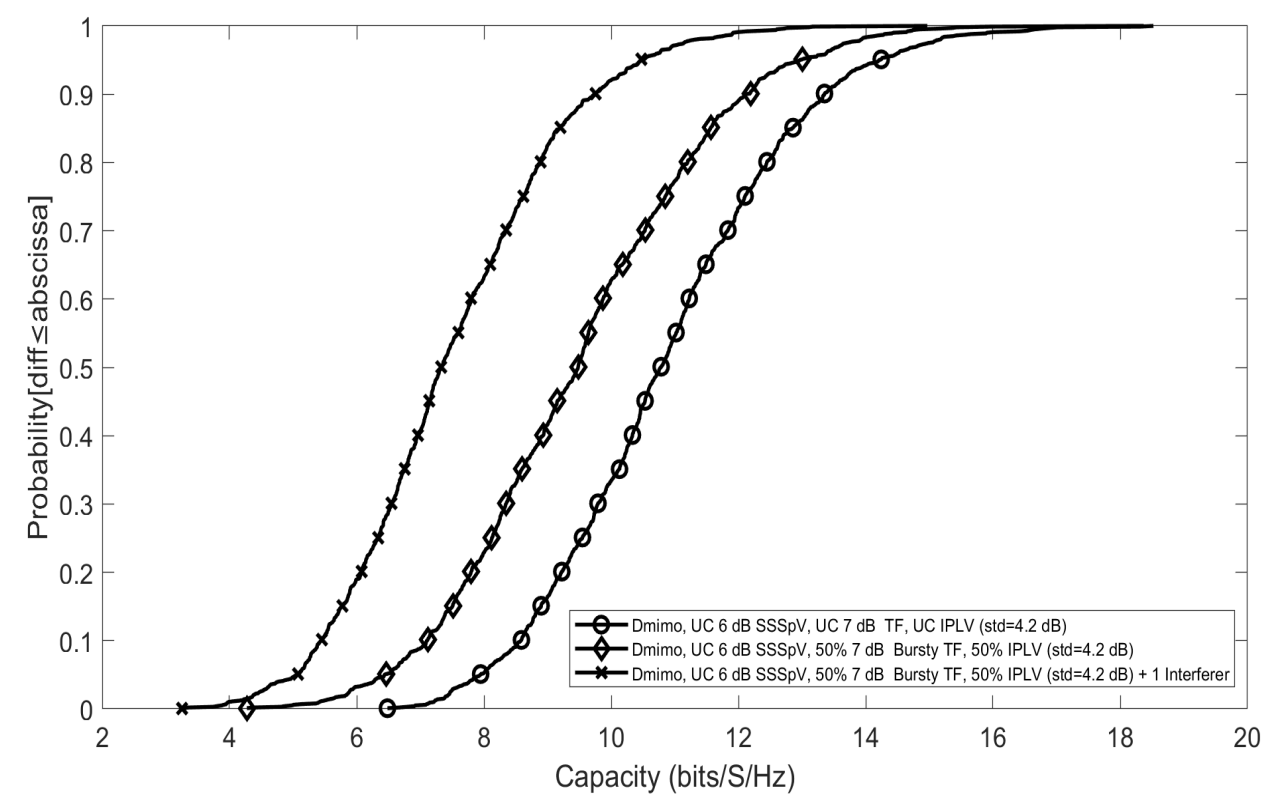

Figure 5.11: CDFs for system capacity for IPC in the $28 \mathrm{GHz}$

In the interest of explaining the foregoing results, the similarity of eigenvalues, referred to $G_{m u x}$ in the literature [40], was calculated using the equation

$$
G_{m u x}=\frac{\left(\prod_{k=1}^{K} \lambda_{k}\right)^{\frac{1}{K}}}{\frac{1}{K} \sum_{k=1}^{K} \lambda_{k}},
$$

which is the ratio of geometric and arithmetic means of the eigenvalues of $\mathbf{H H}^{+}$. This is used as a measure for relative differences between the powers of eigenvalues - $G_{m u x}$ is a function of all eigenvalues, which makes it a useful figure of merit to characterize the spread of eigenvalues by using a single number. Figure 5.13 shows three CDFs for $G_{\text {mux }}$ for D-MIMO scenario at $28 \mathrm{GHz}$ for cases where no correlation, $50 \%$ correlation, and $80 \%$ correlation in both IPVL and temporal fading. As can be seen from the figure when correlation is introduced the $G_{m u x}$ decreases, and it is the 
least for $80 \%$ correlation in which the eigenvalues were always lower.

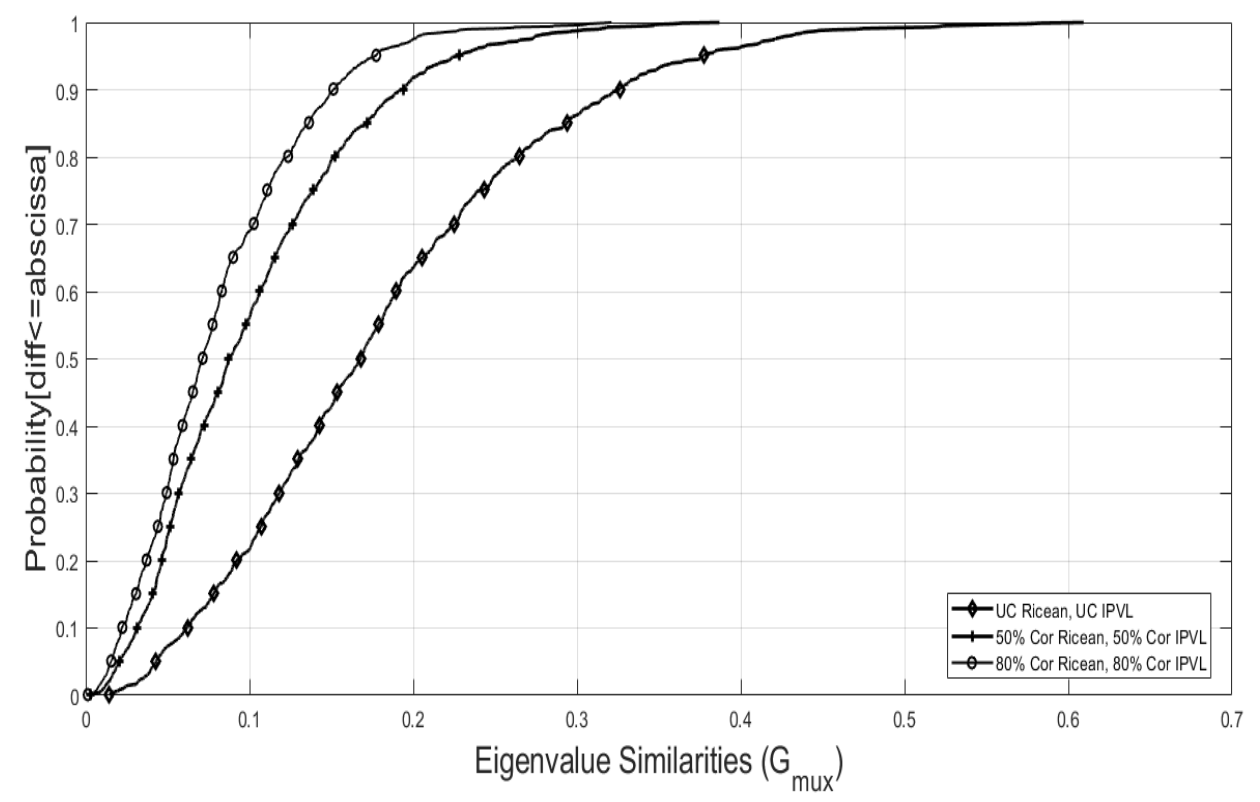

Figure 5.12: ECDFs for $G_{m u x}$, the ratio of geometric and arithmetic means of the eigenvalues

A comparison of outage capacity for which $P\left[C>C_{\text {out }}\right]=0.99$ at $18 \mathrm{GHz}$ for different scenarios is presented in Figure 5.14. For the associated simulations the transmit power was effectively varied so the SNR was the same in all locations, even for D-MIMO. It is shown that the maximum outage capacities are achieved when there are no correlations in the IPLVs nor in the small scale fading of a D-MIMO system, it is also slightly higher than the case of a C-MIMO with uncorrelated Rayleigh fading which is considered the best scenario to achieve a spatial multiplexing in conventional MIMO. The minimum values of outage capacity result when there are correlated Rayleigh fading and correlated IPLVs. The fact that beam-steering can be achieved with small antenna arrays at $18 \mathrm{GHz}$ and $28 \mathrm{GHz}$ might allow the avoidance of 
correlation in a DAS at these frequencies.

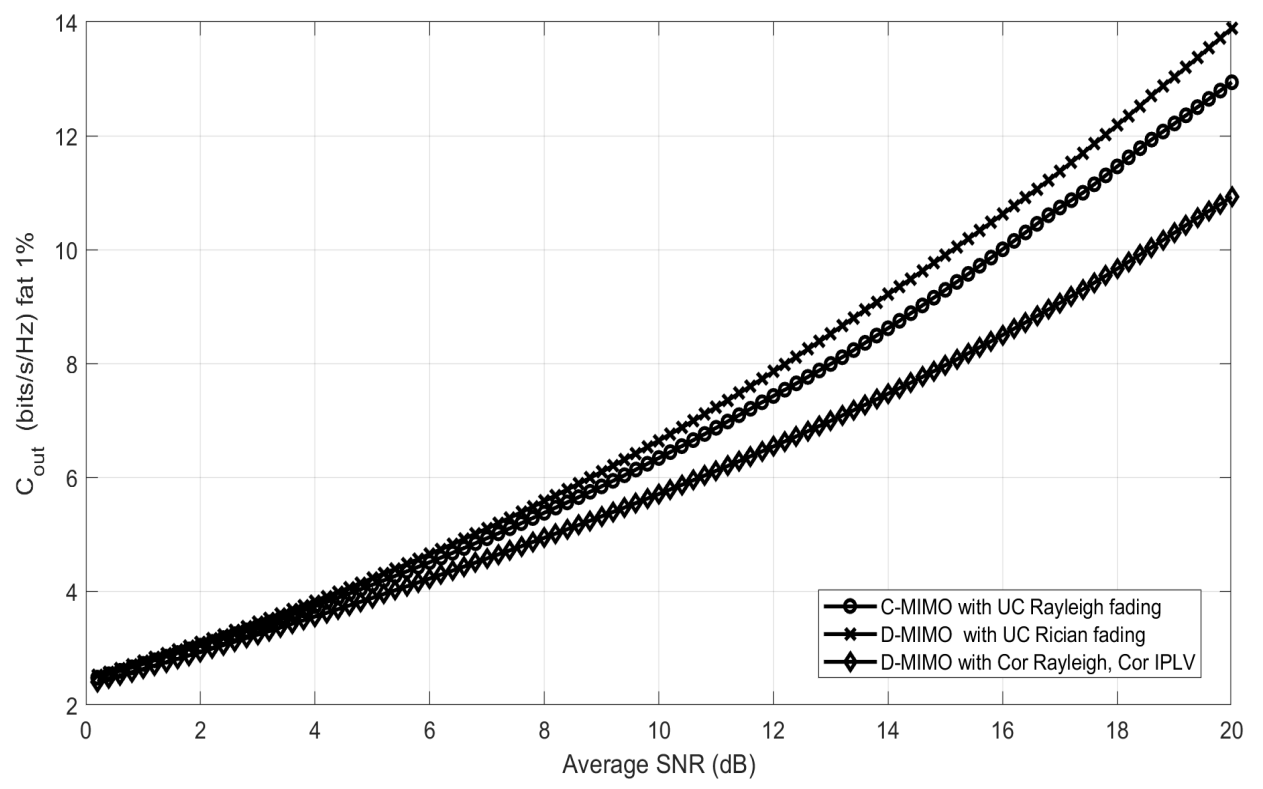

Figure 5.13: Outage capacity as a function of average SNR for IPC in the $18 \mathrm{GHz}$ band for 3 different scenarios 


\section{Chapter 6}

\section{Summary and Conclusion}

With the evolution of 4 th generation $4 \mathrm{G}$ wireless system to $5 \mathrm{G}$, access to wireless

spectrum that will drive this evolution becomes crucially important. Industry is therefore studying how to make available more wireless channels, which are expected to be allocated at frequencies above $6 \mathrm{GHz}$. However, a significant problem in the use of frequency bands above $6 \mathrm{GHz}$ in cluttered indoor environments is increased transmission and obstruction loss. Research was therefore needed to characterize and model propagation characteristics on indoor channels above $6 \mathrm{GHz}$. This thesis has addressed the requirement through the conduct of wideband measurements at centre frequencies of $2,5,10,15,18,22$, and $28 \mathrm{GHz}$ frequency bands in a typical indoor small office environment.

The research began with the conduct of phenomenological measurements in order to better understand wireless indoor channels at the above mentioned frequencies, and to study some phenomena that could enable indirect path communication at higher frequencies including a conjecture that more object in a cluttered indoor environment will become better reflector at higher frequencies and the fact that waves can pass through gaps between obstructions with lower loss. 
,These measurements were made in a small room with furniture layouts specifically designed to conduct these measurements. Then, after rearranging the furniture and adding more so as to achieve an environment that is typical of modern small offices, channel measurements were conducted at $18 \mathrm{GHz}$ and $28 \mathrm{GHz}$ in which transmission was from an emulated sector antenna mounted with a down-tilt of 62.5 degrees above the suspended ceiling in the room at the height of $4 \mathrm{~m}$ above floor level. Reception was at 45 desk-top-height locations at $18 \mathrm{GHz}$ and a subset of 22 of these locations at $28 \mathrm{GHz}$ using step-wise azimuth and elevation scanning horn antennas with $10 \mathrm{deg}$ beamwidth in both planes. To enable a comparison with results from a $2 \mathrm{GHz}$ system with a currently typical configuration, measurements were also made at $2 \mathrm{GHz}$ when transmission was from an omnidirectional antenna centered at the end of the room at desk height and reception was with a $2 \mathrm{GHz}$ monopole at the same 45 desktop height locations where measurements were made at $18 \mathrm{GHz}$. Transmission loss, shadowing, and indirect path loss variation models were derived from these measurements. Temporal fading characteristics were also studied and modelled. To mitigate the effect of multipath interference in transmission loss modelling, averaging over frequency was used in place of averaging over small local areas in space. This was conjectured to be possible based on heuristic argument and demonstrated to be effective in transmission loss vs distance measurements in an empty room at 6 of the centre frequencies used in the work reported herein. Using such averaging is considered to be a significant improvement with respect to transmission loss measurements and modelling conducted by others in which no steps are taken to mitigate multipath effects.

The main goal of this thesis was to study whether wireless systems operating at SHF with currently available technology can deliver the same system capacity as 
currently typical systems at $2 \mathrm{GHz}$. Results from propagation measurements showed that in the same environment by using directive antennas to take advantage of indirect path via a reflections of opportunity, average transmission loss was $25 \mathrm{~dB}$ higher at $18 \mathrm{GHz}$ and $35 \mathrm{~dB}$ higher at $28 \mathrm{GHz}$ than with non-directive conventional communication coverage configuration at $2 \mathrm{GHz}$. These values compare with $19 \mathrm{~dB}$ and 24 $\mathrm{dB}$, respectively, in free space. It was also found that when strong specular reflections are used in IPC, RMS delay spread are reduced to values in the range of 3 to 5 ns and become independent of frequency. Additionally, it was found that received powers within $3 \mathrm{~dB}$ of maximum can be found in multiple directions using beamsteering. This is considered important for redundancy in a mitigation of co-channel interference and the reduction of correlations in channel dynamics, which are well known to reduce system capacity. Finally, it was found that narrowband temporal variations on indirect paths that result from motion in an environment of operation are bursty, and during fading bursts, fading could be modelled as having a Ricean distribution with $\mathrm{K}$ factors ranging from 6 to $8 \mathrm{~dB}$.

Multi-user distributed antenna system capacity calculations for 4x4 D-MIMO channels were made based on channel simulations conducted using transmission loss, shadowing, indirect path loss variation, and temporal fading models derived from the measurements. Included was consideration of local area spatial variations in received power with respect to average transmission loss models which is not often included in other studies reported in literature. The results from these simulations show that:

1. With currently available technology and steerable narrow beam antennas at user devices that can be steered to take advantage of strong signals from reflectors of opportunity and gaps between obstructions to avoid excessive diffraction 
loss in clutter, narrowband systems can be designed at $18 \mathrm{GHz}$ and $28 \mathrm{GHz}$ to support the same multi-user capacity as narrowband systems with typical current configurations at $2 \mathrm{GHz}$. Further, it is anticipated that since greater bandwidth are likely to be allocated at the higher frequencies than at $2 \mathrm{GHz}$, even greater capacity can be achieved using wider operating bandwidths than at $2 \mathrm{GHz}$.

2. In a D-MIMO system, spatial multiplexing can still be achieved with Ricean fading even when $\mathrm{K}$ factors are high, which was shown to be the case in IPC system at $18 \mathrm{GHz}$ and $28 \mathrm{GHz}$.

3. As in conventional systems in IPC systems D-MIMO outperforms C-MIMO. D-MIMO yield greater system capacity than C-MIMO

4. It is conjectured that signals with powers withing $3 \mathrm{~dB}$ of the maximum can be obtained in multiple directions in IPC system, correlations in channel dynamics and co-channel interference can both be mitigated which would lead to even more capacity gains at higher frequencies where antenna arrays can be designed with compact sizes.

\subsection{Future Work}

In order to complement our study the following further work is suggested:

- The fact that near maximum power can be received from several different pointing directions led to the conjecture that beam steering can also be used to mitigate interference and correlation, however, this need to be further investigated. 
- Simulations reported in this thesis were constrained to be conducted assuming the same beamwidths as those use for the propagation experiments, additional work can be conducted using measurements equipment that allows super resolution analysis of measured data to eliminate this constraint followed with simulation with other UE antenna beamwidth of interest.

- Similar measurements and modelling for typical larger open office environment need to be conducted.

- Capacities for systems with wider bandwidth at higher frequencies should be calculated.

- Work should be done to find an analytical relationship between local area averages of transmission loss centred on a specific Tx-Rx antenna separation and averages across frequency of powers in spectral lines of broadband signals received at the same Tx-Rx antenna separation. 


\section{Bibliography}

[1] M. Cudak et al. Moving towards mmWave-based beyond-4G (B-4G) technology. 2013 IEEE 7rth Vehicular Technology Conference (VTC Spring), Dresden, pages 1-5, 2013.

[2] T. S. Rappaport et al. Millimeter wave mobile communications for 5G cellular: It will work! IEEE Access, 1:335-349, 2013.

[3] Richard N Clarke. Expanding mobile wireless capacity: The challenges presented by technology and economics. 19th ITS Biennial Conference, Bangkok, 2012.

[4] Juan Yang Qian Chen, Xiaoming Peng and F. Chin. Spatial reuse strategy in mmWave WPANs with directional antennas. 2012 IEEE Global Communications Conference (GLOBECOM), Anaheim), 1:5392-5397, 2012.

[5] N. Seifi, A. Wolfgang, and T. Ottosson. Downlink performance and capacity of distributed antenna systems based on realistic channel model. 2008 International ITG Workshop on Smart Antennas, pages 249-253, 2008.

[6] S. Baek, Y. Chang, H. Kim, and A. Agiwal. Comparison analysis of outdoor channel characteristics at $28 \mathrm{GHz}$ and $2 \mathrm{GHz}$ using 3D Ray-Tracing technique. 
2014 IEEE 80th Vehicular Technology Conference (VTC2014-Fall), pages 1-5, Sep. 2014.

[7] G. R. MacCartney and T. S. Rappaport. $73 \mathrm{GHz}$ millimeter wave propagation measurements for outdoor urban mobile and backhaul communications in New York city. 2014 IEEE International Conference on Communications (ICC), pages 4862-4867, June 2014.

[8] T. S. Rappaport, E. Ben-Dor, J. N. Murdock, and Y. Qiao. $38 \mathrm{GHz}$ and $60 \mathrm{GHz}$ angle-dependent propagation for cellular peer-to-peer wireless communications. 2012 IEEE International Conference on Communications (ICC), pages 45684573, June 2012.

[9] G. R. Maccartney, T. S. Rappaport, S. Sun, and S. Deng. Indoor office wideband millimeter-wave propagation measurements and channel models at 28 and 73 GHz for ultra-dense 5G wireless networks. IEEE Access, 3:2388-2424, 2015.

[10] S. Sun, H. Yan, G. R. MacCartney, and T. S. Rappaport. Millimeter wave smallscale spatial statistics in an urban microcell scenario. 2017 IEEE International Conference on Communications (ICC), pages 1-7, May 2017.

[11] H. Hashemi, M. McGuire, T. Vlasschaert, and D. Tholl. Measurements and modeling of temporal variations of the indoor radio propagation channel. IEEE Transactions on Vehicular Technology, 43(3):733-737, Aug 1994.

[12] P. Marinier, G. Y. Delisle, and C. L. Despins. Temporal variations of the wideband indoor wireless millimeter-wave channel. IEEE Antennas and Propagation Society International Symposium 1997. Digest, 2:592-597 vol.2, July 1997. 
[13] Ou Zhao, Hidekazu Murata, and Susumu Yoshida. Channel capacity of distributed MIMO antenna systems under the effect of spatially correlated shadowing. Vehicular Technology Conference, 1988, IEEE 38th, pages 1-5, 092013.

[14] Wonil Roh and A. Paulraj. Outage performance of the distributed antenna systems in a composite fading channel. Proceedings IEEE 56th Vehicular Technology Conference, 3:1520-1524 vol.3, Sep. 2002.

[15] R. Bultitude. Measurement, characterization and modeling of indoor 800/900 $\mathrm{MHz}$ radio channels for digital communications. IEEE Communications Magazine, 25(6):5-12, 1987.

[16] T. S. Rappaport. Characterization of UHF multipath radio channels in factory buildings. IEEE Trans. Antennas Propag., 37(8):1058-1069, 1989.

[17] C. R. Anderson and T. S. Rappaport. In-building wideband partition loss measurements at 2.5 and $60 \mathrm{GHz}$. IEEE Trans. Wireless Commun., 3(3):922-928, 2004.

[18] C. R. Anderson et al. In-building wideband multipath characteristics at 2.5 and 60 ghz. Proc. IEEE 56th Veh. Technol. Conf. (2002 IEEE VTC-Fall), 1:97-101, 2002.

[19] T. J. Beukema T. Zwick and Haewoon Nam. Wideband channel sounder with measurements and model for the $60 \mathrm{GHz}$ indoor radio channel. IEEE Transactions on Vehicular Technology, 54:1266-1277, 2005.

[20] J. Kivinen S. Geng and P. Vainikainen. Propagation characterization of wideband indoor radio channels at $60 \mathrm{GHz}$. 2005 IEEE International Symposium on 
Microwave, Antenna, Propagation and EMC Technologies for Wireless Communications, Beijing, 1:314-317, 2005.

[21] T. Lei M. Lei, J. Zhang and D. Du. 8-GHz indoor channel measurements and analysis of propagation characteristics. 2014 IEEE 25th Annual International Symposium on Personal, Indoor, and Mobile Radio Communication (PIMRC), Washington, DC, pages 208-212, 2014.

[22] S. Deng G. R. MacCartney and T. S. Rappaport. Indoor office plan environment and layout-based mmWave path loss models for $28 \mathrm{GHz}$ and $73 \mathrm{GHz}$. 2016 IEEE 83rd Vehicular Technology Conference (VTC Spring), Nanjing, pages 1-6, 2016.

[23] S. Sun M. K. Samimi, G. R. MacCartney and T. S. Rappaport. $28 \mathrm{GHz}$ millimeter-wave ultrawideband small-scale fading models in wireless channels. 2016 IEEE 83rd Vehicular Technology Conference (VTC Spring), Nanjing, 6:16, 2016.

[24] A. Rustako A. A. M. Saleh and R. Roman. Distributed antennas for indoor radio communications. IEEE Transactions on Communications, 35(12):1245$1251,1987$.

[25] P. Padilla Juan F. Valenzuela-Valds Santiago Gonzlez-Aurioles, J. L. Padilla and Juan C. Gonzalez-Macias. On the MIMO capacity for distributed system under composite Rayleigh/Rician fading and shadowing. International Journal of Antennas and Propagation, 2015(12), 2015. 
[26] Zhang Fenghua Tian Lei Sheng Nan, Zhang Jianhua. Downlink performance of indoor distributed antenna systems based on wideband MIMO measurement at 5.25 GHz. IEEE Vehicular Technology Conference, pages 1-5, 2011.

[27] S. Salous. Radio propagation measurement and channel modelling. John Wiley and Sons Ltd, 2013.

[28] Theodore S Rappaport. Wireless communications: Principles and practice, 2nd edition. Prentice Hall, 2002.

[29] T A Wilkinson J T E Mcdonnell, T P Spiller. RMS delay spread in indoor LOS environments at 5.2 GHz. HP Laboratories Bristol, HPL-98-63, 1998.

[30] M. K. Samimi S. Deng and T. S. Rappaport. $28 \mathrm{GHz}$ and $73 \mathrm{GHz}$ millimeterwave indoor propagation measurements and path loss models. 2015 IEEE International Conference on Communication Workshop (ICCW), London, pages $1244-1250,2015$.

[31] M. A. Stephens. EDF statistics for goodness of fit and some comparisons. Journal of the American Statistical Association, page 730737, 1974.

[32] B. Liu, K. Lin, and J. Chen. Ricean K-factor estimation in cellular communications using Kolmogorov-Smirnov statistic. 2006 Asia-Pacific Conference on Communications, pages 1-5, Aug 2006.

[33] Larry Greenstein, D.G. Michelson, and V. Erceg. Moment-method estimation of the Ricean K-factor. Communications Letters, IEEE, 3:175 - 176, 071999. 
[34] Liang Xiao, Lin Dai, Hairuo Zhuang, Shidong Zhou, and Yan Yao. Informationtheoretic capacity analysis in MIMO distributed antenna systems. IEEE Vehicular Technology Conference, 1:779 - 782 vol.1, 052003.

[35] Huaiyu Dai. Distributed versus co-located MIMO systems with correlated fading and shadowing. 2006 IEEE International Conference on Acoustics Speech and Signal Processing Proceedings, 4:IV-IV, May 2006.

[36] Huaiyu Dai, Hongyuan Zhang, and Zhou Quan. Some analysis in distributed MIMO systems. Journal of Communications, 2, 052007.

[37] Shu Sun, Hangsong Yan, George Maccartney, and T.S. Rappaport. Millimeter wave small-scale spatial statistics in an urban microcell scenario. 032017.

[38] Yang Wen Liang. Ergodic and outage capacity of narrowband MIMO Gaussian channels. 012005.

[39] A. Goldsmith, S. A. Jafar, N. Jindal, and S. Vishwanath. Capacity limits of MIMO channels. IEEE Journal on Selected Areas in Communications, 21(5):684702, June 2003.

[40] L. Vuokko J. Kivinen K. Sulonen P. Suvikunnas, J. Salo and P. Vainikainen. Comparison of MIMO antenna configurations: Methods and experimental results. IEEE Transactions on Vehicular Technology, 57:1021-1031, 2008.

[41] C. A. Gutierrez Diaz de Leon, M. C. Bean, and J. S. Garcia. On the generation of correlated Rayleigh envelopes for representing the variant behavior of the indoor radio propagation channel. 2004 IEEE 15th International Symposium on 
Personal, Indoor and Mobile Radio Communications (IEEE Cat. No.04TH8754), 4:2757-2761 Vol.4, Sep. 2004. 


\section{Appendix A}

\section{Rayleigh and Ricean Distribution Functions}

\section{RAYLEIGH FADING}

Rayleigh fading is a reasonable model when there are many objects in the environment that reflect and scatter the radio signal before it arrives at the receiver. The central limit theorem holds that, if there are sufficiently many scattered and reflected so-called multipath signal components, the receive signal can be well-modelled as a Gaussian process. If there is no coherent signal component, then such a process will have zero mean and a phase uniformly distributed between 0 and $2 \pi$ radians. The envelope of the receive signal will therefore be Rayleigh distributed.

$$
F_{R}(r)=1-e^{\frac{r^{2}}{2 \sigma^{2}}}
$$

where $R$ is the envelope of the received signal and $\sigma^{2}$ is the received power and can be evaluated from the mean square value $\mathrm{R}$.

$$
E\left(r^{2}\right)=2 \sigma^{2}
$$

Often, the gain and phase of a channel are conveniently represented as a complex 
number. In this case, the real and imaginary parts of a receive signal that exhibit Rayleigh fading can be modelled as independent and identically distributed zero-mean Gaussian processes.

\section{RICEAN FADING}

Rician fading occurs when one of the receive signals, typically a line-of-sight signal or a specular reflection is coherent rather than random like the other multipath components. In Rician fading, the receive signal envelope is characterized by a Rician distribution. The cumulative distribution function of Ricean channel is given by

$$
F_{R}(r)=1-e^{-\frac{s^{2}+r^{2}}{2 \sigma^{2}}} \sum_{m=0}^{\infty}\left(\frac{s}{r}\right)^{m} I_{m}\left(\frac{r_{s}}{\sigma^{2}}\right)
$$

where $s$ and $\sigma^{2}$ are the parameters of Ricean distribution function and $I_{m}$ is the modified Bessel function of the first kind and $m^{\text {th }}$ order. If $R$ is Ricean random variable then $R^{2}$ is a non-central Chi-square random variable. Hence the parameters of a Ricean random variable can be evaluated from the moments of $R^{2}$

$$
\begin{gathered}
\mu=E\left(R^{2}\right)=2 \sigma^{2}+s^{2} \\
v=E\left[\left(R^{2}-\mu\right)^{2}\right]=4 \sigma^{4}+4 \sigma^{2} s^{2}
\end{gathered}
$$

The parameter of Ricean are usually defined in terms of the $K$ ratio, i.e.

$$
K=\frac{s^{2}}{2 \sigma^{2}}
$$




\section{Appendix B}

\section{Method of Generating Rayleigh and Ricean Fading Envelopes}

To study the impact of fading on the D-MIMO system performance, a procedure for generating Rayleigh and Ricean fading envelopes with any desired covariance matrix was required [41]. To generate $N$ Rayleigh envelops $\left(r_{1}, r_{2}, r_{3}, \ldots, r_{N}\right)$, first, the desired covariance matrix of the Rayleigh envelops is chosen as follow

$$
\hat{K}_{r}=\left[\begin{array}{ccccc}
\sigma_{r 1}^{2} & \hat{\rho}_{r_{1,2}} & \hat{\rho}_{r_{1,3}} & \ldots & \hat{\rho}_{r_{1, N}} \\
\hat{\rho}_{r_{2,1}} & \sigma_{r 2}^{2} & \hat{\rho}_{r_{2,3}} & \ldots & \hat{\rho}_{r_{2, N}} \\
: & & & & \\
\hat{\rho}_{r_{N, 1}} & \hat{\rho}_{r_{N, 2}} & \hat{\rho}_{r_{N, 3}} & \ldots & \sigma_{r N}^{2}
\end{array}\right]
$$

then the normalized covariance matrix is derived

$$
K_{r}=\left[\begin{array}{ccccc}
1 & \rho_{r_{1,2}} & \rho_{r_{1,3}} & \cdots & \rho_{r_{1, N}} \\
\rho_{r_{2,1}} & 1 & \rho_{r_{2,3}} & \cdots & \rho_{r_{2, N}} \\
: & & & & \\
\rho_{r_{N, 1}} & \rho_{r_{N, 2}} & \rho_{r_{N}, 3} & \cdots & 1
\end{array}\right]
$$


where $\rho_{r_{i, j}}=\hat{\rho}_{r_{i, j}} / \sqrt{\sigma_{r_{i}}^{2} \sigma_{r j}^{2}}$ and then for each cross correlation coefficient $\rho_{r_{i, j}}$ the cross correlation coefficients of the corresponding complex Gaussian random variables $\rho_{g_{i, j}}$ is computed using a linear interpolation as shown in Figure B.1. The resulted complex Gaussian covariance matrix becomes

$$
K_{g}=\left[\begin{array}{ccccc}
1 & \rho_{g_{1,2}} & \rho_{g_{1,3}} & \ldots & \rho_{g_{1, N}} \\
\rho_{g_{2,1}} & 1 & \rho_{g_{2,3}} & \ldots & \rho_{g_{2, N}} \\
: & & & & \\
\rho_{g_{N, 1}} & \rho_{g_{N, 2}} & \rho_{g_{N, 3}} & \ldots & 1
\end{array}\right]
$$

The next step is to generate $\mathrm{N}$ uncorrelated complex Gaussian samples $V=$ $v_{1}, v_{2}, v_{3}, \ldots, v_{N}$ each with variance $\sigma_{g}^{2}$ then determine the coloring matrix $L$ corresponding to $K_{g}$ such that $L L^{T}=K_{g}$ where $L^{T}$ represents the transpose of $L$ and generate correlated complex Gaussian samples using $W=L V$. The resulting $N$ envelopes of the Gaussian samples in $W$ correspond to Rayleigh random variables $\left(r_{1}^{\prime}, r_{2}^{\prime}, \ldots, r_{N}^{\prime}\right)$ with normalized covariance matrix $K_{r}$ and equal variance

$$
\sigma_{r}^{2}=\left(2-\frac{\pi}{2}\right) \frac{1}{2} \sigma_{g}^{2}
$$

Next, the desired Rayleigh envelopes $\left(r_{1}, r_{2}, \ldots, r_{N}\right)$ is created from the samples $\left(r_{1}^{\prime}, r_{2}^{\prime}, \ldots, r_{N}^{\prime}\right)$ by evaluating $r_{i}=A_{i} \cdot r_{i}^{\prime}$ where $A_{i}=\sigma_{r i} / \sigma_{r}$. In order to generate $N$ Ricean envelopes, we follow the same procedure, but with adding a constant $C$ to one of the zero mean Gaussian random variables in Eq (5.4) $r_{i}=\sqrt{\left(C+x_{i}\right)^{2}+y_{i}^{2}}$ where $C^{2}+2 \sigma_{i}^{2}=1$ and $K=\frac{C^{2}}{2 \sigma_{i}^{2}}$.

The event of temporal fading on fixed indoor links depend on the link-antenna radiation patterns. In the case of antennas with narrow beamwidths, like those used 


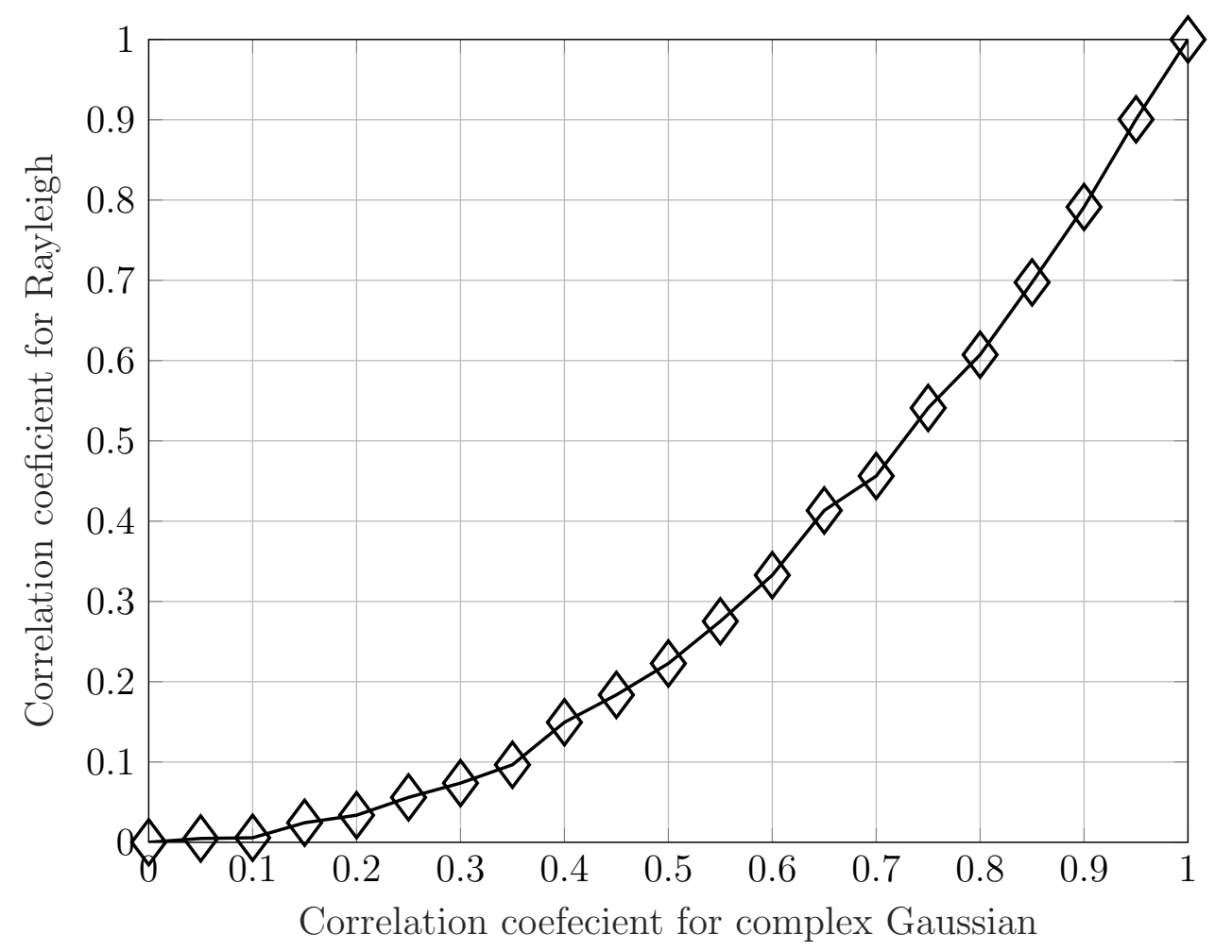

Figure B.1: Relationship between correlation coefficients of complex Gaussian $\rho_{g_{i j}}$ and Rayleigh $\rho_{r_{i j}}$

in the experiments, the fading cannot be continuous unless the motion always occurs in an area illuminated by the antenna beamwidth. However in a realistic scenario, the fading is is discrete in which a burst of fade occurs from time to time. In that case, fading interval starts with a shallow fluctuation in the received power, then a deep fade occurs when an obstructing person provides partial or total blockage at boresight, followed by another fast fluctuation as the person moves to the edge of the antenna beamwidths. The signal power is almost constant when the motion does not influence the standing waves.

Poisson process is the best distribution to describe burst arrivals. Thus, Poisson model was used to simulate the arrival time of a fade burst, i.e. $A T_{i} \sim \operatorname{Poisson}(\lambda \tau)$ 
where $\lambda$ is the Poisson rate, and $\tau$ is the fade interval. The fade rate used in this simulation is the average fade rate found from measurements.

Once a burst of fade arrives, the fade duration is simulated based on uniform distribution, i.e. $F D_{i} \sim U\left(F D_{\min }, F D_{\max }\right)$ where $F D_{\min }, F D_{\max }$ define the random variable interval and they were found also from measurements in which the minimum and maximum fade duration was obtained from identified-fading intervals and used to define the uniform distribution $F D_{\min }$ and $F D_{\max }$. Furthermore, to emulate the same fading shape resulted from measurements as described above, an attenuation factor was multiplied by the the first $n$ samples and last $n$ samples so as to get shallowdeep-shallow fade characteristic as those obtained from measurements. Figure B.2 shows a simulated bursty fading envelope where it can be seen all the aforementioned characteristics. 


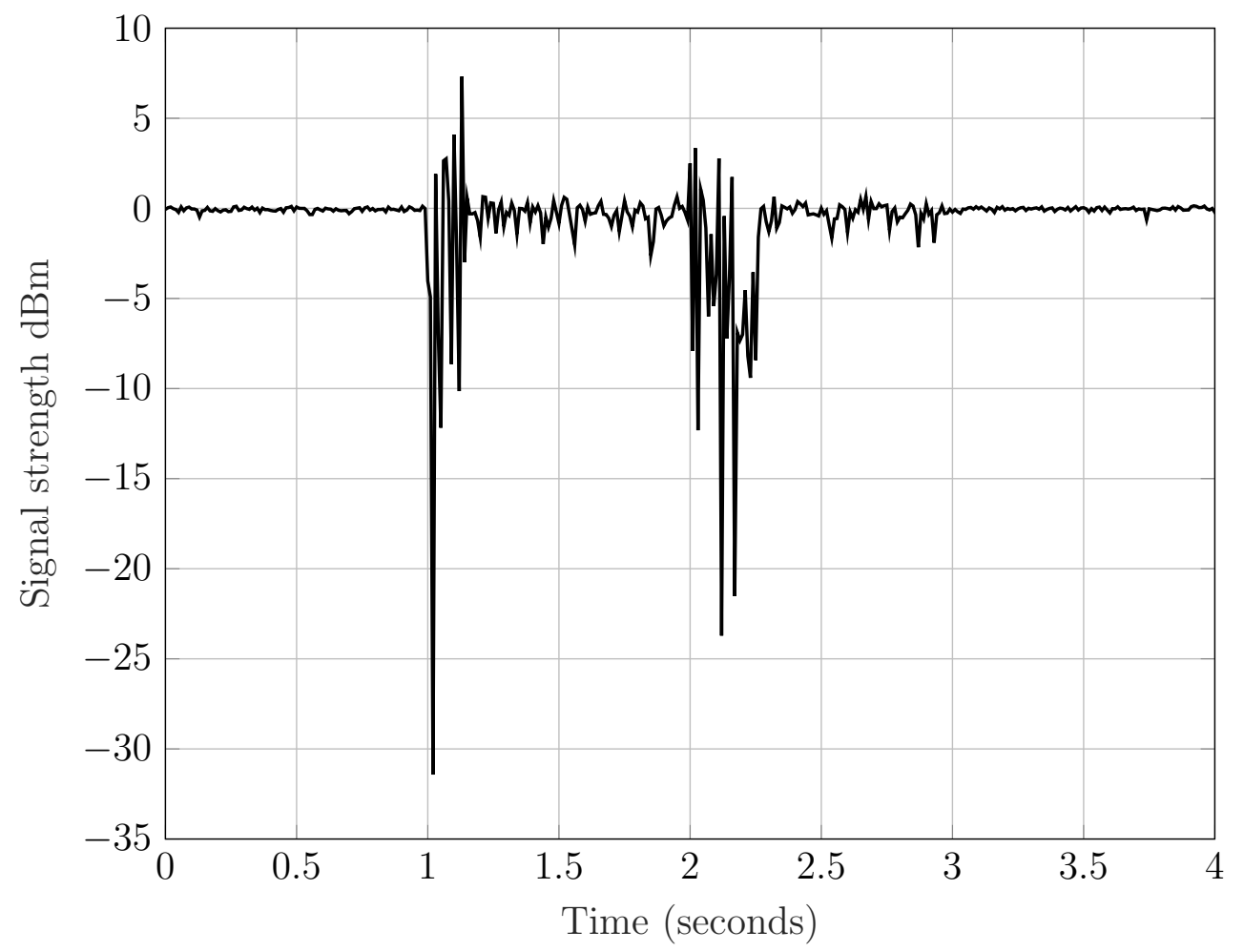

Figure B.2: Simulated bursty Rayleigh envelop fading 